\title{
The design and experimental studies of athermal silicon subwavelength grating waveguides
}

by

Marc Ibrahim, B.Eng.

A thesis submitted to the Faculty of Graduate and Postdoctoral Affairs in partial fulfillment of the requirements for the degree of

Master's of Applied Science

in

Electrical and Computer Engineering

Carleton University, Department of Electronics

Ottawa, Ontario, Canada

(C) 2013, Marc Ibrahim 
Library and Archives

Canada

Published Heritage

Branch

395 Wellington Street

Ottawa ON K1A ON4

Canada
Bibliothèque et

Archives Canada

Direction du

Patrimoine de l'édition

395 , rue Wellington

Ottawa ON K1A ON4

Canada
Your file Votre référence

ISBN: 978-0-494-94261-1

Our file Notre référence

ISBN: 978-0-494-94261-1
NOTICE:

The author has granted a nonexclusive license allowing Library and Archives Canada to reproduce, publish, archive, preserve, conserve, communicate to the public by telecommunication or on the Internet, loan, distrbute and sell theses worldwide, for commercial or noncommercial purposes, in microform, paper, electronic and/or any other formats.

The author retains copyright ownership and moral rights in this thesis. Neither the thesis nor substantial extracts from it may be printed or otherwise reproduced without the author's permission.
AVIS:

L'auteur a accordé une licence non exclusive permettant à la Bibliothèque et Archives Canada de reproduire, publier, archiver, sauvegarder, conserver, transmettre au public par télécommunication ou par l'Internet, prêter, distribuer et vendre des thèses partout dans le monde, à des fins commerciales ou autres, sur support microforme, papier, électronique et/ou autres formats.

L'auteur conserve la propriété du droit d'auteur et des droits moraux qui protege cette thèse. $\mathrm{Ni}$ la thèse ni des extraits substantiels de celle-ci ne doivent être imprimés ou autrement reproduits sans son autorisation.
In compliance with the Canadian Privacy Act some supporting forms may have been removed from this thesis.

While these forms may be included in the document page count, their removal does not represent any loss of content from the thesis.
Conformément à la loi canadienne sur la protection de la vie privée, quelques formulaires secondaires ont été enlevés de cette thèse.

Bien que ces formulaires aient inclus dans la pagination, il n'y aura aucun contenu manquant. 


\begin{abstract}
By conducting research on athermal silicon waveguides, viable CMOS compatible optoelectronic and photonic devices can be developed as temperature changes no longer affect the device performance. In such applications, appropriate waveguide dimensions, as well as materials with specific properties are required. Recently it was demonstrated that the subwavelength grating (SWG) effect gives an extra degree of freedom in the design of photonic circuits. The effective refractive index, in that case, is engineered through the use of waveguide core segments of different dielectric materials.
\end{abstract}

Here, we investigate athermal subwavelength grating waveguides. SWG structures with periodicities smaller than half the wavelength of light in the medium do not exhibit diffraction, reduces scattering, and can instead act as regular photonic waveguides of homogeneous effective core medium. Here we demonstrate both numerically and experimentally that by combining two materials with opposite thermo-optic coefficients, temperature independent behavior can be achieved. 


\section{Acknowledgements}

I would like to acknowledge the support of the staff of the Department of Electronics (DOE) at Carleton University who gave me access to a suitable and comfortable workplace. I am also grateful to the staff at the National Research Council (NRC) of Canada in Ottawa who let me work for them and gave me access to their facility for my research. I would like to acknowledge also the people I worked with at the facility: Dr. Jens H. Schmid, Dr. Pavel Cheben, Dr. Siegfried Janz, and Dr. Dan-Xia Xu. Their knowledge and experience have helped me develop my critical thinking and communication skills. I would like to thank my colleagues and fellow students, Yule Xiong, Vahidin Jupic, and Alireza Aleali who have answered my questions and helped me grow as a person and researcher. Lastly, I would like to thank my professor, advisor, and mentor Dr. Winnie N. Ye. Without her, I would have never been able to complete this dissertation. Your work, dedication, and input were inspiring and have pushed me to success. 


\section{Table of Contents}

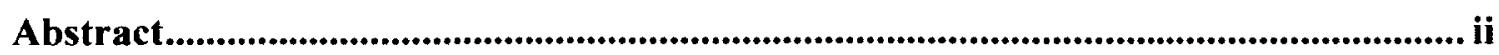

Acknowledgements .............................................................................................................ii

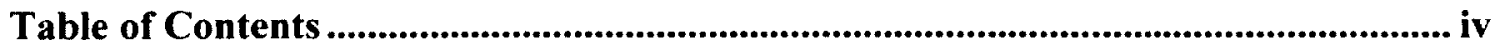

List of Tables ............................................................................................................................ vii

List of Illustrations................................................................................................................. viii

List of Appendices....................................................................................................................... $\mathbf{x}$

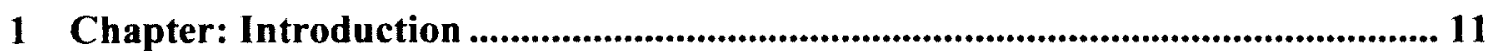

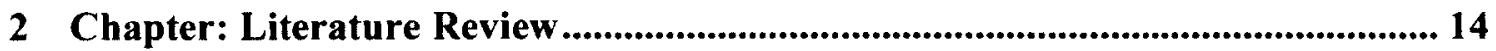

2.1 Past Work on the Design and Fabrication of Athermal Silica Waveguides ................. 14

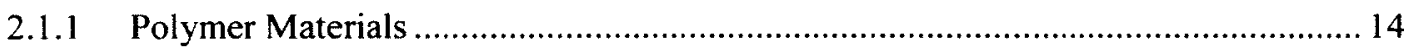

2.1.2 Mechanical Compensation and the Piezo-Optic Effect...................................... 17



2.2 Past Work on the Design and Fabrication of Athermal Silicon Waveguides ............... 19

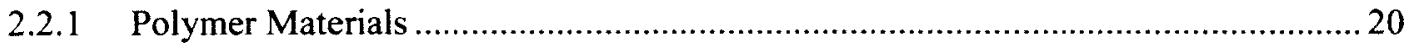

2.3 The Design and Fabrication of All-Polymer Athermal Waveguides........................... 21

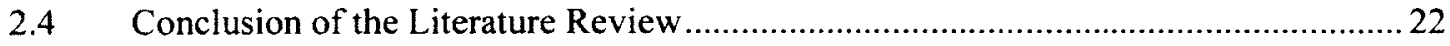

3 Chapter: Theory and Concepts ............................................................................. 23

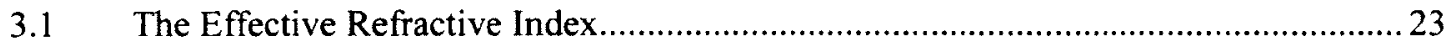

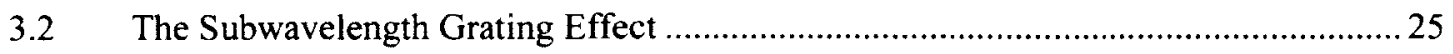

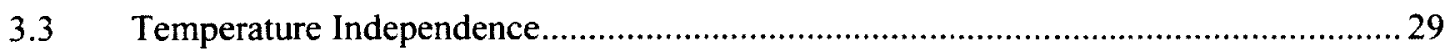

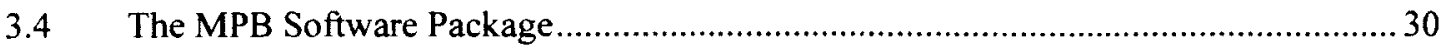

4 Chapter: Theoretical design of SWG/BSWG waveguides ................................... 32

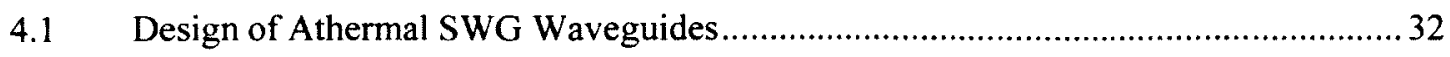




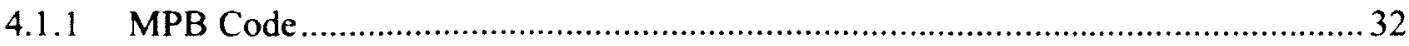

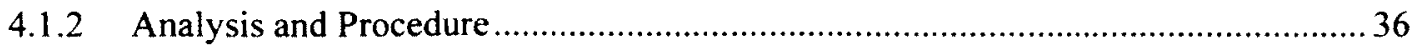



4.1.4 SWG Waveguide Tolerance and Thermal Bandwidth .........................................43

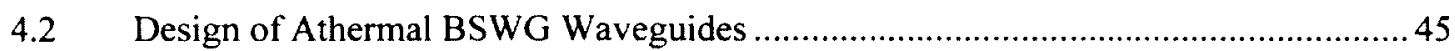

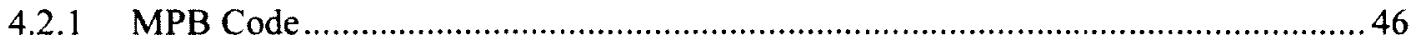

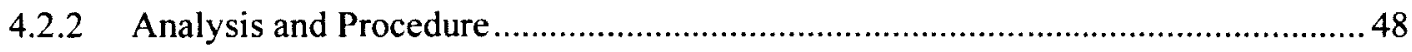

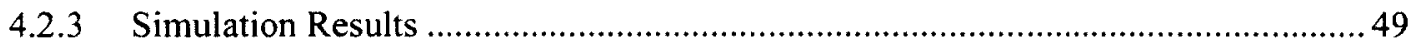

4.2.4 BSWG Waveguide Tolerance and Thermal Bandwidth ......................................52

5 Chapter: Demonstration of Athermal SWG/BSWG Waveguides...................... 55

5.1 The Fabrication and Realization of Athermal SWG Waveguides................................5 55

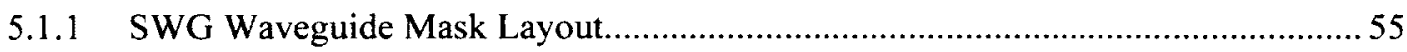

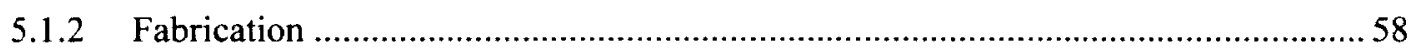

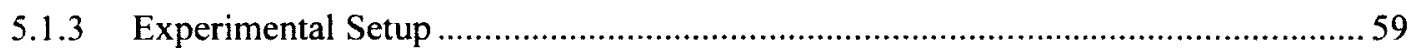

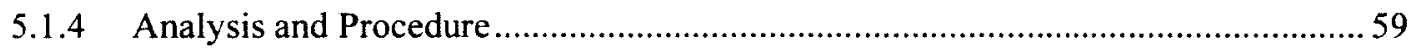

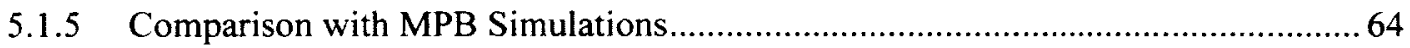

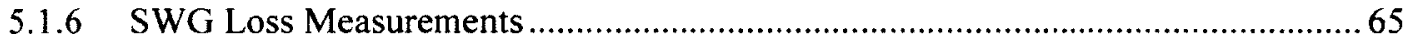

5.2 The Fabrication and Realization of Athermal BSWG Waveguides ............................68

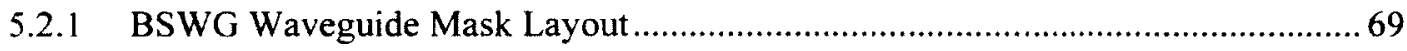

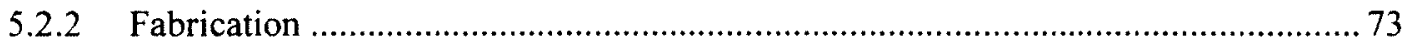

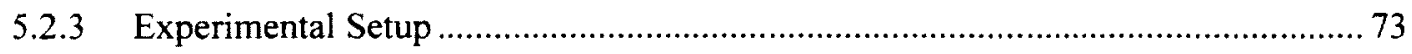

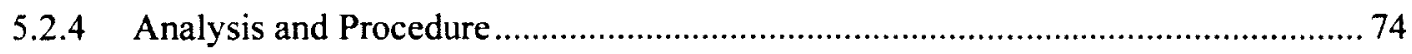

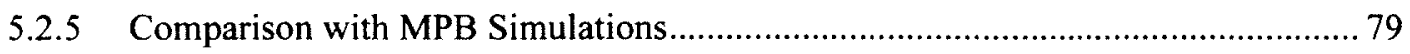

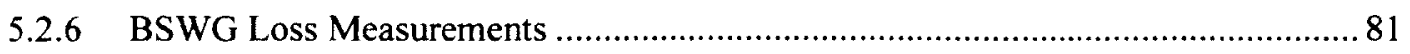

6 Chapter: Conclusion and Future Work..................................................................... 83

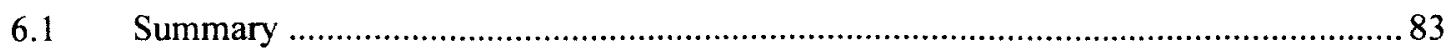


$6.2 \quad$ Future Work

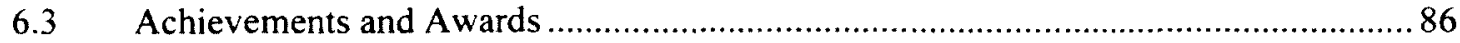

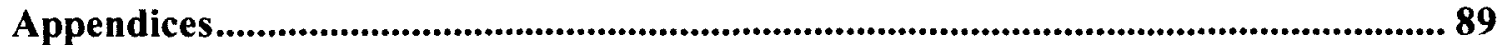

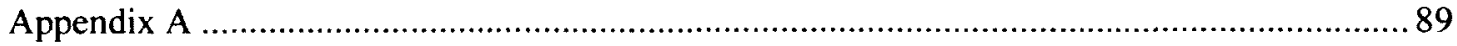

A.1 MPB Script and Terminology-SWG Waveguide Structure ................................... 89

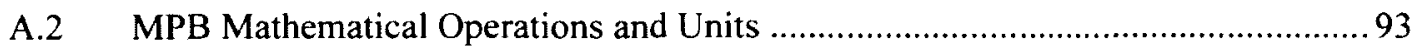





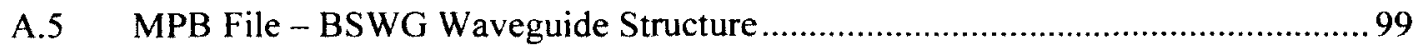



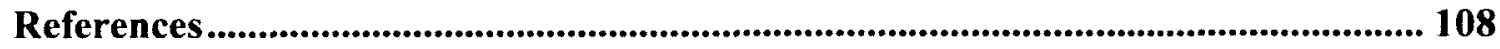




\section{List of Tables}

Table 4.1 The effective and group index dependence on the duty cycle .................... 41

Table 4.2 The calculated TO coefficient dependence on the duty cycle ...................... 42

Table 5.1 The propagation loss as a function of the SWG waveguide width (TE, TM) .. 67

Table 5.2 The propagation loss as a function of the BSWG waveguide width (TM)...... 82

Table 5.3 The propagation loss as a function of the BSWG bridge width (TM) ........... 82 


\section{List of Illustrations}

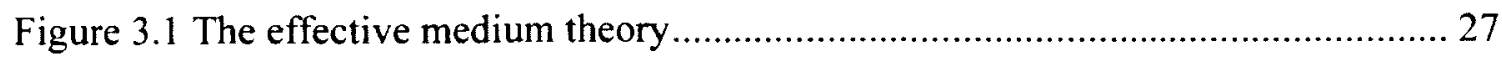

Figure 4.1 Schematic of a subwavelength grating waveguide core ............................ 33

Figure 4.2 SEM images of SWG waveguides .................................................. 34

Figure 4.3 The effective refractive index dependence on the temperature ..................... 35

Figure 4.4 The frequency dependence on the normalized wavevector (TE polarization) 36

Figure 4.5 Calculated waveguide TO coefficients as a function of the duty ratio........... 38

Figure 4.6 The athermal duty ratio dependence on the SWG waveguide width ............. 39

Figure 4.7 Calculated waveguide TO coefficients as a function of the duty ratio........... 39

Figure 4.8 The athermal duty ratio dependence on the SWG waveguide height ............40

Figure 4.9 The calculated TO coefficient dependence on the duty cycle ..................... 42

Figure 4.10 The TO coefficient dependence on the SWG waveguide parameters .......... 44

Figure 4.11 Two-dimensional Schematic top view of the BSWG waveguide ................ 46

Figure 4.12 Two-dimensional schematic top view of the BSWG (unit cell)................. 47

Figure 4.13 The effective refractive index dependence on the temperature .................. 48

Figure 4.14 The BSWG waveguide TO coefficient dependence on the bridge width ..... 49

Figure 4.15 The TO coefficient dependence on the bridge width ............................. 50

Figure 4.16 The bridge width dependence on the duty ratio .................................... 51

Figure 4.17 The TO coefficient dependence on the BSWG waveguide parameters ........ 52

Figure 4.18 The TO coefficient dependence on the bridge width (TE and TM) ............ 53

Figure 5.1 Screenshot of the SWG mask layout .................................................. 56

Figure 5.2 Schematic of the unbalanced MZI device with SWG sections .................... 57

Figure 5.3 SEM images of PW-SWG waveguide couplers .................................. 57 
Figure 5.4 Schematic of two microphotonic waveguides .................................... 58

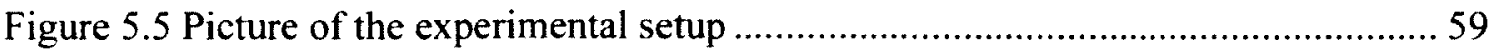

Figure 5.6 The dependence of the MZI transmittance on the wavelength.................... 62

Figure 5.7 The TO coefficient dependence on the wavelength (PW and SWG) ............63

Figure 5.8 The effective TO coefficient dependence on the SWG duty cycle ...............65

Figure 5.9 The output power dependence on the length of an SWG structure ...............66

Figure 5.10 The power dependence on the length of an SWG (with uncertainties) .........68

Figure 5.11 Screenshot of the BSWG mask layout (bottom and center) .................... 70

Figure 5.12 Screenshot of the top part of the BSWG mask layout ............................. 71

Figure 5.13 Close-up of different BSWG loss structures ......................................... 72

Figure 5.14 Sections of the PW-BSWG mode transformers .............................. 73

Figure 5.15 The spectral transmittance of the MZIs (PW and BSWG) ...................... 75

Figure 5.16 The TO coefficient dependence on the wavelength $(\mathrm{TM}, \mathrm{DC}=42 \%) \ldots \ldots . .76$

Figure 5.17 The TO coefficient dependence on the wavelength (other waveguides) ..... 78

Figure 5.18 The uncertainties in the BSWG waveguide parameters ............................ 79

Figure 5.19 The BSWG TO coefficient dependence on the bridge width ...................... 80 


\section{List of Appendices}

This page lists all of the appendices.

Appendix A - MPB Basics........................................... 89

A.1 MPB Script and Terminology-SWG Waveguide Structure..................89

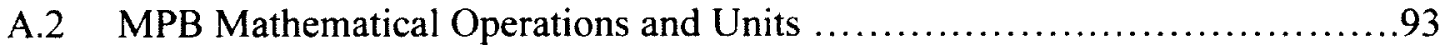

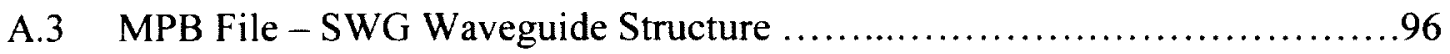

A.4 MPB Script - BSWG Waveguide Structure ..........................97

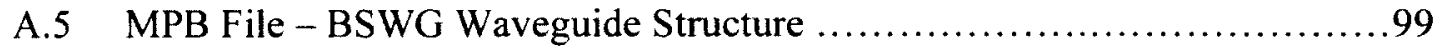

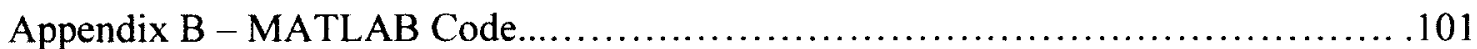




\section{Chapter: Introduction}

In recent years, research work in integrated silicon (Si) photonic devices and circuits has grown significantly. Such devices are expected to become important in optical data transport, spectroscopy, and biosensing applications [1]. Waveguide technology, in general, has become indispensable in people's lives. Everyone uses cell phones, the media, and the Internet to communicate and exchange information quickly and reliably. To transmit this information, optical waveguides are often utilized. In a telecommunications network for example, optical functions such as switching, routing, coupling, transport, and wavelength splitting are required. The implementation of directional couplers, microring resonators, Mach-Zehnder interferometers (MZIs), and arrayed waveguide gratings (AWGs) become necessary when performance is imperative. The use of silicon in this technology generates low costs in time and money accentuating the large amount of research work reported.

Yet, an important issue to consider in designing silicon devices is their temperature stability $[1,2]$. Changes in temperature have a large impact on materials with high thermo-optic (TO) coefficients $(\mathrm{dn} / \mathrm{dT})$ such as silicon (Si), resulting in unwanted perturbations. Devices such as the Mach-Zehnder interferometer (MZI) will not be optically stable in the presence of temperature fluctuations as any change in the refractive index will cause wavelength shifts of the optical output signals. Therefore, precise temperature control is often required. Alternatively, athermal silicon waveguides would be ideal for viable CMOS compatible optoelectronic and photonic devices as temperature 
changes would no longer affect the device performance. Thermal stability remains a significant issue in electronic-photonic integrated circuits because of the heterogeneous heating resulting from the integration with electronics [1]. The use of an external temperature control unit is an obstacle from the viewpoint of cost, device size, reliability, 3 practicality, and energy consumption [3].

Waveguides are considered to be "athermal" when their mode effective refractive index $\left(\mathrm{n}_{\mathrm{eff}}\right)$ does not vary with temperature $(\mathrm{T})$, i.e. $\mathrm{dn}_{\mathrm{eff}} / \mathrm{dT}=0$. The subsequent chapter discusses the previous work that has been done in regards to the design of athermal waveguides and devices comprising these, such as arrayed waveguide gratings (AWGs). A recent history of the work that has been published will be summarized there ordered according to the waveguide material systems and techniques that have reportedly been used. These techniques include the use of polymers, mechanical compensation, and others. The former technique of using polymers is the one we chose to pursue to fabricate our own athermal subwavelength grating (SWG) waveguides.

Chapter 3 covers the background knowledge that is necessary for the reader to understand the results of the experiment. Key concepts such as the propagation of light inside a medium, the subwavelength effect, the conditions for achieving temperature independence as well as the intentions of the MIT Photonics Bands (MPB) package, a useful tool for numerically solving Maxwell's equations are explained. Chapters 4 and 5, on the other hand, cover the theoretical and experimental results of the experiment whereas Chapter 6 is a concluding chapter summarizing the outcomes of the experiment. 
Further work and possible applications of temperature-independent SWG waveguide structures are discussed.

My contributions in this project include the theoretical analysis of subwavelength grating (SWG) waveguides as well as the theoretical and experimental analysis of bridged subwavelength grating (BSWG) waveguides, an alternate waveguide structure. The experimental studies of SWG waveguides were performed by Dr. J. H. Schmid, a researcher from the National Research Council (NRC) of Canada. 


\section{Chapter: Literature Review}

Most materials are quite susceptible to temperature variations. Their optical properties such as their refractive index will vary accordingly. During the past 20 years, many researchers have worked on the design and fabrication of athermal waveguides. Most of the listed work below has been on the design and fabrication of athermal silica waveguides. Yet, due to silicon's growth in the field of optics, there has been recent reported work on the design and fabrication of athermal silicon waveguides. Our project thus consists of using the advantages of subwavelength grating (SWG) structures to design and fabricate athermal silicon waveguides, not silica. This chapter summarizes the work on all temperature-independent waveguides done in the past.

\subsection{Past Work on the Design and Fabrication of Athermal Silica Waveguides}

\subsubsection{Polymer Materials}

In most cases, materials have a positive thermo-optic (TO) coefficient, but polymers, on the contrary, have a negative TO coefficient. They can hence be used to compensate for silicon's TO coefficient to significantly reduce the variations of the refractive index due to temperature.

First of all, Y. Kokubun et al. wrote several articles on the use of silica-based athermal waveguides with polymer cladding for temperature-independent lightwave devices such as narrowband optical filters and wavelength division multiplexing (WDM) systems 
$[3,4,5,6]$. A temperature independent narrow-band filter was realized by his group for the first time in 1996 as they fabricated a ring resonator operating at a wavelength of $1.3 \mu \mathrm{m}$ [7]. Two years later, in 1998 , his group realized another optical filter that operated at a wavelength of $1.55 \mu \mathrm{m}$ instead [8]. Their optical path length was independent of temperature.

In 1998, a temperature-independent Bragg grating within a ridge optical silica waveguide was designed for the first time by $D$. Bosc et al. by overlaying the structure with a polymer so that its temperature sensitivity could be reduced to a value of $0.003 \mathrm{~nm} /{ }^{\circ} \mathrm{C}$ or maybe even less [9].

In the span of 12 years, from 1997 to 2009 , much work on the design of silica-based temperature-independent AWGs was carried out. T. Saida, A. Takagi, S. Kamei, Y. Inoue, A. Kaneko and their respective groups first proposed a method for fabricating an athermal arrayed waveguide grating (AWG) containing low-loss grooves $[10,11,12]$ with optical add/drop multiplexers and double gate thermo-optical switches [13, 14]. The grooves were filled with silicone adhesive. The AWG multi/demultiplexer was later on synchronized with the MZI to create an athermal Mach-Zehnder interferometer (MZI) arrayed waveguide grating multi/demultiplexer [15, 16]. Furthermore, K. Maru et al. had successfully demonstrated an arrayed-waveguide grating (AWG) that is both athermal and center wavelength adjustable [17]. Athermal behavior was again achieved by filling the trenches with a polymer to compensate for the temperature-dependent wavelength shift. His group then fabricated a small and low-loss athermal AWG based on super-high- 
$\Delta$ waveguides with resin-filled trenches contained in the slab region [18]. K. Maru's group finally fabricated a compact low-loss athermal AWG module that is now based on $2.5 \%-\Delta$ silica-based waveguides [19]. A very small $2.5 \%-\Delta$ silica-based $100 \mathrm{GHz} 40$ channel athermal AWG module was also successfully demonstrated by $M$. Itoh's group [20]. Finally, a new compensation technique for suppressing second-order temperature dependence in athermal arrayed waveguide grating waveguides was proposed [21].

In 2003, T. Saito et al. developed a 32-channel $100 \mathrm{GHz}$ athermal silica-based AWG [22]. The AWG exhibited very small shifts of the center wavelength (less than $0.01 \mathrm{~nm}$ under a $70^{\circ} \mathrm{C}$ range). It was found that the athermal principle here is also applicable for AWGs with more than 40 channels. In the year, an athermal wavelength division multiplexing (WDM) stable single mode laser was proposed by G. Huang et al. [23]. The ridge waveguide Fabry-perot laser was modified by inserting a polymer grating structure for athermal single frequency operation.

In 2007 , De-Lu Li et al. thoroughly studied by numerical simulations the use of silica and polymer hybrid materials in the optimum design of temperature-independent AWGs [24]. It was found that the athermalization of the AWG can be realized by selecting the proper materials and structural dimensions of the waveguide. The device was fabricated on a silicon substrate.

In 2009 , K. Iwamoto et al. proposed an athermal optical waveguide design for silicabased planar lightwave circuits (PLCs) [25] based on K. Maru's technology [17, 18, 19]. 
The PLC circuit comprised low-refractive index polymer-filled trenches. The TO coefficients of the low-refractive index material and the waveguide core had opposite signs such that the temperature dependence of the device is suppressed. In addition, an athermal differential quadrature phase shift keying (DQPSK) demodulator was demonstrated the following year by Y. Nasu et al. [26]. The DQPSK demodulator was fabricated using K. Iwamoto's technology, i.e. the silica-based planar lightwave circuit (PLC) technology.

\subsubsection{Mechanical Compensation and the Piezo-Optic Effect}

An alternative technique for realizing athermal waveguides using mechanical compensation has been investigated throughout the past decade. In 2000 for example, N. Ooba et al. applied strain to a waveguide by using a bimetal plate [27]. The thermo-optic effect of the silica glass was compensated for by its strain-optic effect, reducing the wavelength shift to a value that is smaller than $0.05 \mathrm{~nm}$ per degree Celsius.

J. Hasegawa et al. developed silica-based AWG athermal modules with reported low loss and good reliability $[28,29]$ using a compensating copper plate. One of them operated at $200 \mathrm{GHz}$ containing 16 channels. The other operated at $100 \mathrm{GHz}$ containing 48 channels.

In 2007, further investigations on the temperature sensitivity of the silica-based AWG central wavelength were carried out by H. Huang et al. [30]. In their paper, they demonstrated that temperature sensitivity can be controlled using stress plates with different thermal expansion coefficients through which athermal behaviour can be 
achieved by choosing the proper substrate material with the correct negative thermal expansion coefficient. Furthermore, in that same year, an athermal silica-on-silicon AWG multiplexer/demultiplexer for E/C-band wavelength division multiplexing passive optical network (WDM-PON) application was proposed by H. K. Tae et al. [31]. The AWG's center wavelength was mechanically compensated by the adjustment of the input waveguide's position and aligned by a practical packaging method.

\subsubsection{Other Methods}

More recently, other novel techniques for designing temperature-independent glasses or waveguides have been proposed by different research groups. Photosensitive athermal $\mathrm{Ge}_{-} \mathrm{SiO}_{2}$ thin glass films were realized for the first time in 2003 by J. Nishii [32]. The Ge$\mathrm{SiO}_{2}$ thin glass films were then used for optical channel waveguide applications.

H. Hirota et al., in 2005, fabricated an athermal AWG that was composed of highly doped $\mathrm{TiO}_{2}$ and $\mathrm{SiO}_{2}$ [33]. The AWG was manufactured on a silicon substrate. The effective TO coefficient depended on the concentration of $\mathrm{TiO}_{2}$. Temperatureindependent behavior could therefore achieved by careful adjustment of the $\mathrm{TiO}_{2}$ concentration.

In 2008 , the design of a temperature-insensitive silica-based long-period waveguide grating was presented by B. D. Choudhury et al [34]. Control of the temperature sensitivity of the device was achieved here by varying the structural parameters of the waveguide, i.e the core and cladding thickness. The purpose of the design was to achieve 
an athermal condition for sensing the refractive index of an external medium. It is a good application example for demonstrating why athermal waveguides are often desired.

The following year, temperature stable operation of a silica-based AWG was achieved by filling the grooves of the grating with resin [35]. The technique that was used here consists of compensating the second-order temperature dependence of the passband wavelength by controlling the pressure-induced refractive index of the inserted resinfilled groove. Unwanted effects due to temperature change can be controlled by fine tuning of the refractive index. This work was published by $\mathrm{H}$. Terui et al.

In 2010, another novel method for manufacturing an athermal silica-based AWG was proposed by T. Zhou and W. Ma [36]. The method consists of applying UV adhesive or sticky gel in the gaps of the AWG by capillary infiltration. The spectra over a wide range of temperatures $\left(-40^{\circ} \mathrm{C}\right.$ to $\left.85^{\circ} \mathrm{C}\right)$ were almost identical hence demonstrating athermal behavior.

Finally, an athermal silica-based metal-free planar waveguide concave grating demultiplexer was proposed for the first time last year by C.-T. Lin [37]. A dielectric mirror at the grating facet was designed instead of using a metal coating on the back wall. To reduce the temperature sensitivity of the device, a three-focal-point method was introduced. Further explanations on the three-focal-point method are given in [37].

\subsection{Past Work on the Design and Fabrication of Athermal Silicon Waveguides}


More recently, much work on the temperature sensitivity of silicon-on-insulator (SOI) waveguides was also undertaken. In this section, we will briefly mention the history regarding the athermalization of the silicon waveguides. The use of polymers remains the fundamental method for achieving the athermal condition.

\subsubsection{Polymer Materials}

In 2008, X. Wang et al. proposed an athermal silicon arrayed waveguide grating (AWG) slot structure $[38,39]$. The temperature dependence of the effective index of the slot waveguides was carefully controlled by filling the slots with polymer material. Moreover, a more general approach in designing silicon-based polymer-cladded athermal highindex-contrast waveguides was presented by W. N. Ye, J. Michel, L. C. Kimerling, and L. Eldada [40]. More specifically, the design criteria and performance of silicon ring resonators for passive athermal applications were reported by the same people amongst others two years later [41]. The designs of temperature-independent high-index-contrast silicon waveguides can be different, but depend on the positive-negative TO composite structures, resonant wavelength dependent geometry, and the observation of small residual second order effects.

The following year, W. N. Ye's method for designing temperature-independent SOI waveguides was used for making temperature-independent silicon ring resonators. The rings were fabricated by J. Teng's group [42, 43]. The ring resonators were overlayed with polymer cladding placed on top of the silicon wires. In addition, L. Zhou et al. have further studied the design of athermal slotted silicon waveguides [44] and made an 
interesting discovery. They successfully showed that in a microring resonator structure, both filled (polymethyl methacrylate, PMMA) and unfilled (air-filled) overcladding slotted waveguides can achieve temperature-independent characteristics.

In 2010, Milan M. Milosevic et al. wrote an interesting article on the design of both athermal and low-loss ridge (rib) silicon waveguides [45]. They proved that for certain waveguide widths, low propagation losses for both TE (transverse electric) and TM (transverse magnetic) polarized light in silicon-based ridge waveguides are possible to achieve. They fabricated racetrack ring resonator structures which were covered by polymer material in order to aim for an athermal design resulting in a very small temperature dependent wavelength shift.

At last, in 2011, a temperature-independent SOI AWG was demonstrated for the first

time by L. Wang et al. by using a compensating cladding [46]. Since silicon's thermooptic (TO) coefficient is far more positive than silica's TO coefficient, a polymer whose TO coefficient is sufficiently large and negative had to be used. PSQ-LH polymer was chosen here.

\subsection{The Design and Fabrication of All-Polymer Athermal Waveguides}

In 2001 and 2002, an all-polymer athermal polarization-independent arrayed-waveguide grating multiplexer was manufactured and demonstrated for the first time $[47,48]$. The waveguides exhibited very good performances. The AWG comprised polymer waveguides placed on top of a polymer substrate. Temperature and polarization- 
independent behaviour were achieved here by properly adjusting the thermal expansion of the polymer substrate. Years after, in 2009, X. Han and his group were able to design an all-polymer temperature-independent waveguide microring resonator using the same technique [49].

\subsection{Conclusion of the Literature Review}

In conclusion, much work on temperature stability in waveguides has been reported in the last twenty years. Diverse methods such as the use of an external unit to control temperature, polymer claddings, resin, and bimetal plates to compensate using the strainoptic effect have been utilized in order to achieve athermal waveguide behavior. For this project, Prof. W. N. Ye's method for reaching athermalization was followed. The SOI waveguide was overlayed with an SU-8 polymer cladding. In our work, we have made use of the subwavelength effect in our waveguides in order to gain more control in choosing the structural waveguide parameters. The subwavelength effect along with its purpose will be thoroughly explained in the next chapter (Chapter 3). 


\section{Chapter: Theory and Concepts}

This chapter covers the background knowledge that is necessary to understand the meaning of this project's results and analysis. Key terms, equations, and concepts are explained here. The advantages of incorporating a subwavelength grating (SWG) in waveguides are discussed. Previous work on the possible applications of the SWG effect is also mentioned.

\subsection{The Effective Refractive Index}

We know from Maxwell's equations that light propagating in a material will experience reflections at material boundaries and travel at different speeds [50]. For non-magnetic transparent materials, the speed at which light travels inside a medium is defined by the refractive index (n). In other words, the refractive index of a material is the ratio between the speed of light (c) and the speed at which the light travels inside that material $\left(\mathrm{v}_{\mathrm{p}}\right)$ :

$$
n=\frac{c}{v_{p}}
$$

In addition, according to [50], the phase velocity of an electromagnetic wave is defined as:

$$
v_{p}=\frac{w}{k}
$$


Where $\omega$ is the angular frequency $(\omega=2 \pi f)$, and $k$ is the propagation constant or wave number. The propagation constant is defined as:

$$
k=\omega \sqrt{10}
$$

Where $\mu$ is the material's permeability, the ability of a material to support a magnetic field and $\varepsilon$ is the material's permittivity, the resistance encountered when forming an electric field. By combining equations (3.1), (3.2), and (3.3), we get an interesting relationship between the refractive index, the speed of light and the material's permittivity and permeability constants:

$$
n=c \sqrt{H E}=c \sqrt{\mu_{r} \mu_{0} Q_{r} B_{O}}
$$

Here, $\varepsilon_{\mathrm{r}}$ and $\mu_{\mathrm{r}}$ are respectively the relative permittivity and relative permeability whereas $\varepsilon_{0}$ and $\mu_{0}$ are the permittivity and permeability of free space. We must recall that the speed of light can also be defined in terms of the free space permeability and permittivity constants:

$$
c=\frac{1}{\sqrt{\mu_{0} \rho_{0}}}
$$


By combining equations (3.4) and (3.5), the relationship between the refractive index and the constants can be rewritten as:

$$
n=\sqrt{\theta_{r} \mu_{r}}
$$

For non-magnetic materials $\left(\mu=\mu_{0}, \mu_{r}=1\right)$, the refractive index is simply related to the material's relative permittivity, i.e. the material's dielectric constant:

$$
n=\sqrt{\theta_{r}} \quad(3.7)
$$

This relationship between the dielectric constant and the refractive index will be useful in calculating the effective thermo-optic (TO) coefficient of the SWG structures. The dielectric constants of the materials comprising the SWG and their temperature dependence are indeed important parameters (see Chapter 4 and Appendix A). We shall see how the effective refractive index of an SWG structure can be controlled in the subsequent section. For more information on the derivations of Maxwell's equations, phase velocity, group velocity, permeability, and permittivity, please refer to [50].

\subsection{The Subwavelength Grating Effect}

Conventional silicon planar waveguides consisting of a silicon core and silica cladding confine the light by index guiding within a core of higher refractive index than the cladding material [51]. They provide high confinement due to their high refractive index 
contrast. Silicon photonic wire waveguides are single mode channel waveguides of submicron dimensions, which can support bends with radii of only a few micrometers, thus making miniaturization of waveguide circuits possible. They have become a basic building block of silicon photonic device design. Some of the problems of photonic wire waveguides are the relatively large scattering loss caused by the large index contrast, the typically large overlap of the waveguide mode with the imperfections at the etched sidewalls of the waveguide structure and the difficulty of coupling light from an external optical fiber to the much smaller wire waveguide $[51,52,53]$. It has been shown that refractive index engineering with the subwavelength grating effect can help overcome some of these problems associated with the high refractive index contrast of the silicon/silica waveguide material system [54].

The subwavelength grating (SWG) effect essentially uses effective medium theory at the subwavelength regime where diffraction effects are suppressed (except for the $0^{\text {th }}$ order of diffraction) due to the size of the grating pitch that is embedded inside the waveguide $[51,54]$. Light here is confined in a SWG waveguide core, a composite material comprised of periodically alternating materials (silicon and silica glass, or polymers). For light incident normally to the grating, diffraction effects are suppressed when the grating period is smaller than the wavelength of the incoming light $(\lambda)$ according to the grating equation:

$$
\sin \theta=\frac{m A}{A}
$$


Where $\theta$ is the angle of diffraction, and $m$ is the order. The wavelength resonances resulting from diffraction do not exist when $\Lambda<\lambda$ [51]. According to photonic band structure theory, for light propagating along the periodicity of the grating, diffraction in the backward direction will occur if $\Lambda>\lambda / 2$ [55]. Light will propagate through the SWG structure without loss or diffraction if $\Lambda<\lambda / 2$. Moreover, when light is propagating through an SWG structure, it senses the average optical properties of the SWG medium, and therefore the materials comprising the SWG can be represented as a homogeneous effective medium with effective optical properties determined by the grating geometry. The SWG structure is essentially optically equivalent to a conventional photonic wire waveguide with a core material that is the combination of the comprising materials, i.e. the effective medium. Figure 3.1 illustrates that principle.
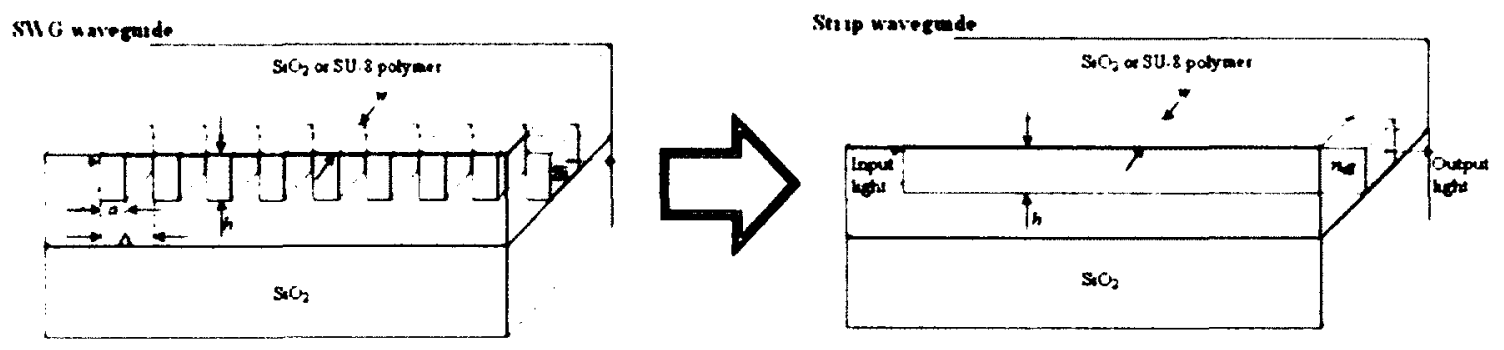

Figure 3.1 The effective medium theory

The SWG waveguide illustrated here (silicon and $\mathrm{SiO} 2 / \mathrm{SU}-8$ ) is represented as a photonic wire (PW) waveguide with an effective core medium with an effective index, $\mathrm{n}_{\mathrm{eff}}$. The effective index of the effective medium will vary as a function of the grating duty cycle $(a / \Lambda)$ or the filling factor, i.e. the fraction of material with silicon's index. Therefore, by increasing the size of the silicon segments, we also increase the value of $n_{e f f}$ 
Therefore, the SWG effect allows us to engineer artificial materials with intermediate effective indices ( $\mathrm{n}_{\mathrm{eff}}$ [56]. The resulting effective index will hence be determined by the comprising materials (core, cladding, substrate) and the grating geometry. For a one dimensional surface grating, the effective index is polarization dependent and is calculated in the following manner [56]:

$$
n_{\|}=\sqrt{f n_{1}^{2}+(1-f) n_{2}^{2}}
$$

Or

$$
n_{+}=\left[\frac{f}{n !}+\frac{\left(1-Q_{-}\right.}{n_{!}^{2}}\right]^{\frac{1}{3}}
$$

Where $n_{\|}$is the associated effective index when the electric field (E) of the incident light is parallel to the grating's grooves, $\mathrm{n} \perp$ is the associated effective index when the electric field is perpendicular to the grating's grooves, $\mathrm{n}_{1}$ and $\mathrm{n}_{2}$ are the refractive indices of the comprising media, and $\mathrm{f}$ is the filling factor. The filling factor is defined here as the fraction of material with index $n_{1}$. It is important to note that equations $3.9 \mathrm{a}$ and $3.9 \mathrm{~b}$ are only valid when the grating period is much smaller than the wavelength, i.e. $\Lambda / \lambda \rightarrow 0$. Further studies also show that the propagating mode in such structures is more delocalized, which can reduce large scattering losses at the boundaries [51].

Finally, after accurate adjustments of the dielectric constants, the effective index of the comprising SWG structure at any temperature and wavelength can also be calculated 
through equations (3.1) and (3.2) as the effective frequency dependence (f) on the wave number $(\mathrm{k})$ is given by the MIT Photonic Bands software described in section 3.4. The effective refractive index, in our context, can hence be interpreted as the ratio between the speed of light and the speed at which light effectively propagates in the SWG structure. For more clarifications on the basic ideas of the SWG effect, refer to [51-56].

\subsection{Temperature Independence}

As we have discussed in Chapter 2, several techniques for achieving temperature independence have been proposed by many researchers. In this project, we have opted for the utilization of a polymer (SU-8). In order to understand our procedure, we must first remember that the refractive index of a material changes as a function of temperature and is approximately linear around temperature $T=T_{0}$. The effective index within a temperature range around $T=T_{0}$ is calculated in the following manner:

$$
n(T)=n_{e}+\frac{d n}{d T} \Delta T
$$

Where $n_{0}$ is the refractive index of the material at temperature $T=T_{0}, d n / d T$ is the thermooptic (TO) coefficient of the material, and $\Delta T$ is the temperature difference between the actual temperature and the temperature at $T=T_{0}$. As we can see from (3.10), the variations of the effective index due to temperature change is determined by the value of the TO coefficient. Silicon, for example, has a relatively large TO coefficient of $1.9 \times 10^{-4} \mathrm{~K}^{-1}$. However, polymers such as SU-8 have negative TO coefficients [57]. In other words, 
their refractive index decreases as temperature increases. Hence, by combining SU-8 with silicon in a waveguide structure, achieving athermal behavior becomes possible as the SU-8 will counteract the contribution to the effective index of silicon's temperature induced refractive index change. In an SWG waveguide with a given periodicity, the volume fraction of SU-8 polymer is controlled by the size of the silicon core segments. For example, the effective TO coefficient of the SWG waveguide will end up being negative (polymer-like) if the segments are too small and positive (silicon-like) if they are too big. The aim of our designs is thus to identify the correct waveguide geometry to achieve athermal waveguide operation. We will either increase or decrease the structure's filling factor, i.e. the grating duty cycle (see chapters 4 and 5), until we achieve temperature independence.

\subsection{The MPB Software Package}

The MIT Photonic Bands (MPB) is a software package that will solve Maxwell's equations in periodic dielectric structures [58]. It was intended to study photonic crystals that exhibit a band gap in their respective optical modes where no light can propagate. The MPB software package can however be used for the study of the one dimensional subwavelength grating (SWG) and bridged subwavelength grating (BSWG). For more details on the BSWG, please consult Chapters 4 and 5. For more information on photonic crystals, please consult the book mentioned in [55].

The MPB software package studies periodic dielectric structures by approaching the problem in the frequency domain as oppose to many other software packages (e.g. FDTD 
packages) [58]. A big advantage of using such approach is that we get, from the software, both the frequencies, wave numbers, and the eigenstates at the same time. The eigenstates and eigenvalues of Maxwell's equations are directly computed and the electric and magnetic fields all have a respective definite frequency. We can thus look at the propagating modes immediately simplifying our analysis. A more detailed introduction of the MPB software package is given at the beginning of [58]. The basics of the MPB package are shown in Appendix A. Commands, mathematical operations, as well as the written scripts and output files are outlined. 


\section{Chapter: Theoretical design of SWG/BSWG waveguides}

This chapter covers the procedure for designing athermal silicon subwavelength (SWG) and bridged subwavelength grating (BSWG) waveguides. The numerical simulations and theoretical calculations required in order to find the thermo-optic (TO) coefficient are included. We also discuss their dependence of the simulation results on variations in waveguide height, width, and operating wavelength. The possible realization of temperature independent silicon BSWG waveguides for both TE and TM polarized is finally discussed.

\subsection{Design of Athermal SWG Waveguides}

The required steps for designing temperature independent SWG waveguides are reported here. The analysis for measuring the effective TO coefficient of diverse SWG structures from the MPB software is explained in detail. In chapter 5, the results will then be compared to the ones obtained experimentally.

\subsubsection{MPB Code}

Numerical simulations were carried out in order to calculate the TO coefficient for different waveguide configurations. The MPB software numerically solves Maxwell's equations for periodic structures, such as SWGs. Using this software, we were interested in calculating the waveguide's effective refractive index $\left(\mathrm{n}_{\mathrm{eff}}\right)$ at a certain temperature. Different parameters such as the waveguide physical dimensions and dielectric constants were used as input parameters for the calculations. We will investigate later how the 
results depend on these parameters. A written script describing a possible SWG waveguide structure is shown in Appendix A (see section A.1).

The MPB script shown in section A.1 describes an SWG structure with a periodicity (grating pitch) of $250 \mathrm{~nm}$, a height of $260 \mathrm{~nm}$, and a width of $470 \mathrm{~nm}$. The height and width here are defined as the thickness and the width of the silicon channel. The length of each segment is $200 \mathrm{~nm}$. The waveguide described in the script has thus a grating duty cycle of $80 \%$. A schematic of the SWG structure showing the relevant parameters is illustrated in Fig. 4.1. SEM images of different SWG waveguide configurations are shown in Fig. 4.2.

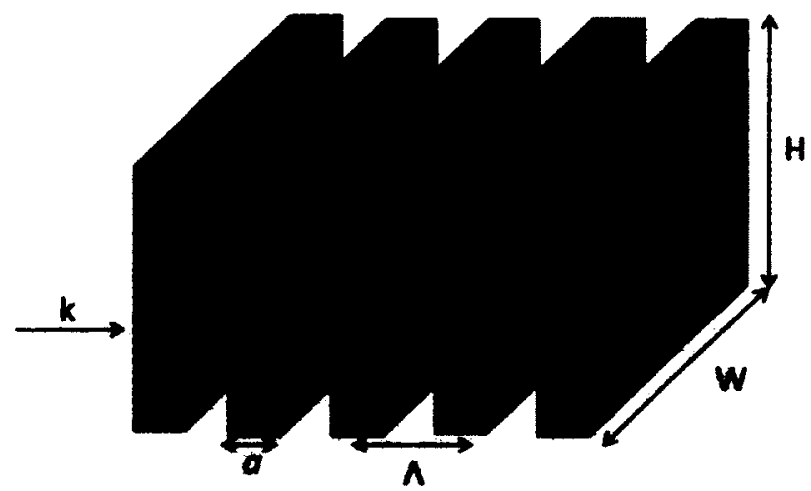

Figure 4.1 Schematic of a subwavelength grating waveguide core

$\mathrm{W}$ is the waveguide width, $\mathrm{H}$ is the waveguide height, $\mathrm{a}$ is the length of a silicon core segment, $\Lambda$ is the grating pitch (period), $\mathrm{k}$ is the wavevector, and $\mathrm{nl}$ and $\mathrm{n} 2$ are the core and cladding indices respectively. 


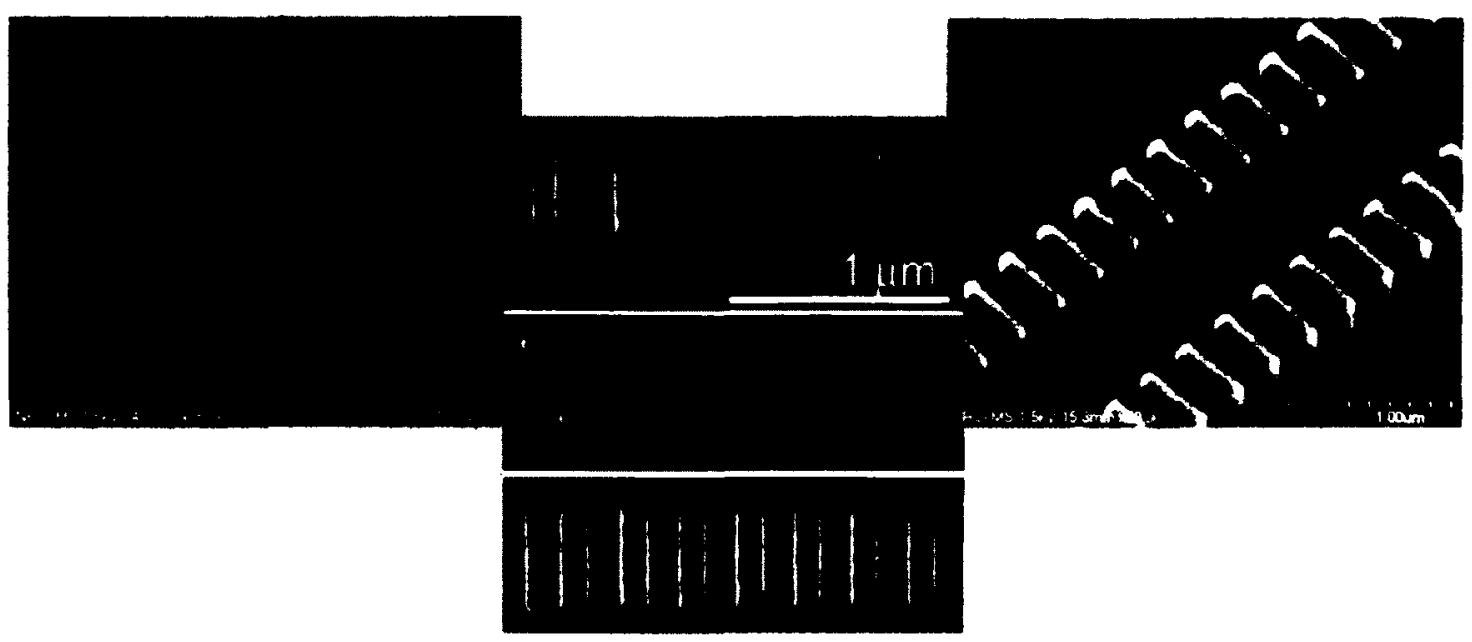

Figure 4.2 SEM images of SWG waveguides

These waveguides were designed to have nominal widths, heights, and periodicities of $450 \mathrm{~nm}, 260 \mathrm{~nm}$, and $250 \mathrm{~nm}$ respectively.

Other important input parameters of the script are the dielectric material constants. Since the relative permeability (see section 2.1) of the materials comprising the waveguide is $\mu_{\mathrm{r}}$ $=1$, the dielectric constants $\left(\varepsilon_{\mathrm{r}}\right)$ of silicon $(\mathrm{Si})$, SU-8 polymer, and the oxide are simply the square of their respective refractive index $\left(\varepsilon_{r}=n^{2}\right)$. The refractive index of certain materials, and consequently their dielectric constant, vary with temperature. To find the effective thermo-optic coefficient $\left(\mathrm{dn}_{\mathrm{eff}} \mathrm{dT}\right)$ of the waveguide, we use the dielectric constants of the materials comprising the waveguide structure at the different temperatures, say $T=273 \mathrm{~K}, T=293 \mathrm{~K}$, and $\mathrm{T}=313 \mathrm{~K}$ (see Fig. 4.3). The refractive indices of the materials at room temperature $(T=293 \mathrm{~K})$ are found in the literature [2]. The refractive index of silicon, $\mathrm{SU}-8$ and the oxide are respectively $\mathrm{n}_{\mathrm{Si}}=3.476, \mathrm{n}_{\mathrm{SU}-8}=$ 1.58 , and $\mathrm{n}_{\mathrm{SiO} 2}=1.444$. To reflect a temperature increase and decrease of $20 \mathrm{~K}$ for the purpose of measuring the effective TO coefficient of the SWG structure, the refractive 
indices of the materials are changed from their room-temperature according to their material TO coefficient value:

$$
n(T)=n_{0}+\frac{d n}{d T} \Delta T
$$

Where $n_{0}$ is the refractive index of the material at room temperature $(T=293 K)$, and $\Delta T$ is the temperature difference between the sample and room temperatures. The TO coefficients $(\mathrm{dn} / \mathrm{dT})$ of silicon, SU-8, and silicon dioxide are $1.9 \times 10^{-4} \mathrm{~K}^{-1},-1.1 \times 10^{-4} \mathrm{~K}^{-1}$, and $9.33 \times 10^{-6} \mathrm{~K}^{-1}$, respectively. Since the TO coefficient of the buried oxide layer is much smaller than Si and SU-8, its effect here is assumed to be negligible [59].

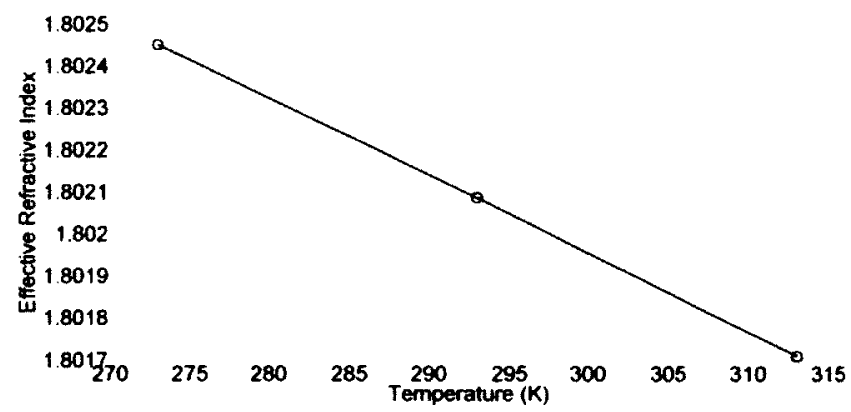

Figure 4.3 The effective refractive index dependence on the temperature

The waveguide width, height, grating pitch, and grating duty cycle are $450 \mathrm{~nm}, 260 \mathrm{~nm}, 300 \mathrm{~nm}$, and $50 \%$ respectively. Light is TE polarized with a wavelength of $1550 \mathrm{~nm}$.

Further explanations on how to use the MPB generated file obtained from a simulation trial are explained in Appendix A. An example file is shown in section A.2. The MPB file 
shown in the appendix contains the telecommunications frequencies (around $f=193.55$ $\mathrm{THz}$ ) of the TE fundamental mode that is propagating inside an SWG structure with $\mathrm{W}=$ $450 \mathrm{~nm}, \mathrm{H}=260 \mathrm{~nm}, \Lambda=250 \mathrm{~nm}$, and $\mathrm{DC}=62 \%$. Fig. 4.4 is a plot generated by the MPB software which illustrates the frequency dependence on the wavevector.

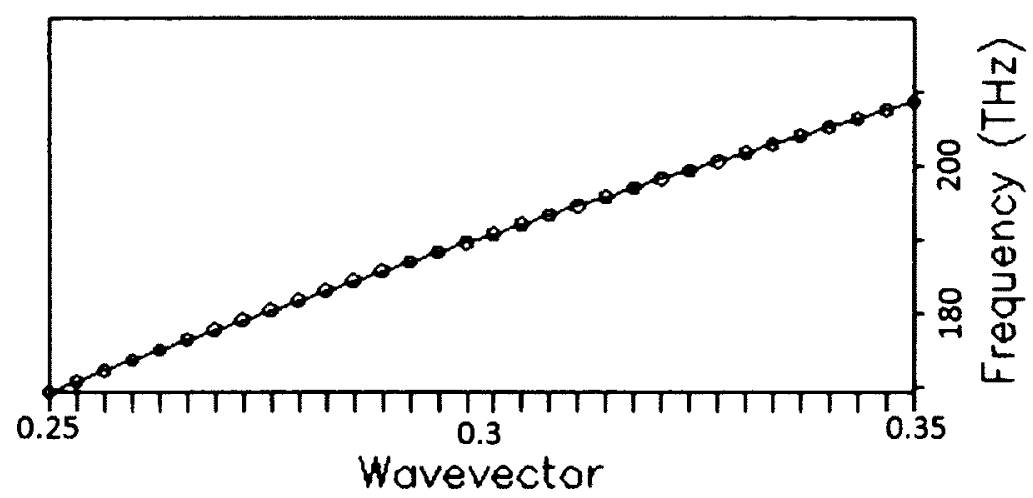

Figure 4.4 The frequency dependence on the normalized wavevector (TE polarization)

The frequency dependence here is evaluated at room temperature. The normalized wavevector range is from 0.25 to 0.35 and the frequency range is from $170 \mathrm{THz}$ to around $220 \mathrm{THz}$. The SWG waveguide width, height, grating pitch, and grating duty cycle are $450 \mathrm{~nm}, 260 \mathrm{~nm}, 250 \mathrm{~nm}$, and $62 \%$ respectively.

\subsubsection{Analysis and Procedure}

The first step in our procedure for designing SWG waveguides was to calculate the waveguide's effective refractive index ( $\left.n_{\mathrm{eff}}\right)$. The effective refractive index of the SWG waveguide at different temperatures near room temperature $(273 \mathrm{~K}, 293 \mathrm{~K}$, and $313 \mathrm{~K}$ ) was obtained from simulations of the structure where the bulk material refractive indices of silicon and SU-8 polymer are varied according to their respective material TO coefficients. These effective indices were then found from the MPB data files where the normalized frequencies $\left(f_{\text {norm }}\right)$ and respective wave numbers $\left(k_{\text {norm }}\right)$ are transcribed (see 
section 4.1.1). Since we are interested in designing an athermal waveguide at a wavelength of $1550 \mathrm{~nm}$, we have to look for and record the normalized k-value corresponding to a frequency of $193.55 \mathrm{THz}$. Further explanations on how to calculate the effective index from the normalized frequency $\left(f_{\text {norm }}\right)$ and wave number $\left(k_{\text {norm }}\right)$ are given in Appendix A.

The next step was to calculate the waveguide's TO coefficient $\left(\mathrm{dn}_{\mathrm{eff}} / \mathrm{dT}\right)$. Now that we have the waveguide's effective index at several temperatures, a simple plot illustrating the dependence of the effective index on the temperature is sufficient. The TO coefficient is obtained from the slope of the plot's regression (best fit) line. Figure 4.3 shown in section 4.1 .1 is an example plot.

The slope $(\mathrm{m})$ of this particular regression line is $\mathrm{m}=-1.849 \times 10^{-5} \mathrm{~K}^{-1}$. The TO coefficient was found for varying waveguide grating duty cycles until athermal behavior is achieved. The waveguide width, height and grating pitch were at first held constant. We then repeated the same steps, but with different waveguide configurations. Different athermal designs exist for various waveguide widths, heights, and periodicities as long as the latter remains in the subwavelength regime. The required duty cycle for athermal operation at $\lambda$ $=1550 \mathrm{~nm}$ is hence dependent on the polarization of light and waveguide geometrical parameters. 


\subsubsection{Simulation Results}

Fig. 4.5 a-b show the dependence of the effective TO coefficients $\left(\mathrm{dn}_{\mathrm{eff}} \mathrm{dT}\right)$ at a wavelength of $1550 \mathrm{~nm}$ to the duty ratio predicted by our simulations for different waveguide widths. The waveguides were set to a height and grating pitch of $260 \mathrm{~nm}$ and $250 \mathrm{~nm}$ respectively.

(a) TE Polarization

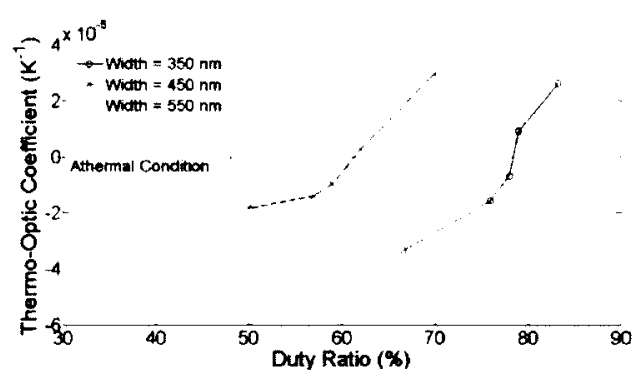

(b) TM Polarization



Figure 4.5 Calculated waveguide TO coefficients as a function of the duty ratio Calculated waveguide TO coefficients as a function of the duty ratio for various SWG waveguide widths

To be temperature independent, waveguides of $350 \mathrm{~nm}$ in width for example must have corresponding duty ratios of $79 \%$ and $90.2 \%$ for TE and TM polarizations, respectively. With increasing width, a smaller duty ratio is required for athermal behavior. In other words, a smaller fraction of silicon is needed to make up the waveguide core. In addition, for a given width, TE polarization requires a smaller duty cycle than TM. The athermal grating duty ratios also vary more strongly for TE polarized light, as illustrated in Fig. 4.6. This behavior is expected since the profile of the fundamental mode for TE polarized light is normally distributed along the width of the waveguide compared to the mode profile for TM light where the distribution is along the waveguide height. 


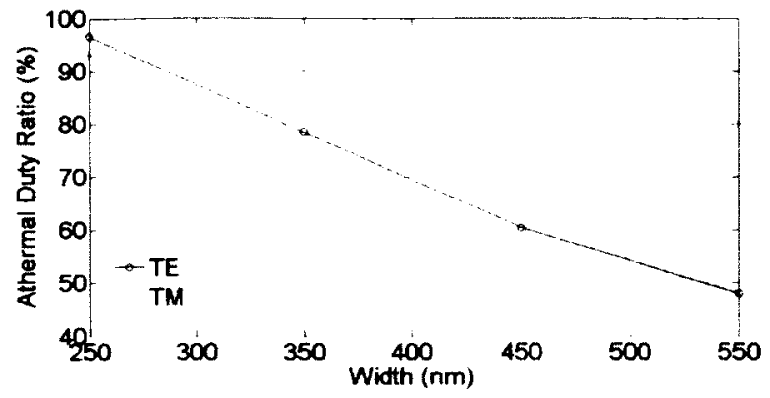

Figure 4.6 The athermal duty ratio dependence on the SWG waveguide width

Similarly, the effective waveguide TO coefficients $\left(\mathrm{dn}_{\mathrm{eff}} / \mathrm{dT}\right)$ as a function of the duty ratio for different waveguide heights were also calculated and are illustrated in Fig. 4.7 ab. The waveguides were set to a width and grating pitch of $450 \mathrm{~nm}$ and $300 \mathrm{~nm}$ respectively. As expected, grating duty ratios required for temperature independent behavior were also dependent on the height of the device.

(a) TE Polarization

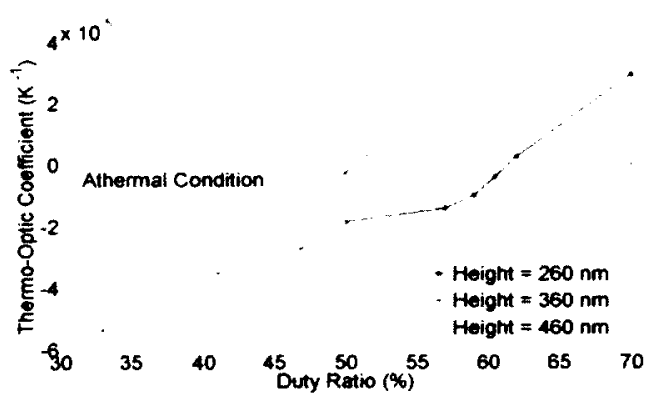

(b) TM Polarization

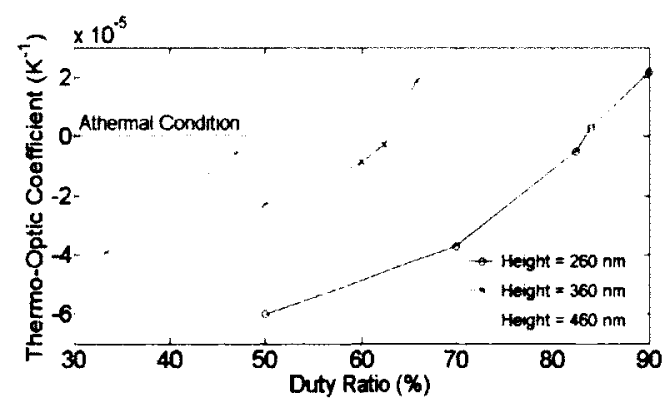

Figure 4.7 Calculated waveguide TO coefficients as a function of the duty ratio

Calculated waveguide TO coefficients as a function of the duty ratio for various SWG waveguide heights

In such cases, athermal waveguides of $260 \mathrm{~nm}$ in height for example must have grating duty ratios of $61.3 \%$ and $83.3 \%$ for $\mathrm{TE}$ and $\mathrm{TM}$ polarizations, respectively. With increasing height, a smaller duty ratio is required for athermal behavior. For a given 
height, TE polarization generally requires a smaller duty cycle than TM. Athermal grating duty ratios also vary more strongly for TM polarized light (see Fig. 4.8). This is the opposite of the behavior observed for varying waveguide width. Note that for a height of $460 \mathrm{~nm}$, the required athermal duty cycle for TE polarized light is actually slightly larger than the one for TM $(49.7 \%$ for TE polarized light, and $48 \%$ for TM polarized light). We can also notice that if the waveguide's height is equal to the waveguide's width ( $260 \mathrm{~nm}$ in the case of Fig.4.6, and $450 \mathrm{~nm}$ in the case of Fig. 4.8), the realization of a temperature-independent SWG waveguide design that works for both polarizations is achievable. In practice however, the choice of the waveguide size may be restricted by other factors. The height of our fabricated SWG waveguides, for example, was restricted to $260 \mathrm{~nm}$ due to the availability of SOI wafers for this work.

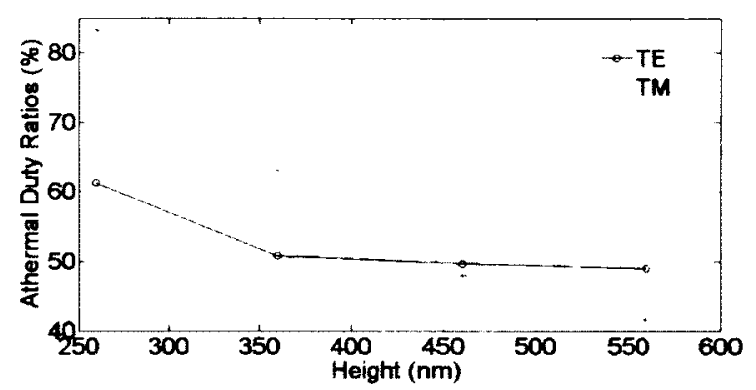

Figure 4.8 The athermal duty ratio dependence on the SWG waveguide height

Our fabricated SWG structures hadg a grating pitch of $250 \mathrm{~nm}$ and further simulations with the actual waveguide dimensions were consequently carried out. The SWG waveguides on the sample had a width of $450 \mathrm{~nm}$, and a height of $260 \mathrm{~nm}$. Table 4.2 and Fig. 4.9 depict the dependence of the TO coefficient on the duty ratio (duty cycle) of such a waveguide. The effective ( $\left.n_{\text {eff }}\right)$ and group $\left(n_{g}\right)$ indices at room temperature $(T=293 K)$ 
were also calculated over a whole range of duty cycles and are shown in table 4.1. These give us further insight on the possible reflections and coupling losses due to the refractive index mismatch.

\begin{tabular}{|c|c|c|c|c|c|}
\hline $\begin{array}{c}\text { Segment } \\
\text { Length }(\mathrm{nm})\end{array}$ & $\begin{array}{c}\text { Duty Cycle } \\
(\%)\end{array}$ & $\begin{array}{c}\text { Effective } \\
\text { Index }\left(\mathrm{n}_{\mathrm{en}}\right) \\
\text { (TE light) }\end{array}$ & $\begin{array}{c}\text { Group Index } \\
\left(n_{\mathrm{g}}\right) \\
\text { (TE light) } \\
\end{array}$ & $\begin{array}{c}\text { Effective } \\
\left.\text { Index ( } n_{\text {eff }}\right) \\
\text { (TM light) }\end{array}$ & $\begin{array}{c}\text { Group Index } \\
\left(n_{\mathrm{g}}\right) \\
(\mathrm{TM} \text { light })\end{array}$ \\
\hline 100 & 40 & 1.689 & 2.245 & 1.596 & 1.780 \\
\hline 125 & 50 & 1.802 & 2.778 & 1.631 & 1.999 \\
\hline 137.5 & 55 & 1.834 & 2.898 & $\square$ & 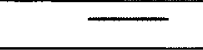 \\
\hline 150 & 60 & 1.875 & 3.043 & 1.657 & 2.155 \\
\hline 152.5 & 61 & 1.900 & 3.114 & $=$ & 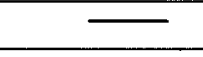 \\
\hline 155 & 62 & 1.912 & 3.159 & $\longrightarrow$ & $=$ \\
\hline 162.5 & 65 & 1.953 & 3.272 & $=$ & 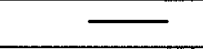 \\
\hline 175 & 70 & 2.003 & 3.385 & 1.709 & 2.464 \\
\hline 187.5 & 75 & 2.059 & 3.514 & 1.761 & 2.791 \\
\hline 200 & 80 & 2.154 & 3.691 & 1.793 & 2.965 \\
\hline 205 & 82 & $\square$ & $\square$ & 1.802 & 3.019 \\
\hline 210 & 84 & - & - & 1.833 & 3.181 \\
\hline 212.5 & 85 & - & 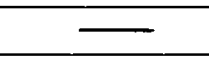 & 1.843 & 3.239 \\
\hline 225 & 90 & 2.293 & 3.877 & 1.906 & 3.545 \\
\hline 250 & 100 & 2.536 & 4.057 & 2.076 & 4.206 \\
\hline
\end{tabular}

Table 4.1 The effective and group index dependence on the duty cycle

The effective and group index dependence on the duty cycle of a waveguide with a width, height and grating pitch of $450 \mathrm{~nm}, 260 \mathrm{~nm}$ and $250 \mathrm{~nm}$, respectively 


\begin{tabular}{|c|c|c|c|}
\hline Segment Length (nm) & Duty Cycle (\%) & $\begin{array}{c}\text { TO Coefficient (K } \\
\text { (TE light) }\end{array}$ & $\begin{array}{c}\text { TO Coefficient }\left(\mathbf{K}^{-1}\right) \\
\text { (TM light) }\end{array}$ \\
\hline 100 & 40 & $-4.943 \mathrm{E}-05$ & $-6.952 \mathrm{E}-05$ \\
\hline 125 & 50 & $-1.870 \mathrm{E}-05$ & $-6.188 \mathrm{E}-05$ \\
\hline 137.5 & 55 & $-1.414 \mathrm{E}-05$ & - \\
\hline 150 & 60 & $-4.202 \mathrm{E}-06$ & $-5.690 \mathrm{E}-05$ \\
\hline 152.5 & 61 & $2.887 \mathrm{E}-06$ & - \\
\hline 155 & 62 & $6.038 \mathrm{E}-06$ & - \\
\hline 162.5 & 65 & $1.225 \mathrm{E}-05$ & $-4.281 \mathrm{E}-05$ \\
\hline 175 & 70 & $2.944 \mathrm{E}-05$ & $-2.670 \mathrm{E}-05$ \\
\hline 187.5 & 75 & $4.337 \mathrm{E}-05$ & $-1.654 \mathrm{E}-05$ \\
\hline 200 & 80 & $7.129 \mathrm{E}-05$ & $-1.288 \mathrm{E}-05$ \\
\hline 205 & 82 & - & $-1.972 \mathrm{E}-06$ \\
\hline 210 & 84 & - & $1.298 \mathrm{E}-06$ \\
\hline 212.5 & 85 & - & $2.641 \mathrm{E}-05$ \\
\hline 225 & 90 & $1.106 \mathrm{E}-04$ & $1.140 \mathrm{E}-04$ \\
\hline 250 & 100 & $1.825 \mathrm{E}-04$ & \\
\hline
\end{tabular}

Table 4.2 The calculated TO coefficient dependence on the duty cycle

The calculated TO coefficient dependence on the duty cycle of a waveguide with a width, height and grating pitch of $450 \mathrm{~nm}, 260 \mathrm{~nm}$ and $250 \mathrm{~nm}$, respectively

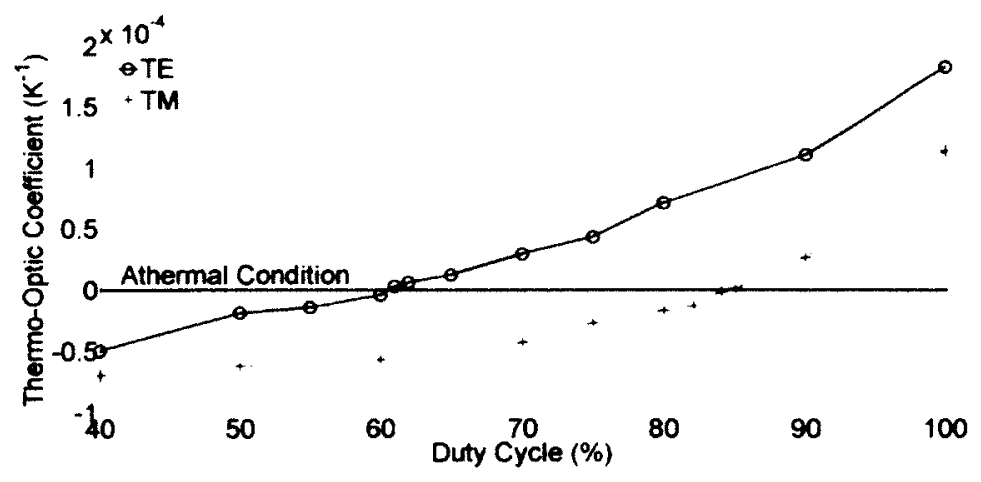

Figure 4.9 The calculated TO coefficient dependence on the duty cycle

The calculated TO coefficient dependence on the duty cycle of a waveguide with a width, height and grating pitch of $450 \mathrm{~nm}, 260 \mathrm{~nm}$ and $250 \mathrm{~nm}$, respectively

According to the calculations, to be temperature independent, these waveguides must have duty ratios of about $60.7 \%$ and $84.6 \%$ for TE and TM polarizations, respectively. 
The results are almost identical to the ones required for a waveguide width, height and grating pitch of $450 \mathrm{~nm}, 260 \mathrm{~nm}$ and $300 \mathrm{~nm}$ respectively. The latter structures needed a duty ratio of $61 \%$ and $82.5 \%$ for TE and TM polarized respectively in order to achieve athermal operation (see Fig. 4.7-4.9). Therefore, we conclude that changing the grating pitch in the design of athermal SWG waveguides does not greatly affect the results provided that the length of the core segments is correctly adjusted. The size of the pitch must however remain in the subwavelength regime restricting the use of much larger periodicities. Choosing small core segments, on the other hand, makes fabrication of the SWG waveguides more challenging.

\subsubsection{SWG Waveguide Tolerance and Thermal Bandwidth}

Investigations on the tolerances of the thermo-optic coefficient of nearly temperatureindependent SWG waveguides to variations in the other waveguide parameters were also carried out. Starting from our reference waveguide with a width of $450 \mathrm{~nm}$ and a height of $260 \mathrm{~nm}$, the effects due to small changes in the width, height, and operating wavelength were numerically calculated and are presented in Figs. 4.10a, 4.10b, and $4.10 \mathrm{c}$, respectively. The waveguides had grating duty ratios of $61 \%$ and $82.5 \%$ for $\mathrm{TE}$ and TM polarized light respectively. They were set to these conditions as these waveguides show nearly athermal behavior. 
(a)

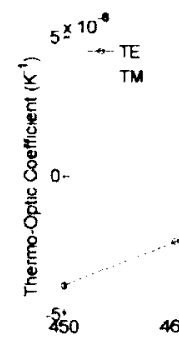

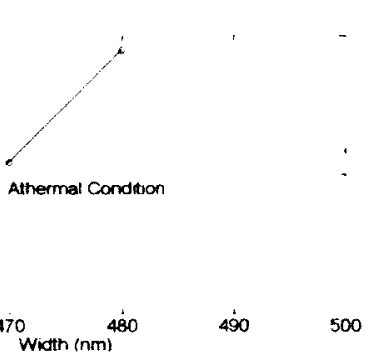

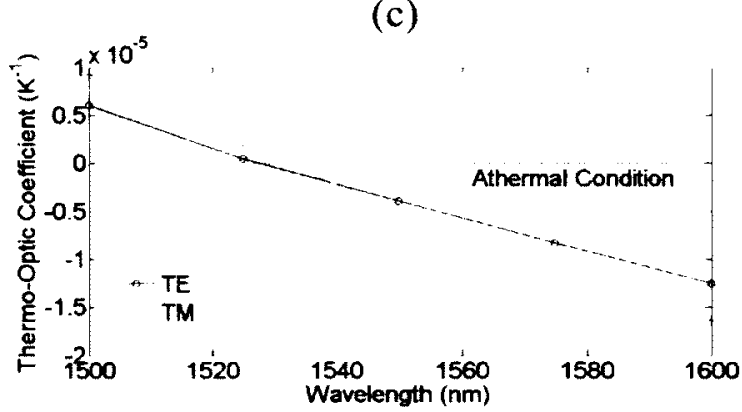

(b)

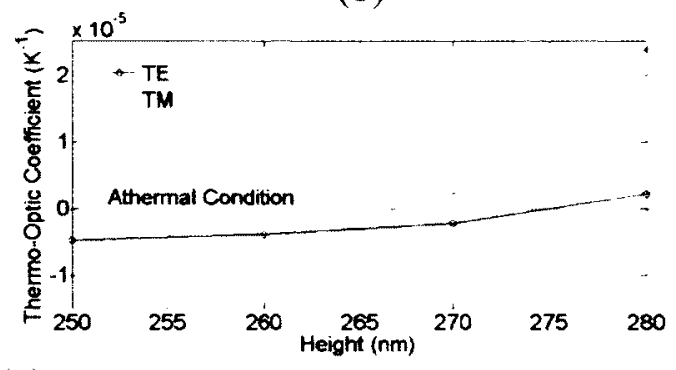

(c)

.


Figure 4.10 The TO coefficient dependence on the SWG waveguide parameters

The TO coefficient dependence on the waveguide (a) width, (b) height, and (c) operating wavelength near athermal behaviour (SWG)

From the results shown in Fig. 4.10, we have determined the athermal performance of the reference waveguide through the influence of variations in the waveguide height, width, and operating wavelength. Changes in the TO coefficient per nanometer change in the waveguide width near athermal condition are approximately $2.8 \times 10^{-7} \mathrm{~K}^{-1} / \mathrm{nm}$, and $1.2 \mathrm{x}$ $10^{-7} \mathrm{~K}^{-1} / \mathrm{nm}$ for TE and TM polarizations, respectively. This means that if we assume a fabrication tolerance of $10 \mathrm{~nm}$ for the waveguide width, the TO coefficient of the SWG waveguide would be in the low $10^{-6} \mathrm{~K}^{-1}$-range, two orders of magnitude smaller than the TO coefficient of a conventional photonic wire waveguide. Changes in the TO coefficient per $1 \mathrm{~nm}$ change in the waveguide height are approximately $2.3 \times 10^{-7} \mathrm{~K}^{-1} / \mathrm{nm}$ (TE), and $6.2 \times 10^{-7} \mathrm{~K}^{-1} / \mathrm{nm}$ (TM polarization). Finally, changes in the TO coefficient per $1 \mathrm{~nm}$ 
change in the operating wavelength close to the athermal point are approximately $-1.8 \mathrm{x}$ $10^{-7} \mathrm{~K}^{-1} / \mathrm{nm}$ for TE polarized light, and $-2.5 \times 10^{-7} \mathrm{~K}^{-1} / \mathrm{nm}$ for TM polarized light, giving us insight on the SWG waveguide "athermal" bandwidth.

\subsection{Design of Athermal BSWG Waveguides}

Due to the required small dimensions, SWG waveguides with high grating duty ratios are difficult to fabricate since they require patterning techniques with high resolution (less than $100 \mathrm{~nm}$ gaps). If the lithographic resolution is insufficient, fabricated SWG waveguides with small gaps will often resemble conventional photonic wire waveguides with duty ratios of $100 \%$. In addition, Figs. 4.6 and 4.8 show that both height and width would have to increase in order to reduce the athermal duty cycle. We must remember that in practice, it is not ideal to do so as larger waveguides may no longer meet the single-mode condition. Waveguide dimensions are also often restricted due to manufacturing processes and finally, smaller, cheaper and faster chips are preferable. Consequently, an alternative configuration was proposed for guiding TE, but especially TM polarized light where duty ratios can be kept close to $50 \%$ even for wider waveguides (see Fig. 4.6). The bridged subwavelength grating waveguide (BSWG) now replaces the small gaps by narrower core segments of width $\mathrm{W}_{2}$. A two-dimensional schematic top view of the new proposed structure is depicted in Fig. 4.11 below. The bridged subwavelength grating waveguide is comprised of the same two materials: silicon and SU-8 polymer. The substrate is silicon dioxide ( $\mathrm{SiO} 2)$. 

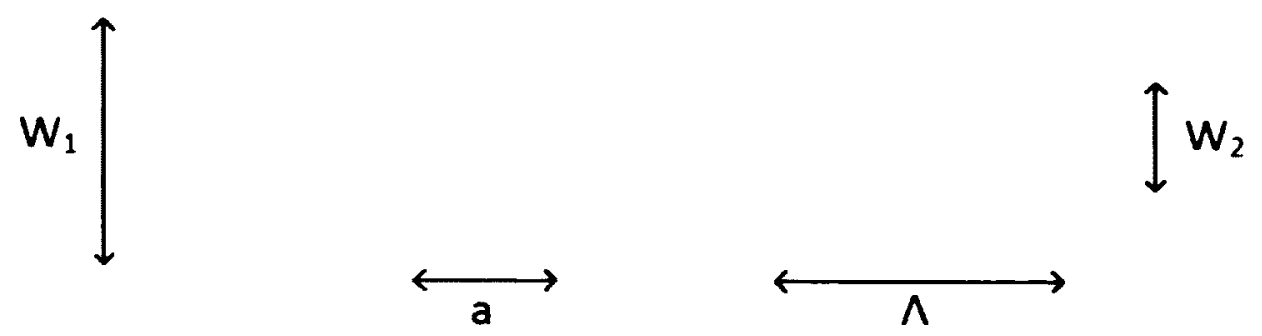

Figure 4.11 Two-dimensional Schematic top view of the BSWG waveguide

The BSWG waveguide parameters are illustrated here. $W_{l}$ is the waveguide width, i.e. the width of the wider silicon core segments, $W_{2}$ is the width of the narrower silicon segments replacing the polymer-filled gaps of the SWG waveguides, $a$ is the length of the wider segments, and $A$ is the grating pitch. The grating's duty cycle is defined as $a / A$, the length of one of the wider segments over the pitch.

\subsubsection{MPB Code}

The MPB script shown in section A.3 describes the structure of a BSWG waveguide. We notice that there is a new defined parameter there, namely the bridge width $\mathrm{W}_{2}$. The bridge width is defined as the width of the smaller segment in that script. The narrower segments of width $W_{2}$ have been added to the unit cell to fill out the former gaps. The bridge width of the BSWG structure defined in the script is $40 \mathrm{~nm}(0.04 \mu \mathrm{m})$. The height, width, and grating pitch of the structure are $490 \mathrm{~nm}, 260 \mathrm{~nm}$, and $250 \mathrm{~nm}$, respectively. The length of the bigger and smaller segments that are in the left and right of the unit cell are $145 \mathrm{~nm}$, and $52.5 \mathrm{~nm}$, respectively. The grating duty cycle $(\mathrm{a} / \Lambda)$ of this $\mathrm{BSWG}$ structure is therefore $58 \%$. The length of the three slabs (left, middle, and right) should add up to the grating pitch as shown in Fig. 4.12. 


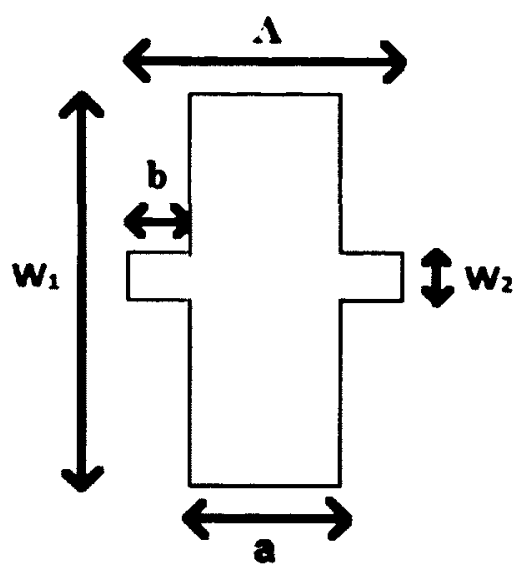

Figure 4.12 Two-dimensional schematic top view of the BSWG (unit cell)

The waveguide width $\left(W_{1}\right)$ and grating pitch $(\Lambda)$ are $490 \mathrm{~nm}$ and $250 \mathrm{~nm}$, respectively. The length of the wide (a) and small (b) segments is $145 \mathrm{~nm}$ and $52.5 \mathrm{~nm}$, respectively (DC $=58 \%$ ). The bridge width $\left(\mathrm{W}_{2}\right)$ in this example is $40 \mathrm{~nm}$.

The dielectric constants that are defined here are the ones at room temperature $(\mathrm{T}=$ 293K). To simulate a temperature change, we can again refer to equation (4.1) to find the temperature-dependent refractive indices and dielectric constants of the corresponding materials.

As opposed to the ordinary SWG structure, additional blocks have also been added since we now have narrower segments to fill out the former gaps. The additional code is in bold in the script in order to make the commands more prominent to the reader.

Moreover, explanations on how to use the MPB file that is generated from the simulation are explained in Chapter 3 and Appendix A. An example file containing the telecommunications frequencies (around $\mathrm{f}=193.55 \mathrm{THz}$ ) of the TM fundamental mode 
that is propagating inside the BSWG structure with $W=450 \mathrm{~nm}, \mathrm{H}=260 \mathrm{~nm}, \Lambda=250$ $\mathrm{nm}$, and $\mathrm{DC}=62 \%$ is shown in section A.4.

\subsubsection{Analysis and Procedure}

The procedure for finding the waveguide's effective thermo-optic coefficient remains essentially the same as described in the previous section. A simple plot illustrating the dependence of the effective index on the temperature is sufficient. The TO coefficient is obtained from the slope of the plot's regression (best fit) line. An example plot is shown below (Fig. 4.13).

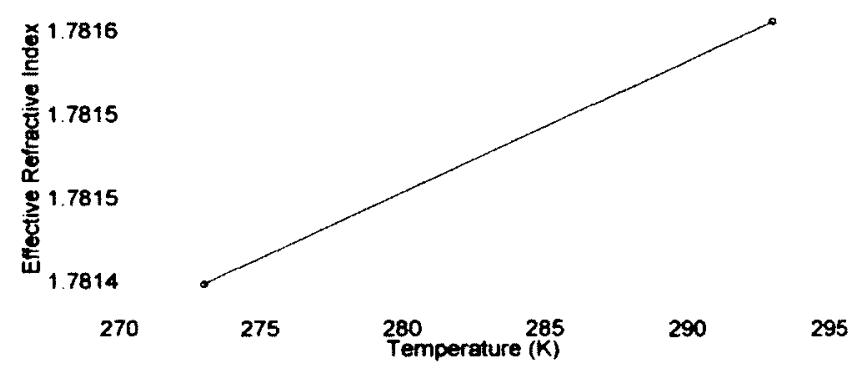

Figure 4.13 The effective refractive index dependence on the temperature

The waveguide width, height, grating pitch, and grating duty cycle here are $450 \mathrm{~nm}, 260 \mathrm{~nm}, 300 \mathrm{~nm}$, and $50 \%$ respectively. The bridge width is $150 \mathrm{~nm}$. Light is TM polarized with a wavelength of $1550 \mathrm{~nm}$

The slope $(\mathrm{m})$ of this particular regression line is $\mathrm{m}=7.873 \times 10^{-6} \mathrm{~K}^{-1}$. The TO coefficient was found for varying bridge widths. 


\subsubsection{Simulation Results}

The TO coefficient dependence on the bridge width (sidewall segment width) for a waveguide with a width of $450 \mathrm{~nm}$, a height of $260 \mathrm{~nm}$, a grating pitch of $300 \mathrm{~nm}$ and a duty cycle of $50 \%$ is shown in Fig. 4.14 . During the analysis, the grating pitch was first set to $300 \mathrm{~nm}$, and then set to $250 \mathrm{~nm}$ since our fabricated BSWG structures had a grating pitch of $250 \mathrm{~nm}$. A grating pitch of $250 \mathrm{~nm}$ was chosen for all experimental SWG and BSWG structures to avoid the possibility of Bragg reflections in the coupler regions (see Chapter 5). The grating pitch and duty cycle of the waveguide in Fig. 4.14 is $300 \mathrm{~nm}$ and $50 \%$ respectively. Investigations on the tolerance of the temperature-independent BSWG waveguides to variations in the other parameters were then carried out using that waveguide as the reference waveguide. Investigations on the effect of changing the bridge width for other various duty cycles to the waveguide's TO coefficient is shown in Fig. 4.15. The grating pitch of the waveguides shown in Fig. 4.15 was set to $250 \mathrm{~nm}$, the actual grating pitch of the fabricated BSWG structures.

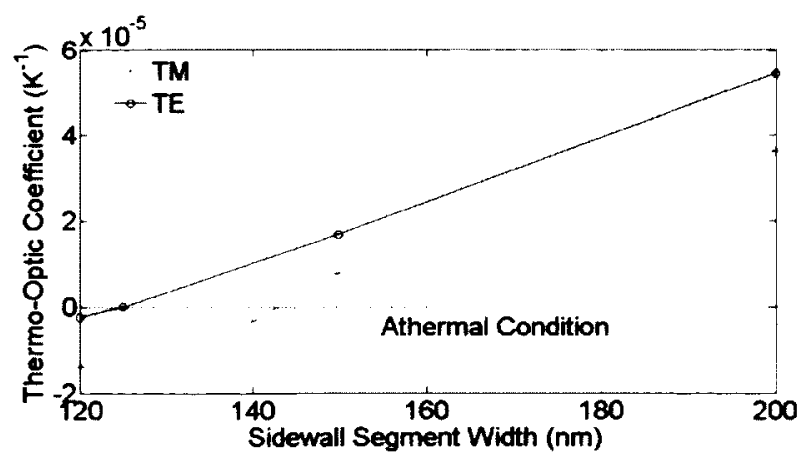

Figure 4.14 The BSWG waveguide TO coefficient dependence on the bridge width

The waveguide width, height, grating pitch, and grating duty cycle are $450 \mathrm{~nm}, 260 \mathrm{~nm}, 300 \mathrm{~nm}$, and $50 \%$ respectively. The operating wavelength is $1550 \mathrm{~nm}$. 



Figure 4.15 The TO coefficient dependence on the bridge width

The thermo-optic coefficient dependence on the bridge width for BSWG waveguides with duty cycles of (a) $35 \%$ (b) $42.5 \%$ (c) $50 \%$ (d) $57.5 \%$ and (e) $65 \%$ near athermal behavior. The waveguide width, height, and grating pitch are $450 \mathrm{~nm}, 260 \mathrm{~nm}$, and $250 \mathrm{~nm}$ respectively. The operating wavelength is $1550 \mathrm{~nm}$.

Interesting conclusions can be drawn from the plots of Fig. 4.15. First of all, several temperature independent BSWG waveguide designs exist. For a fixed duty cycle, there is a required specific bridge width that depends on the polarization of light. For example, a $35 \%$ duty cycle temperature independent BSWG waveguide with a grating pitch of 250 
$\mathrm{nm}$ requires a bridge width of $200 \mathrm{~nm}$ for TE, and $190 \mathrm{~nm}$ for TM polarized light. A bridge width of $135 \mathrm{~nm}$ for TE and $160 \mathrm{~nm}$ for TM is needed if the duty cycle of the BSWG waveguide is $50 \%$. If the grating pitch is $300 \mathrm{~nm}$, then a new thermal design is necessary. The bridge width required in that case is $125 \mathrm{~nm}$ for TE and $143 \mathrm{~nm}$ for TM polarized light. The bridge width dependence on the waveguide's duty cycle for temperature-independent BSWG waveguides with a grating pitch of $250 \mathrm{~nm}$ is summarized in Fig. 4.16.

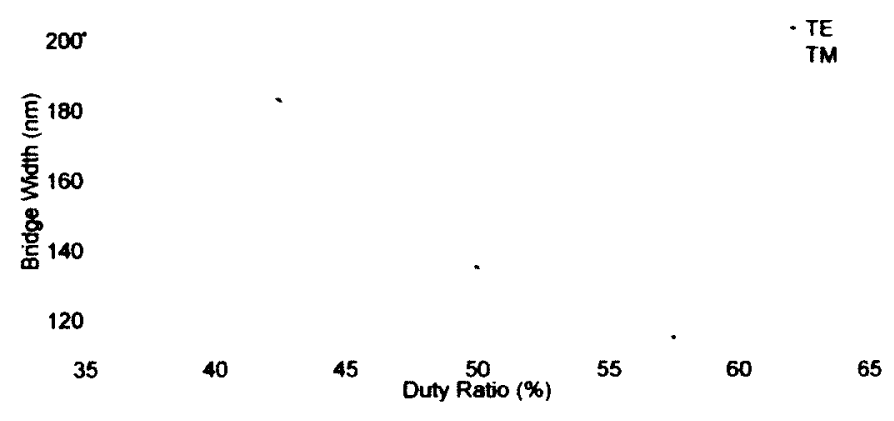

Figure 4.16 The bridge width dependence on the duty ratio

The bridge width dependence on the duty ratio for athermal BSWG waveguides. The waveguide width, height, and grating pitch are $450 \mathrm{~nm}, 260 \mathrm{~nm}$, and $250 \mathrm{~nm}$ respectively. The operating wavelength is 1550

$\mathrm{nm}$.

Interestingly, Fig. 4.16 shows that the realization of waveguides which are temperature independent for both polarizations is also possible with the new periodic bridged structure. For waveguides operating at $\lambda=1550 \mathrm{~nm}$ with a width, height and grating pitch of $450 \mathrm{~nm}, 260 \mathrm{~nm}$, and $250 \mathrm{~nm}$, the BSWG waveguide requires a duty cycle of $44 \%$ and a bridge width of about $175 \mathrm{~nm}$. Their effective refractive indices are however not the same for both polarizations $\left(\mathrm{n}_{\mathrm{TE}}=1.87, \mathrm{n}_{\mathrm{TM}}=1.77\right)$. The implementation of an athermal 
waveguide that works for both polarizations may represent a big advantage in the world of telecommunications.

\subsubsection{BSWG Waveguide Tolerance and Thermal Bandwidth}

Variations of the thermo-optic effect near athermal operation due to small changes in width, height, and operating wavelength are illustrated in Fig. 4.17. The waveguide width, height, and grating pitch were at first set to $450 \mathrm{~nm}, 260 \mathrm{~nm}$, and $300 \mathrm{~nm}$. The sidewall segment widths were set to $125 \mathrm{~nm}$ for TE polarized light, and $143 \mathrm{~nm}$ for TM polarized light such that athermal conditions are met.

(a)
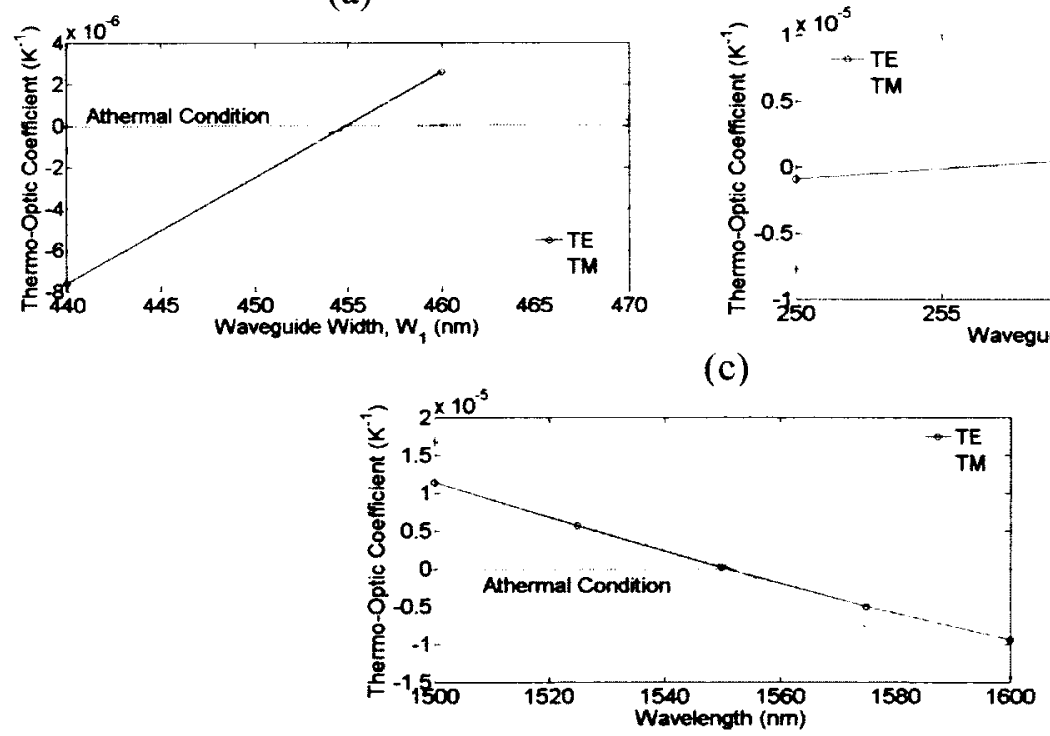

Figure 4.17 The TO coefficient dependence on the BSWG waveguide parameters

The TO coefficient dependence on (a) the BSWG waveguide width, (b) height, and (c) operating wavelength near athermal behavior

For this structure, changes in the TO coefficient per nanometer change in the waveguide width close to athermal conditions are approximately $5.1 \times 10^{-7} \mathrm{~K}^{-1} / \mathrm{nm}$, and $5.0 \times 10^{-8} \mathrm{~K}^{-}$ 
1/nm for TE and TM polarizations, respectively. Changes in the TO coefficient per $1 \mathrm{~nm}$ change to the waveguide height are approximately $1.5 \times 10^{-7} \mathrm{~K}^{-1} / \mathrm{nm}$, and $8.4 \times 10^{-7} \mathrm{~K}^{-}$ $1 / \mathrm{nm}$ for TE and TM polarizations, respectively. Changes in the TO coefficient per $1 \mathrm{~nm}$ change in the operating wavelength are approximately $-2.1 \times 10^{-7} \mathrm{~K}^{-1} / \mathrm{nm}$ for $\mathrm{TE}$ polarized light, and $-3.1 \times 10^{-7} \mathrm{~K}^{-1} / \mathrm{nm}$ for TM polarized light. Note that the latter changes are slightly greater than, but of the same order of magnitude as the changes in the TO coefficient of the SWG waveguides considered in the previous section. In other words, the athermal performance of the BSWG waveguide is slightly more sensitive to variations in the waveguide height, width, and operating wavelength than the SWG waveguides. Yet, the fabrication requirements are more relaxed as the critical dimensions are significantly larger. TO effect variations due to small changes in the sidewall segment width were also calculated numerically. Changes in the TO coefficient per $1 \mathrm{~nm}$ change in the narrow sidewall segment width are approximately $2.6 \times 10^{-7} \mathrm{~K}^{-1} / \mathrm{nm}$, and $7.3 \times 10^{-7}$ $\mathrm{K}^{-1} / \mathrm{nm}$ for TE and TM polarizations respectively as shown in Fig.4.18.

(a) TE Polarization

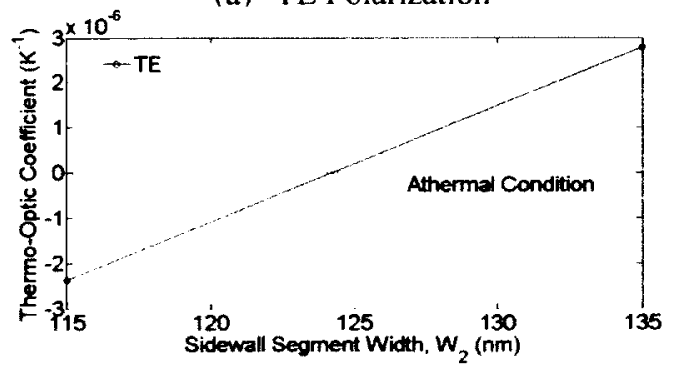

(b) TM Polarization

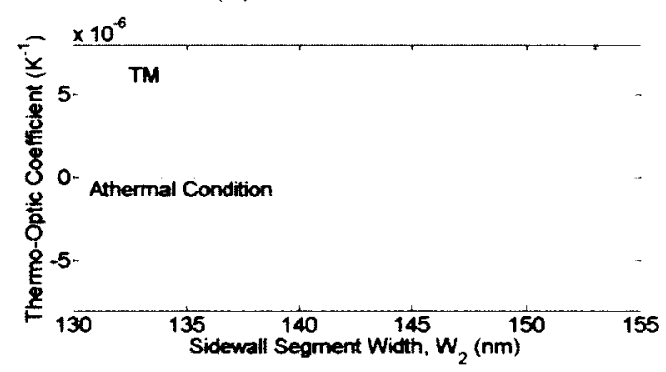

Figure 4.18 The TO coefficient dependence on the bridge width (TE and TM)

The TO coefficient dependence on the bridge width (sidewall segment width), $W_{2}$ for (a) TE, and (b) TM polarizations 
We have presented several designs of athermal waveguides with various waveguide widths, heights, and periodicities. The designs also depend on the polarization of light. In addition, the TO coefficient of both temperature-independent SWG and BSWG structures is quite sensitive to variations in the waveguide parameters. However, even if the actual dimensions of the sample waveguides are slightly different than the nominal ones, their thermo-optic coefficient remains much smaller than silicon's TO coefficient value. The waveguides are hence 10 to 20 times less temperature-dependent than silicon photonic wire waveguides (see Chapter 5). In many applications, such waveguides are still considered to be sufficiently athermal. 


\section{Chapter: Demonstration of Athermal SWG/BSWG Waveguides}

This chapter covers the mask layout of our sample, the experimental setup, as well as the experimental results for the SWG and BSWG waveguide's effective thermo-optic (TO) coefficient. The experimental results are then compared to the theoretical calculations predicted by the MPB software as discussed in Chapter 4 .

\subsection{The Fabrication and Realization of Athermal SWG Waveguides}

\subsubsection{SWG Waveguide Mask Layout}

Before fabricating the device and recording the data, the first step consists of designing the waveguide test structures and the sample layout. The layout for measuring the TO coefficient of diverse SWG structures was designed by Dr. Jens Schmid. The mask layout was drawn using AutoCAD. A screenshot of the bottom, middle, and top part of the mask layout is shown below. 
(a)

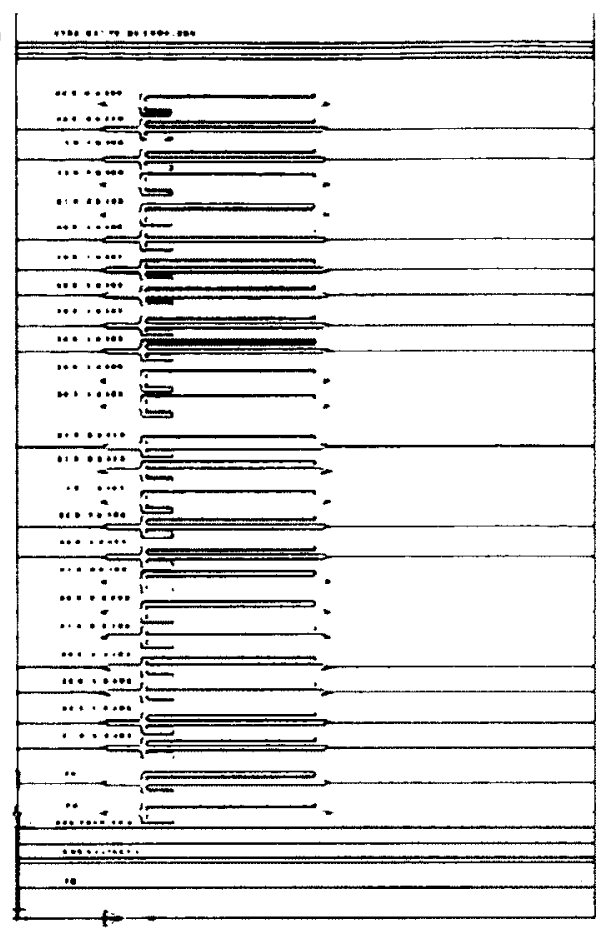

(b)

(c)

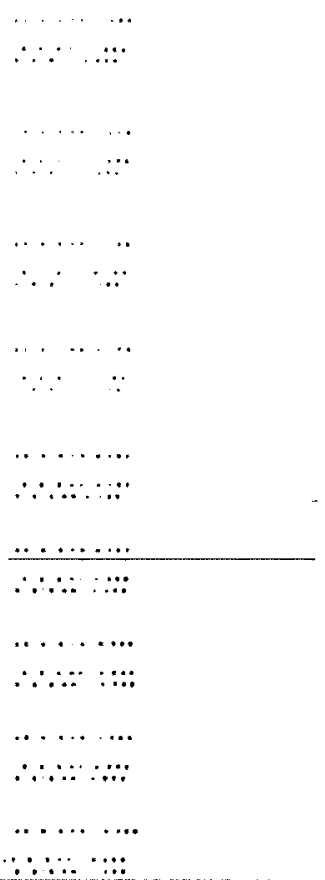

Figure 5.1 Screenshot of the SWG mask layout

A screenshot of the (a) bottom, (b) center, and (c) top part of the SWG mask layout. Various unbalanced Mach-Zehnder interferometers with varying grating duty cycles are shown in (a). SWG waveguides of different lengths and widths used for loss measurements are shown in (b) and (c).

To measure the effective TO coefficient of the SWG waveguides, the SWG structures are incorporated into Mach-Zehnder interferometer (MZI) devices as shown in Fig. 5.1a and 5.2. The $\mathrm{Y}$ splitters and waveguide bends comprise photonic wire (PW) waveguides while each arm of the MZIs comprises straight SWG structures. One arm of the MZI is longer than the other by $3 \mathrm{~mm}(\Delta \mathrm{L}=3 \mathrm{~mm})$ creating an interference pattern. An optical transmission spectrum will be collected at the output. Coupling structures such as the one shown in Fig. 5.3 [50] are used to adiabatically transform the PWs into SWGs demonstrating the compatibility of the two types of waveguides. The location of these 
coupling structures is indicated by the arrows in Fig. 5.2. The sample layout also includes various microphotonic SWG waveguides of different lengths in order to measure the propagation loss of the SWG structures (loss structures). The waveguide bends are comprised of photonic wire waveguides since the bending loss of the SWG waveguides is currently not well understood, which would complicate the analysis of loss data. A schematic of these added structures is shown in Fig. 5.1b-c and 5.4. For a given waveguide width, they were $5.875 \mathrm{~mm}, 15.875 \mathrm{~mm}$, or $25.875 \mathrm{~mm}$ long. The loss structures have been included in the layout in order to determine whether the propagation loss is dependent on the waveguide width.

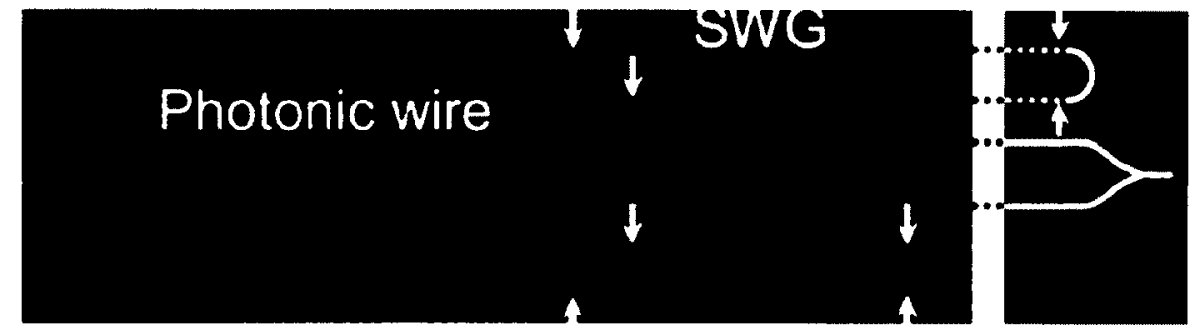

Figure 5.2 Schematic of the unbalanced MZI device with SWG sections

A schematic of the unbalanced Mach-Zehnder device with SWG sections [2]. The arrows indicate the positions of the PW-SWG waveguide couplers.

(a)

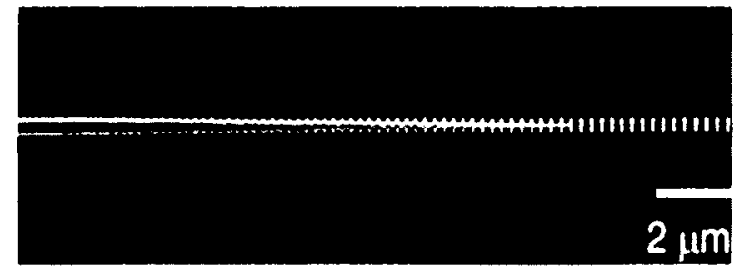

(b)

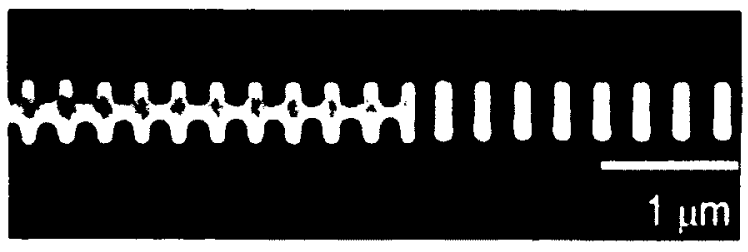

Figure 5.3 SEM images of PW-SWG waveguide couplers

The PW waveguides adiabatically transform the PWs into SWGs. 
(a)

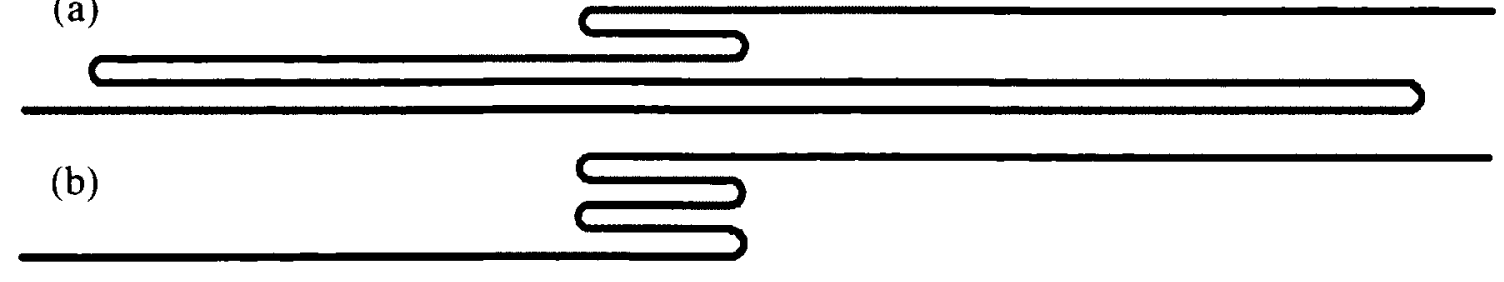

Figure 5.4 Schematic of two microphotonic waveguides

The straight sections are comprised of SWGs. Various loss structures are included in the sample in order to measure the propagation loss of the SWGs. The waveguide in (a) is $15.875 \mathrm{~mm}$ long and the waveguide in

(b) is $5.875 \mathrm{~mm}$ long.

\subsubsection{Fabrication}

The SWG waveguides are silicon-on-insulator (SOI) waveguides [60]. The buried oxide layer is $2 \mu \mathrm{m}$ thick and the silicon waveguide layer in which the SWG core segments are made, is $0.26 \mu \mathrm{m}$ thick. The height of the waveguides is consequently fixed to $260 \mathrm{~nm}$, a typical value for silicon photonic wire waveguides. This is the reason why we have designed our waveguides to have a height of $260 \mathrm{~nm}$ in our simulations (see Chapter 4).

The MZIs, loss structures, and SWG structures are fabricated by electron beam lithography and plasma reactive ion etching. They are coated with a $2 \mu \mathrm{m}$-thick layer of SU-8 polymer. The SU-8 polymer layer was placed by the standard spin and bake procedure. The sample was fabricated in the NRC nanofabrication facility by Dr. Jean Lapointe and Dr. Jens H. Schmid. Dr. Jean Lapointe was responsible for electron beam lithography and Dr. Jens Schmid for the other fabrication steps of the sample. 


\subsubsection{Experimental Setup}

To measure the thermo-optic coefficient of the SWG structures, the optical transmission spectrum is collected at the output at several temperatures. The samples are mounted on an adjusting optical bench with a temperature-controlled copper heat sink. The TE or TM polarized light is coupled into the MZIs and loss structures via optical fiber. A microscope objective is used to collect the transmitted light at the waveguide output. A picture of the experimental setup is shown in Fig. 5.5 .



Figure 5.5 Picture of the experimental setup

A picture of the experimental setup. The chip is mounted on a copper heat sink. Light is coupled into the chip via optical fiber.

\subsubsection{Analysis and Procedure}


Figure 5.6 shows several optical transmission MZI curves at different temperatures of two devices with different SWG duty ratios. The first MZI is comprised of photonic wire (PW) waveguides and the other, with SWG structures with a grating duty cycle of $66 \%$. Photonic wire waveguides can be considered as SWG structures with a grating duty ratio of $100 \%$. The effective thermo-optic (TO) coefficient of the SWG structure can be measured from the wavelength shift of the MZI spectrum due to the change in temperature [61]:

$$
\frac{\delta \lambda}{\delta T}=\frac{\lambda_{Q}}{n_{g}} \frac{g n_{o f f}}{\delta T}
$$

Where $n_{\text {eff }}$ is the effective refractive index of the waveguide, $n_{g}$ is its group index and $\lambda_{o}$ is the operating wavelength in vacuum. The group index, defined as the speed through which the embedded information travels within the medium (see Chapter 3), can be determined from the MZI spectrum. If the difference in length $(\Delta \mathrm{L})$ between the two MZI arms and the distance between two successive minima closest to the operating wavelength $(\Delta \lambda)$ are known, the group index can be calculated using the following relationship:

$$
n_{q}=\frac{\lambda_{q}^{2}}{\Delta \lambda \Delta L}
$$


Finally, by combining equations 5.1 and 5.2 , we get an interesting equation that relates the TO coefficient to the temperature-induced shift, the difference in length between the two MZI arms, the operating wavelength, and the distance between two successive MZI minima at the operating wavelength.

\section{$\frac{\delta \lambda}{\delta T}-\frac{\Delta \lambda \Delta L}{\lambda_{0}} \frac{\delta n_{0 \ell}}{\delta T}$}

The TO coefficient at each minimum of the MZI optical spectrum was calculated using equation (5.3). We were able to measure the TO coefficient of all the waveguides at many wavelengths over a $50 \mathrm{~nm}$ spectral range $(1530-1580 \mathrm{~nm})$ giving us insight on the variations of the TO coefficient as a function of the operating wavelength, i.e. the measured SWG waveguide "thermal" bandwidth (see section 4.1.4). The calculations for measuring the TO coefficient from the wavelength shift at each minimum for all waveguides were carried out in MATLAB (by Dr. J.H. Schmid).

Figure 5.6 shows the spectral transmittance for TE polarization at $\lambda \sim 1550 \mathrm{~nm}$ of the MZI devices with SWG waveguides of varying duty cycles at different temperatures. The fabricated SWG waveguides have a width, height, and grating pitch of $470 \mathrm{~nm}, 260 \mathrm{~nm}$, and $250 \mathrm{~nm}$ respectively. Different temperature-induced wavelength shifts corresponding to waveguides with different grating duty cycles are observed. For example, we notice, from Fig. 5.6c, that the temperature-induced wavelength shift is substantially smaller for the SWG with a duty cycle of $66 \%$. A sign reversal in the wavelength shift is also 
observed in (a) and (b) of Fig. 5.6. The TO coefficient of the SWG waveguide with DC = $46 \%$ is thus negative since the temperature-induced spectral shift is to the left with respect to the spectrum of the reference waveguide measured at a temperature of $20^{\circ} \mathrm{C}$. A wavelength shift of approximately $70 \mathrm{pm} /{ }^{\circ} \mathrm{C}$ is observed when the grating duty cycle is $100 \%$ (PW waveguide).
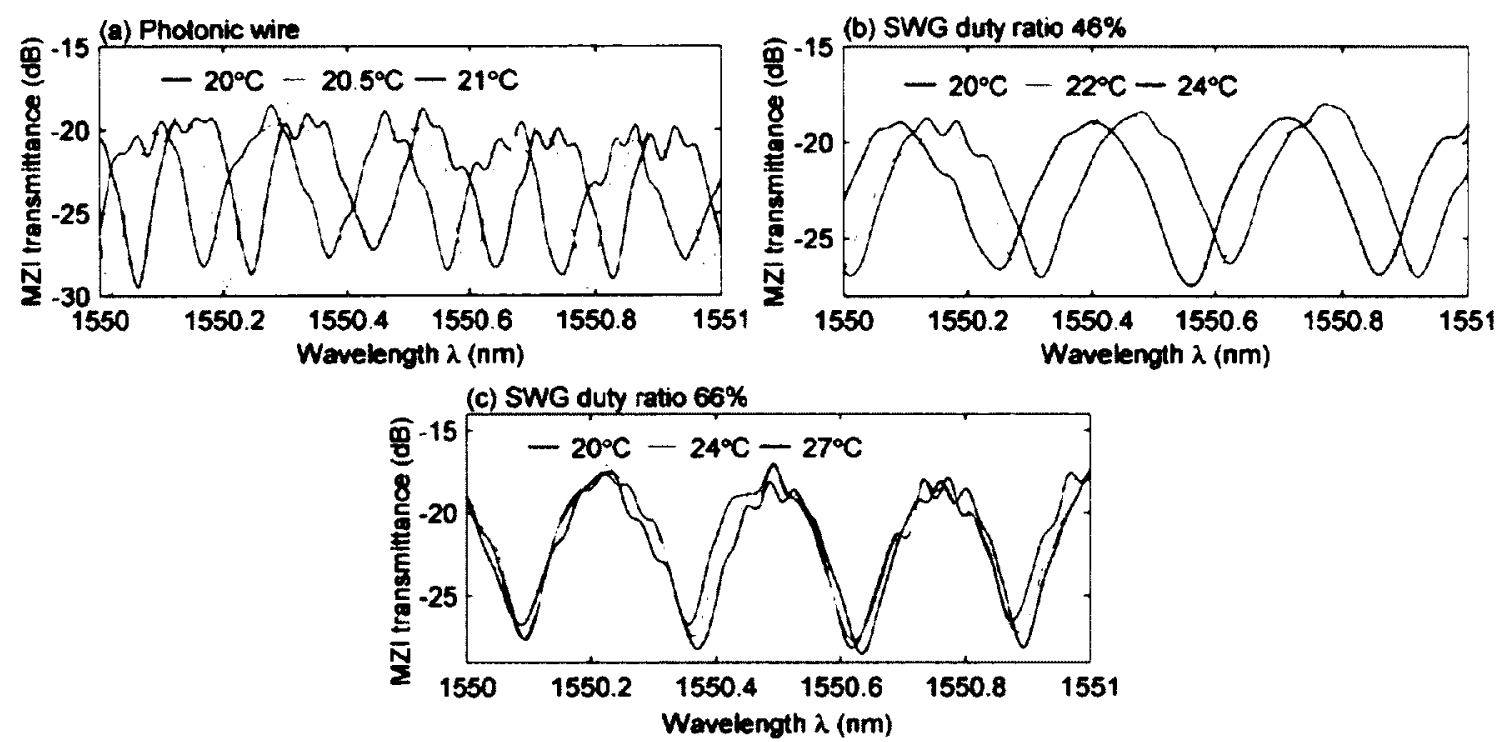

Figure 5.6 The dependence of the MZI transmittance on the wavelength

The dependence of the MZI transmittance on the wavelength at several temperatures for different waveguide configurations. The arms of the MZIs here comprise (a) photonic wires, (b) SWG waveguides with $\mathrm{DC}=46 \%$, and (c) SWG waveguides with $\mathrm{DC}=66 \%$. The wavelength shift is substantially smaller for the SWG waveguide with a duty cycle of $66 \%$. The guided light is TE polarized

As mentioned before, the TO coefficient of all the waveguides in the sample was calculated from the observed temperature-induced spectral shifts of the transmission minima. The calculations for the TO coefficient of the SWG waveguides for both TE and TM polarizations were carried out by Dr. J. H. Schmid. The results are plotted in Fig. 5.7 
below. The data points shown in that figure correspond to the measured spectral shift of each minima. The solid lines are linear fits to the data.

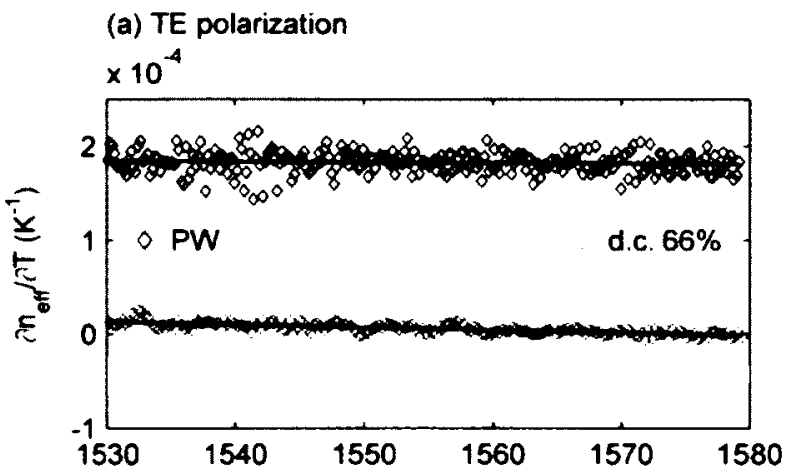

(b) TM polarization

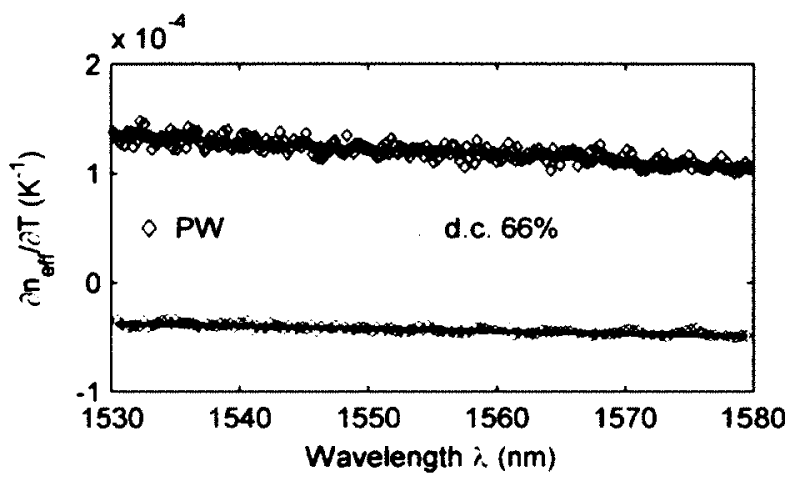

Figure 5.7 The TO coefficient dependence on the wavelength (PW and SWG)

The TO coefficient dependence of a photonic wire (PW) waveguide and a $66 \%$ DC SWG waveguide on the wavelength. Black solid lines are linear fits. (a) TE polarization, (b) TM polarization

According to Fig. 5.7, the SWG waveguide with a $66 \%$ grating duty cycle is nearly athermal for TE polarization. The results shown above are thus consistent with the very small spectral shifts observed in Fig. 5.6c. The TO coefficient of the photonic wire waveguide is great and positive in concordance to what is observed in Fig. 5.6a. For TM polarization however, the TO coefficient of the $\mathrm{PW}$ waveguide is again positive, but is negative for the $66 \%$ DC SWG waveguide. 
In reality, none of our sample SWG waveguides exhibited athermal behavior for TM polarization, but from the sign reversal of Figs. 5.6 and 5.7, it is obvious that athermal behavior for the SWG waveguides will exist if the grating duty cycle is between $66 \%$ and $100 \%$ as predicted by the theory (see section 4.1 .3 , and section 5.1 .5 ). Yet, as mentioned before, waveguides with such high grating duty cycles are difficult to fabricate and the BSWG structure is therefore introduced.

\subsubsection{Comparison with MPB Simulations}

The TO coefficients of the linear fits shown in Fig. 5.7 at $\lambda=1550 \mathrm{~nm}$ are plotted as functions of the waveguide grating duty cycle for TE and TM polarizations in Fig. 5.8 below. These are compared with the MPB simulation results shown below. Additional simulations were carried out since our fabricated waveguides were $470 \mathrm{~nm}$ wide, as measured by scanning electron microscopy on the fabricated samples, i.e. $20 \mathrm{~nm}$ wider than the design.

Fig. 5.8 includes the measured TO coefficient values of SWG waveguides with duty cycles of $46 \%, 56 \%$, and $64 \%$. The former two have smaller TO coefficients than the PW waveguides, but are not as athermal as the SWG waveguides with DCs of $64 \%$ or $66 \%$.

Our lowest measured TO coefficient value, as shown below, is $1.8 \times 10^{-6} \mathrm{~K}^{-1}$ which corresponds to an SWG waveguide with a duty cycle of $64 \%$. 
The experiment confirms the expected increase of the TO coefficient with increasing grating duty cycle as mentioned in Chapter 4 . Near athermal behavior is observed around $64 \%$ and $84 \%$ for TE and TM polarizations, respectively. Overall, the experimental data is in good agreement with the numerical simulations. The results hence validate our strategy of mitigating the TO coefficient with polymer cladding and subwavelength patterning.

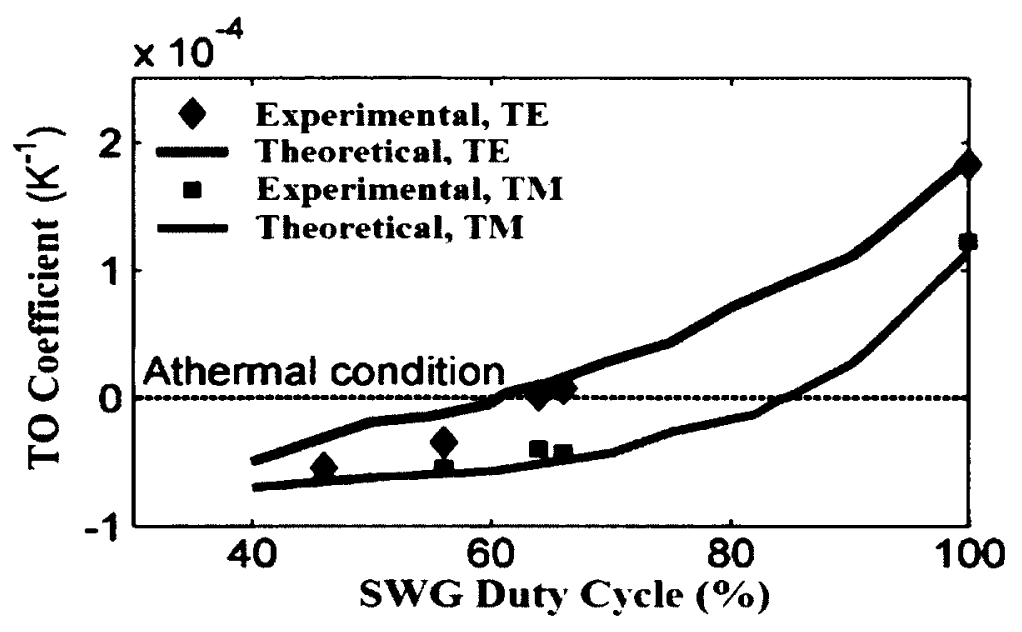

Figure 5.8 The effective TO coefficient dependence on the SWG duty cycle

The effective TO coefficient dependence on the SWG grating duty cycle. The experimental results are compared with the MPB theoretical simulations.

\subsubsection{SWG Loss Measurements}

Investigations on the propagation loss of the SWG structures were carried out. We have calculated the loss by measuring the power at the output of the loss structures (see Fig. 5.4). The loss structures had varying lengths and widths in order to see whether the loss is dependent on such parameters. The propagation loss for a given SWG waveguide width was obtained from a linear fit to the data of the power dependence on the structure's 
length. An example plot is shown in Fig. 5.9 below. The structures had a nominal height, grating pitch, and grating duty cycle of $260 \mathrm{~nm}, 250 \mathrm{~nm}$ and $50 \%$ respectively. The results describing the propagation loss of the SWG waveguides for all waveguide widths for both TE and TM polarizations are tabulated below (table 5.1). These data fits have been carried out for three different samples in order to provide more accuracy and stability in the measurements.

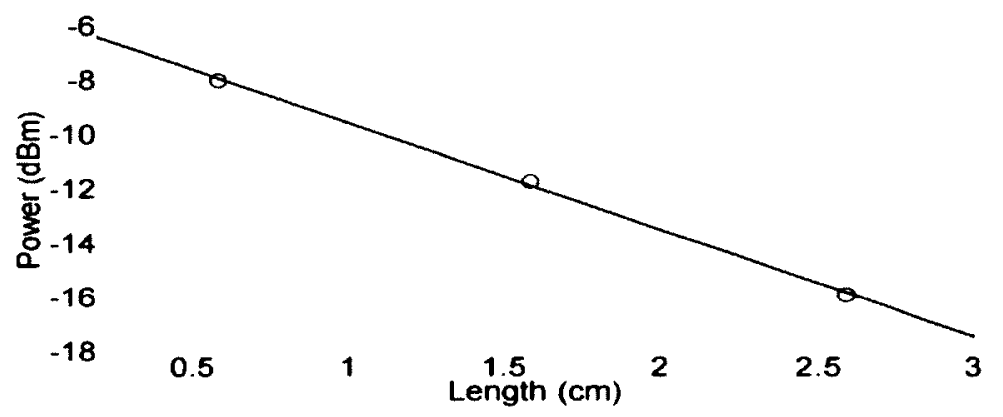

Figure 5.9 The output power dependence on the length of an SWG structure

The SWG structures had a nominal height, grating pitch, and grating duty cycle of $260 \mathrm{~nm}, 250 \mathrm{~nm}$, and $50 \%$. This particular structure was nominally $450 \mathrm{~nm}$ wide $\left(1^{\text {st }}\right.$ sample). The propagation loss is found by calculating the slope of the linear fit (black solid line). The slope of this graph is $-3.9 \mathrm{~dB} / \mathrm{cm}$. 


\begin{tabular}{|c|c|c|c|c|c|c|}
\hline \multirow{2}{*}{$\begin{array}{c}\text { SWG Waveguide Width } \\
\text { (nm) }\end{array}$} & \multicolumn{3}{|c|}{ Propagation Loss (TE) $(\mathrm{dB} / \mathrm{cm})$} & \multicolumn{3}{|c|}{ Propagation Loss (TM) (dB/cm) } \\
\hline & Sample 1 & Sample 2 & Sample 3 & Sample 1 & Sample 2 & Sample 3 \\
\hline 200 & - & & & & 4.3 & 0.2 \\
\hline 250 & 3.8 & 7.6 & 1.7 & 3.5 & & 0.9 \\
\hline 300 & 5.6 & 5.0 & 4.8 & 4.0 & 4.5 & 2.1 \\
\hline 350 & & 2.6 & & & 4.7 & 1.6 \\
\hline 400 & 6.3 & $=$ & 6.3 & 5.3 & 7.0 & 2.6 \\
\hline 450 & 10.6 & $\bar{E}$ & 3.9 & 3.9 & & 3.2 \\
\hline 500 & $\overline{-}$ & $=$ & ב- & 6.2 & 8.2 & 3.9 \\
\hline 550 & $=$ & $\overline{-}$ & - & 5.7 & 6.2 & 4.0 \\
\hline 600 & - & $=$ & - & 4.9 & 3.6 & 2.5 \\
\hline
\end{tabular}

Table 5.1 The propagation loss as a function of the SWG waveguide width (TE, TM)

The measurements presented here are extracted from the first, second, and third samples being tested. In some cases, the measurements were inconsistent and have been disregarded (see black solid lines above).

The data of the three samples is not consistent, and we therefore have to conclude that there is a significant error in the measurements. Through the usage of an IR camera, looking at the sample surface from above while light was propagating in the waveguide, we were able to identify one of the reasons for such discrepancies. It was apparent that there was a lot of light scattering mainly in the areas of the couplers and PW-SWG mode transformers, especially for TE polarization. The couplers seemed to scatter less light and generally work better for TM polarization, not only for measuring the loss, but also for measuring the TO coefficients of the SWG structures. For example, we have consistently observed less noise in the MZI transmission spectra for TM polarization throughout the experiment (see Figs. 5.6 and 5.15); however, it is not clear whether this fact is related to the observed larger scattering from the coupler structures. The PW-SWG mode transformers generated additional scattering. Nonetheless, we can see that our waveguides of interest $(W=450 \mathrm{~nm})$, on average, have a propagation loss of about 3.5 
$\mathrm{dB} / \mathrm{cm}$ for TM polarization. The propagation loss for TE polarization is estimated to be slightly higher, but comparable to the one reported for TM polarization. It is also important to mention that our measurement technique and analysis is sensitive to small shifts in the alignment of the input fiber with respect to the waveguide coupling structure. An approximate error of about $\pm 1 \mathrm{~dB}$ was therefore assumed in these measurements. An example plot illustrating the latter is shown in Fig. 5.10.

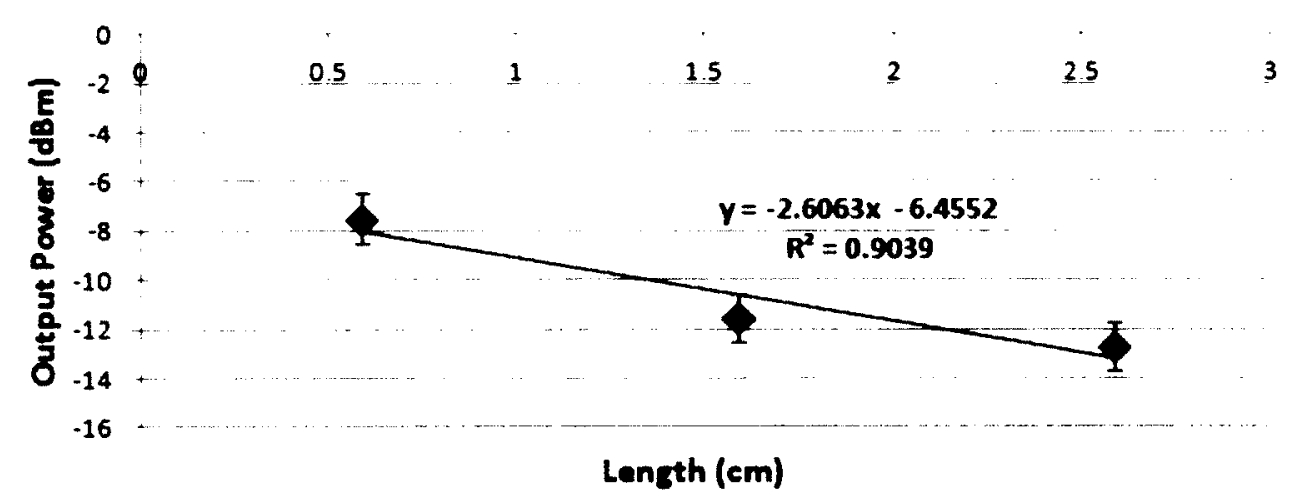

Figure 5.10 The power dependence on the length of an SWG (with uncertainties)

The output power dependence on the length of an SWG structure. The SWG structures had a nominal height, grating pitch, and grating duty cycle of $260 \mathrm{~nm}, 250 \mathrm{~nm}$, and $50 \%$. This particular structure was nominally $400 \mathrm{~nm}$ wide ( $3^{\text {rd }}$ sample). The propagation loss is found by calculating the slope of the linear fit (black solid line). The slope of this graph is $-2.6 \mathrm{~dB} / \mathrm{cm}$ and its correlation coefficient $\left(\mathrm{R}^{2}\right)$ is 0.9039 . The $\mathrm{y}$ error bars are 2 decibels long describing the uncertainty in the power reading.

\subsection{The Fabrication and Realization of Athermal BSWG Waveguides}

The mask layout of diverse BSWG structures is shown here. The steps for realizing BSWG athermal waveguides are explained in detail. The BSWG athermal waveguides will then be compared with the temperature-independent SWG waveguide of the previous section and the theoretical predictions. 


\subsubsection{BSWG Waveguide Mask Layout}

The layout for measuring the TO coefficient of the silicon-on-insulator BSWG waveguides is shown below. The author was responsible for designing the BSWG mask layout and for calculating their respective TO coefficients. Figure 5.11 is a screenshot of the bottom, center-bottom, and center-top of the mask layout. Fig. 5.12 is a screenshot of the top of the mask layout. 
(a)

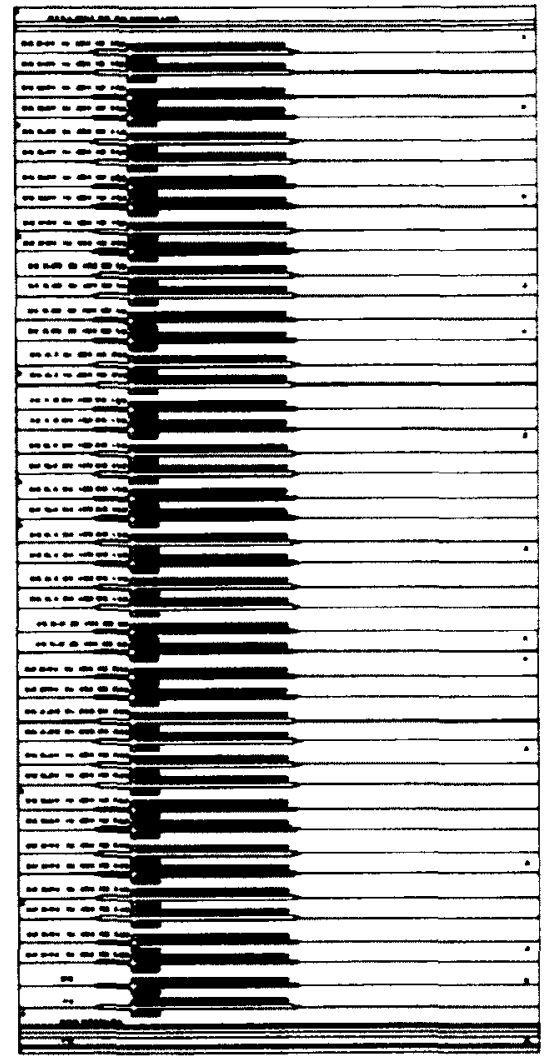

(b)

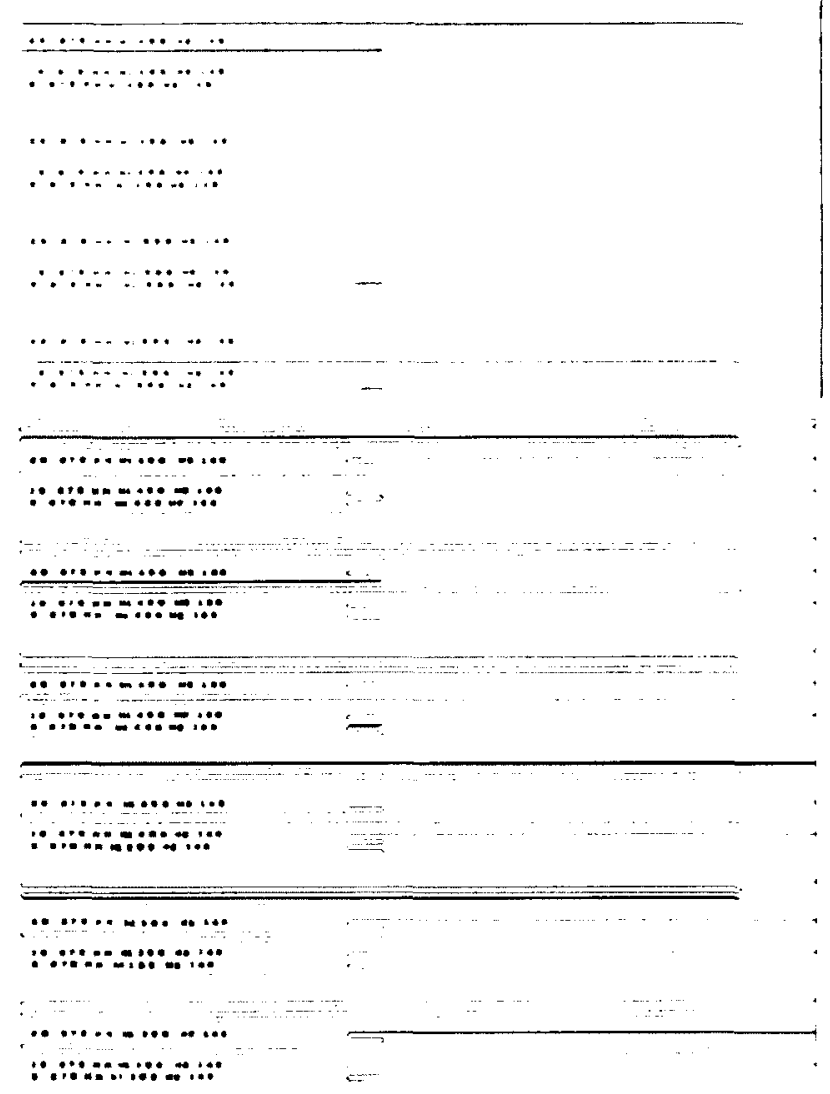

Figure 5.11 Screenshot of the BSWG mask layout (bottom and center)

A screenshot of the (a) bottom, (b) center-bottom, and (c) center-top part of the BSWG mask layout.

Various unbalanced Mach-Zehnder interferometers with varying grating duty cycles and bridge widths are illustrated in (a). SWG waveguides of different lengths, widths, and bridge widths for loss measurements are shown in (b) and (c). 


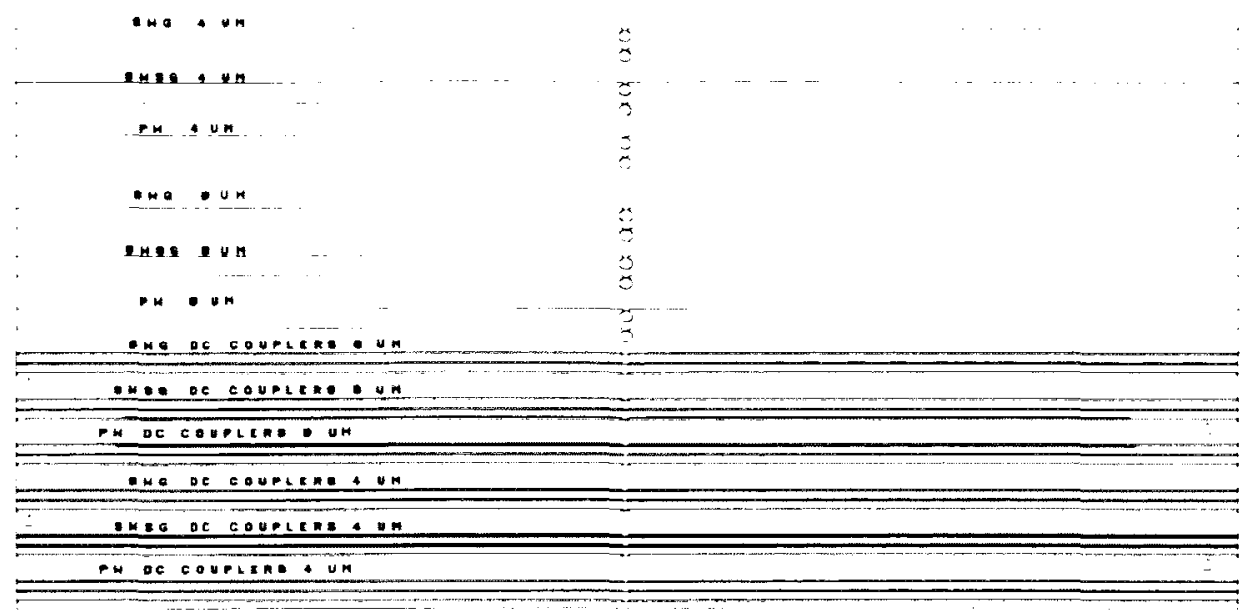

Figure 5.12 Screenshot of the top part of the BSWG mask layout

A screenshot of the top part of the mask layout. SWG and BSWG directional couplers and microring resonators are drawn here. Designing athermal SOI directional couplers and microring resonators using the SWG effect is possible.

To measure the effective TO coefficient of the BSWG waveguides, we followed the same methodology as reported in section 5.1.1. The $Y$ splitters and waveguide bends comprise photonic wire (PW) waveguides while each arm of the MZIs now comprises straight BSWG structures. The geometrical imbalance (length difference) between the two arms is the same as the one used for the SWG waveguide analysis $(\Delta \mathrm{L}=3 \mathrm{~mm})$. Coupling structures such as the one described in section 5.1.1 (Fig. 5.3) were used to adiabatically transform the PWs into BSWGs [50]. The PW-BSWG transformers are schematically shown in Fig. 5.14a, 5.14b, and 5.14c. BSWG structures of different lengths for measuring the propagation loss are also included in the layout (center-bottom and centertop part). Each loss structure has either a different waveguide width, $\mathrm{W}_{1}$, bridge width, $\mathrm{W}_{2}$, or both (see Fig. 5.13). The bends are comprised of PW waveguides for the same reasons mentioned in section 5.1.1. A close-up of several loss structures is shown in Fig. 
5.11. For a given waveguide and bridge width, the length of the structures is varied. The structures are $5.875 \mathrm{~mm}, 15.875 \mathrm{~mm}$, or $25.875 \mathrm{~mm}$ long.

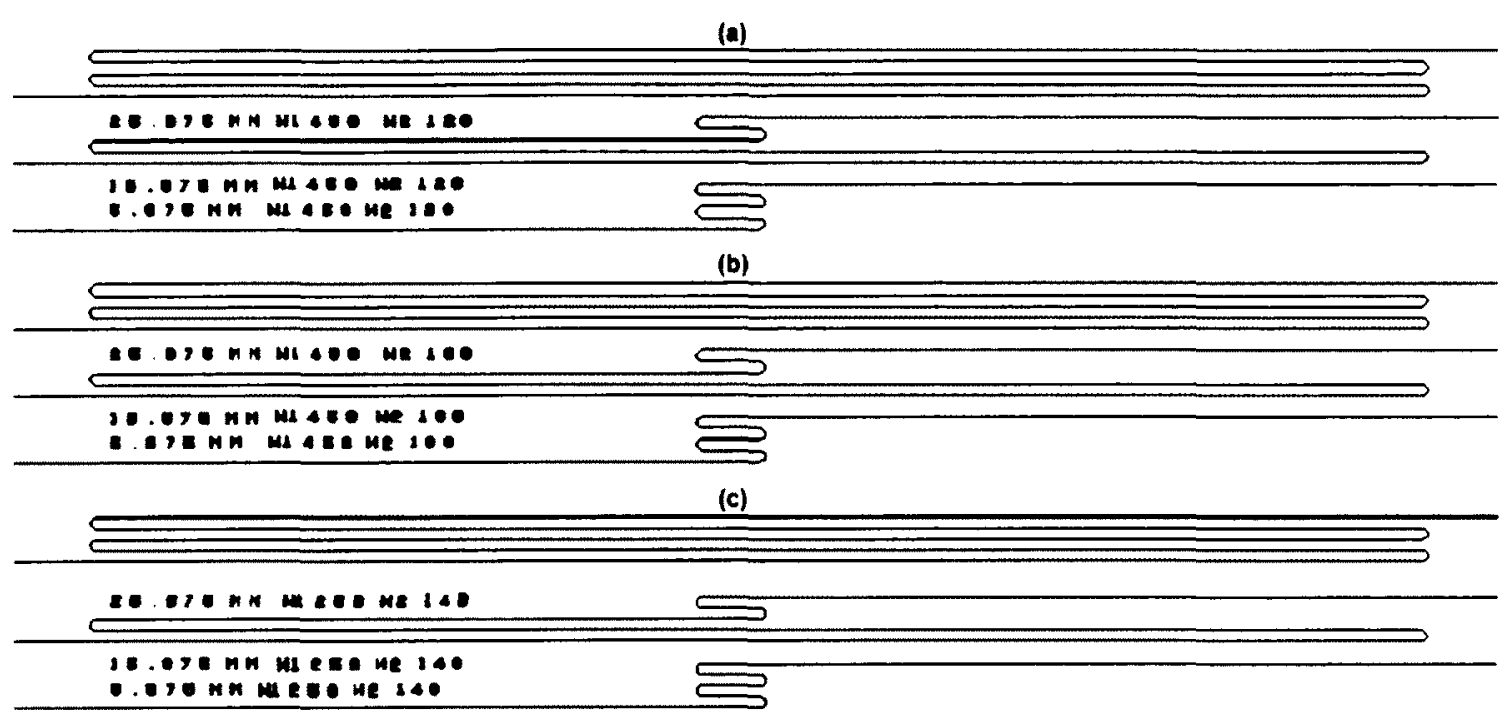

Figure 5.13 Close-up of different BSWG loss structures

A close-up of different loss structures with various lengths, waveguide widths, and bridge widths. The waveguides in (a) comprise BSWG structures with DC $=50 \%, W_{1}=450 \mathrm{~nm}$, and $W_{2}=120 \mathrm{~nm}$. The waveguides in (b) comprise BSWG structures with $\mathrm{DC}=50 \%, \mathrm{~W}_{1}=450 \mathrm{~nm}$, and $\mathrm{W}_{2}=100 \mathrm{~nm}$. The waveguides in (c) comprise BSWG structures with DC $=50 \%, W_{1}=250 \mathrm{~nm}$, and $W_{2}=140 \mathrm{~nm}$. 
(a)

(c)

Figure 5.14 Sections of the PW-BSWG mode transformers

The bridge width of the transformer is adiabatically reduced from its initial width $\left(\mathrm{W}_{1}\right)$ to the desirable BSWG bridge width $\left(\mathrm{W}_{2}\right)$. We clearly see that the bridge width from (a) to (c) is slowly changing. The desirable bridge width in this example is $140 \mathrm{~nm}$. The length of all PW-BSWG transformers is $10 \mu \mathrm{m}$.

\subsubsection{Fabrication}

The BSWG waveguides, like the SWGs, are silicon-on-insulator (SOI) waveguides [60]. The lower cladding layer is therefore silicon dioxide $\left(\mathrm{SiO}_{2}\right)$. The buried oxide layer is 2 $\mu \mathrm{m}$ thick and the BSWG silicon core segments are again $0.26 \mu \mathrm{m}$ thick as in our simulations.

The fabrication procedure was identical to the SWG waveguide samples described in the previous section. Please refer to section 5.1.2 for more details.

\subsubsection{Experimental Setup}

The experimental setup for measuring the thermo-optic coefficient of the BSWG structures is the same as the one described in section 5.1.3. The samples are mounted on an adjusting optical bench with a temperature-controlled copper heat sink. The TE or TM 
polarized light is coupled into the MZIs, loss structures, and rings via optical fiber. For a pictorial description of the setup, please refer to Fig. 5.5.

\subsubsection{Analysis and Procedure}

The methodology for measuring the TO coefficient of the BSWG waveguides that are in the sample is the same as the one described in section 5.1.4. The first step is to measure the optical transmission spectrum of the unbalanced MZIs at slightly different temperatures by placing the sample on the temperature-controlled heat sink. Figure 5.15 illustrates optical transmission of two MZI devices, one with BSWGs near athermal operation and the other with PWs, operating at different temperatures for TM polarization. The actual width, height and grating pitch of these waveguides are $490 \mathrm{~nm}$, $260 \mathrm{~nm}$, and $250 \mathrm{~nm}$, respectively.

As mentioned before, the effective thermo-optic (TO) coefficient of any waveguide (PWs, SWGs, or BSWGs) is measured from the temperature-induced wavelength shift of the unbalanced MZI spectrum as shown in Fig. 5.15 using equation (5.3) [61]. We notice, from the figure, that the temperature-induced wavelength shift is substantially smaller for the BSWG waveguide with a grating duty cycle and bridge width of $42 \%$ and $220 \mathrm{~nm}$, respectively. A sign reversal in the spectral shift is also observed from Fig. 5.15a to Fig. 5.15b. A temperature-induced shift of about $35 \mathrm{pm} /{ }^{\circ} \mathrm{C}$ and $-2.5 \mathrm{pm} /{ }^{\circ} \mathrm{C}$ is reported in (a) and (b) of Fig. 5.15. 
(a)

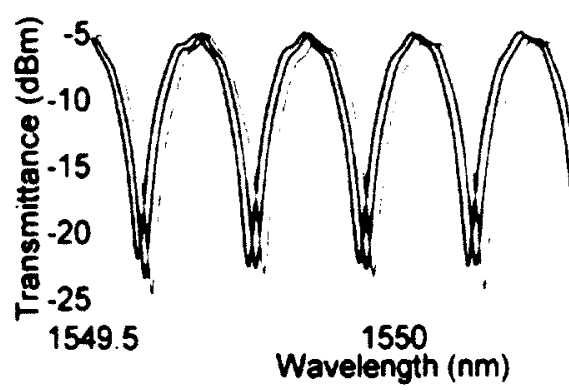

(b)

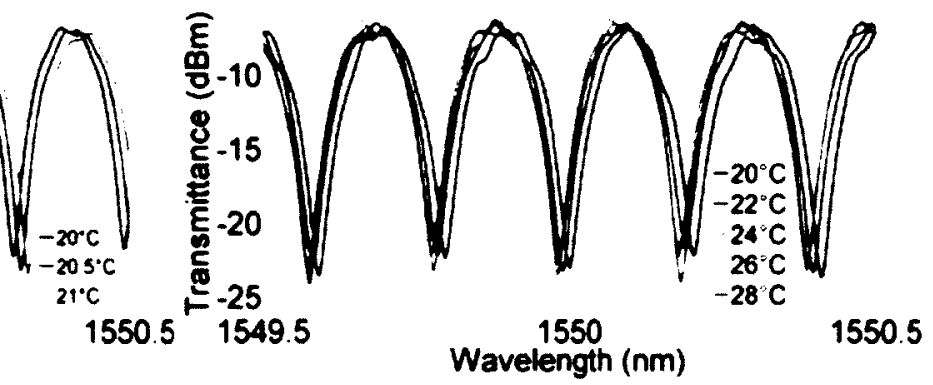

Figure 5.15 The spectral transmittance of the MZIs (PW and BSWG)

The spectral transmittance of MZI with (a) photonic wire waveguides and (b) near athermal BSWGs. A wavelength shift $\mathrm{d} \lambda / \mathrm{dT} \approx 35 \mathrm{pm} /{ }^{\circ} \mathrm{C}$ is observed in (a). $\mathrm{d} \lambda / \mathrm{dT} \approx-2.5 \mathrm{pm} /{ }^{\circ} \mathrm{C}$ is observed in (b). Waveguide dimensions are: (a) $W=490 \mathrm{~nm}$ and $\mathrm{H}=260 \mathrm{~nm}$; (b) $W_{1}=490 \mathrm{~nm}, \mathrm{H}=260 \mathrm{~nm}, \Lambda=250 \mathrm{~nm}, \mathrm{DC}=42 \%$, and $\mathrm{W}_{2}=220 \mathrm{~nm}$. The guided light is TM polarized

The calculations for measuring the TO coefficient from the shift at each minimum were carried out in MATLAB and are shown in appendix B. The results are illustrated in Fig. 5.16. The figure gives us insight on the variations of the TO coefficient per nanometer change in the operating wavelength, i.e. the measured BSWG waveguide "thermal" bandwidth (see section 4.2.4). The solid lines in the figure are linear fits to the data. 


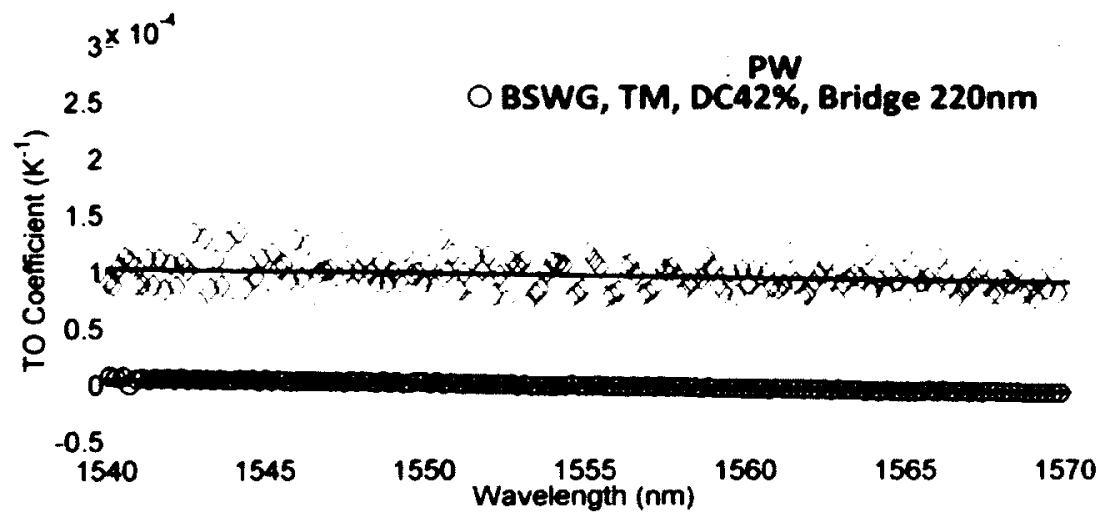

Figure 5.16 The TO coefficient dependence on the wavelength (TM, DC $=42 \%$ )

The TO coefficients of a quasi-athermal BSWG waveguide is compared to the TO coefficients of the PW waveguide. The BSWG waveguide is designed nominally athermal for TM polarization at $\lambda=1550 \mathrm{~nm}$. Its width, bridge width, height, grating pitch, and duty cycle are $490 \mathrm{~nm}, 220 \mathrm{~nm}, 260 \mathrm{~nm}, 250 \mathrm{~nm}$, and $42 \%$, respectively.

Since the bridge width $\mathrm{W}_{2}$ represents an additional degree of freedom in the design of temperature-independent waveguides compared to the SWG waveguides, there is more than one solution for designing such waveguides. According to the simulation results reported in section 4.2.3, athermal operation in BSWG waveguides can be achieved by judiciously choosing the bridge widths $\mathrm{W}_{2}$ for any grating duty cycle provided that the latter is not too small, nor large. In the example above, we see that BSWG waveguides with a grating duty cycle of $42 \%$ are temperature independent for a bridge width of $\sim 220$ $\mathrm{nm}$, for TM polarization. The waveguide shown in Fig 5.15 is near temperature independent over the entire measured wavelength range $(1540-1570 \mathrm{~nm})$, with a residual $\mathrm{dn}_{\text {eff }} / \mathrm{dT}$ from $5.0 \times 10^{-6} \mathrm{~K}^{-1}$ to $-6 \times 10^{-6} \mathrm{~K}^{-1}$, while $\mathrm{dn} / \mathrm{dT} \sim 0$ at the wavelength $\lambda=1554$ 
$\mathrm{nm}$. The measured change in the TO coefficient per $1 \mathrm{~nm}$ change in the operating wavelength is approximately $-3.7 \times 10^{-7} \mathrm{~K}^{-1} / \mathrm{nm}$.

Fig. 5.17a illustrates a similar near-athermal behavior for a BSWG waveguide with a grating duty cycle of $42 \%$ that is guided with TE polarized light. A bridge width of 200 $\mathrm{nm}$ is required in that case. Fig. $5.17 \mathrm{~b}$ shows a near-athermal behavior for a grating with $58 \%$ duty cycle and a bridge width of $140 \mathrm{~nm}$. The latter is quasi-athermal for TM polarization only. For TE polarization, the bridge width required is very small and it will hence be difficult to fabricate such a structure (see section 5.2.5). 


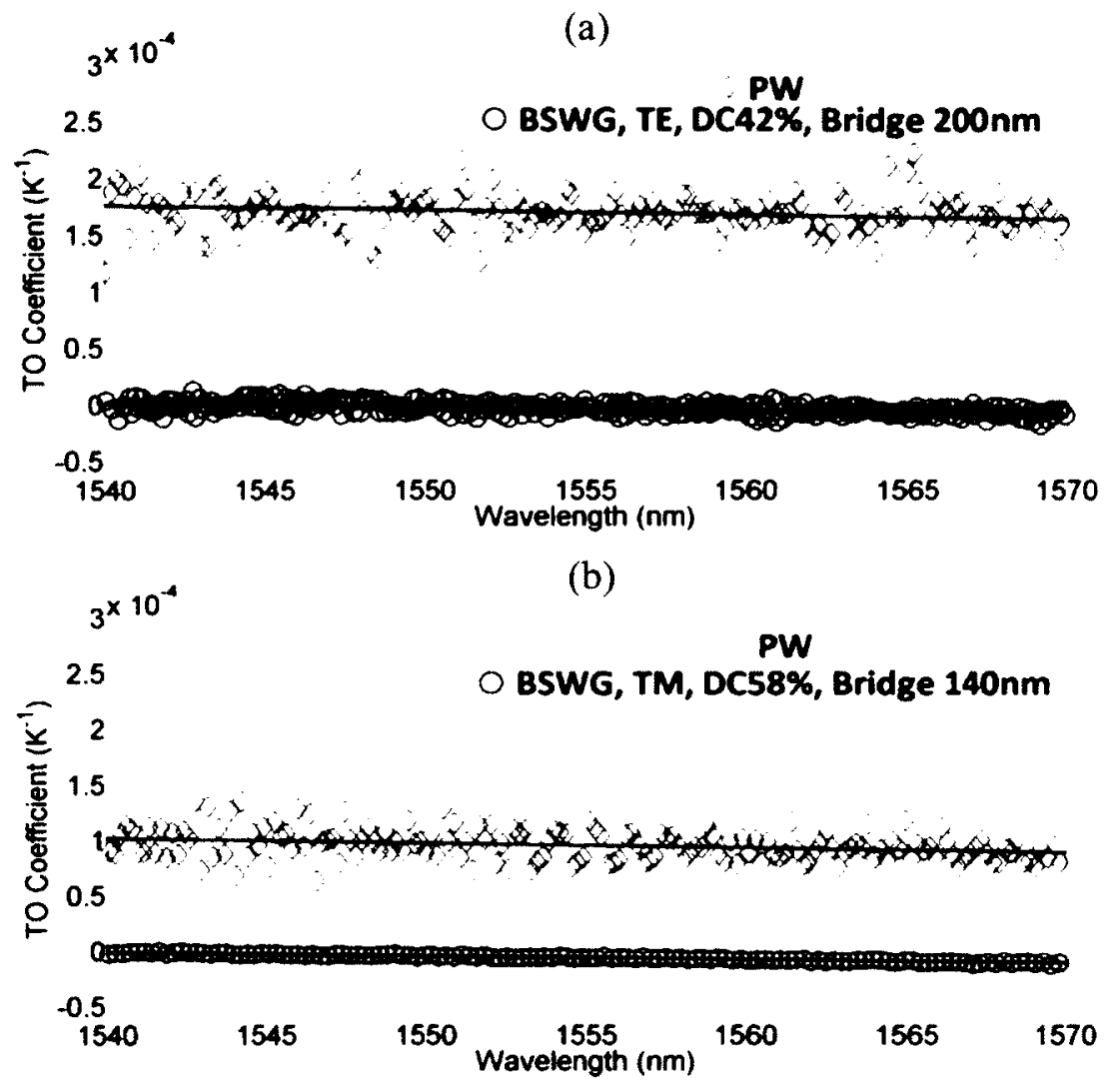

Figure 5.17 The TO coefficient dependence on the wavelength (other waveguides)

The TO coefficients of two other quasi-athermal BSWG waveguides are compared to the TO coefficients of the PW waveguide. The BSWG in (a) is quasi-athermal for TE polarization. Its width, bridge width, height, grating pitch, and duty cycle are $490 \mathrm{~nm}, 200 \mathrm{~nm}, 260 \mathrm{~nm}, 250 \mathrm{~nm}$, and $42 \%$, respectively. The BSWG in (b) is quasi-athermal for TM polarization. Its width, bridge width, height, grating pitch, and duty cycle are $490 \mathrm{~nm}, 140 \mathrm{~nm}, 260 \mathrm{~nm}, 250 \mathrm{~nm}$, and $58 \%$, respectively.

Due to the difficulties in fabricating such small waveguides, the actual dimensions deviated from the design by several tens of nanometers. For our analysis, it is crucial that we use the correct dimensions in our simulations. We used SEM images of the waveguides to determine the actual dimensions. In the analysis of our SEM images, we found a relatively large uncertainty in the dimensions measurements, so we had to use 
our best estimate of the dimensions for the simulations. The difficulties, i.e. the resolution of the SEM image and the determination of the precise structure outline are shown in Fig. 5.18. For example, the large white border line outlining the structure may or may not be part of the structure. We also carried out measurements of the TO coefficient of the SU-8 films using an ellipsometer with a heated sample stage and found a value of $1.3 \times 10^{-4} \mathrm{~K}^{-1}$, a value that is slightly larger than the one assumed previously from the literature. This adjusted value was used in the simulations.

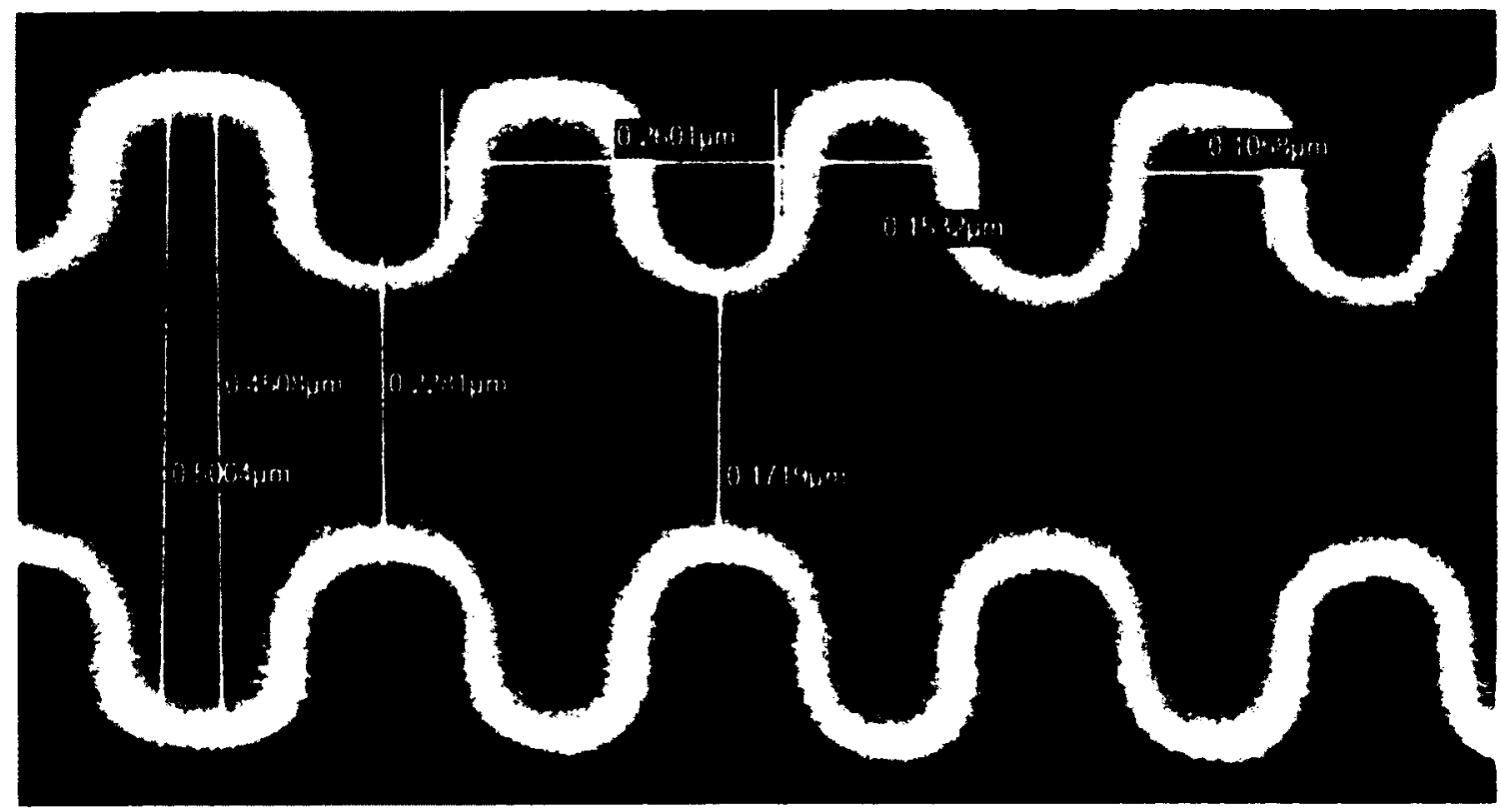

Figure 5.18 The uncertainties in the BSWG waveguide parameters

The uncertainties in the BSWG waveguide parameters are showcased. It is difficult to measure with precision the width $\left(\mathrm{W}_{1}\right)$, length $(a)$, bridge width $\left(\mathrm{W}_{2}\right)$, and grating pitch $(\Lambda)$. These are factors that can explain the small discrepancies between the theory (simulations) and experiment.

\subsubsection{Comparison with MPB Simulations}


The comparison with the experimental results is presented in Fig. 5.19, where the TO coefficients of BSWG waveguides with $42 \%$ and $58 \%$ grating duty cycles are shown for TE and TM polarizations as a function of bridge widths. Additional simulations with the actual waveguide dimensions of the fabricated BSWG waveguides were carried out in order to see how well the data matches with the theory. Fig. 5.19 includes the measured TO coefficient values of BSWG waveguides with duty cycles of $42 \%$ and $58 \%$. The BSWG waveguides with $\mathrm{DC}=42 \%$ have varying bridge widths of $180 \mathrm{~nm}, 200 \mathrm{~nm}, 220$ $\mathrm{nm}, 240 \mathrm{~nm}$, and $260 \mathrm{~nm}$. The BSWG waveguides with $\mathrm{DC}=58 \%$ have bridge widths of $140 \mathrm{~nm}, 160 \mathrm{~nm}, 205 \mathrm{~nm}$, and $265 \mathrm{~nm}$.
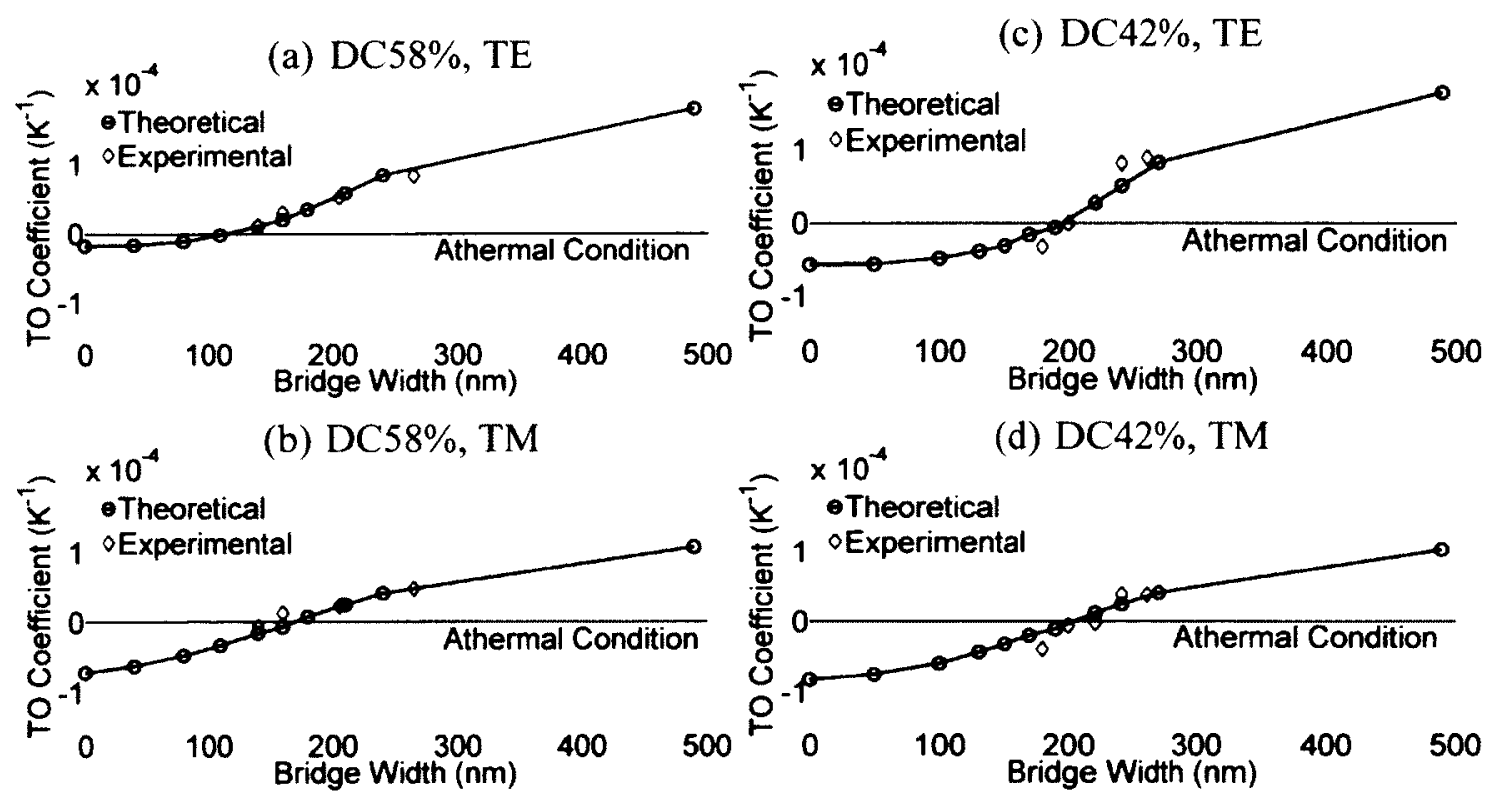

Figure 5.19 The BSWG TO coefficient dependence on the bridge width

BSWG thermo-optic coefficient dependence on the bridge width $\left(\mathrm{W}_{2}\right)$, for $\mathrm{DC}=58 \%(\mathrm{a}, \mathrm{b})$ and $\mathrm{DC}=42 \%$ (c, d) for TE and TM polarizations. Simulation results for waveguide dimensions measured by SEM are compared with the experimental data. Waveguide dimensions: $W_{1}=490 \mathrm{~nm}, H=260 \mathrm{~nm}$, and $\Lambda=250 \mathrm{~nm}$. The operating wavelength is $1550 \mathrm{~nm}$. 
The results in Fig. 5.19 show that the experiments agree well with the theoretical predictions. Some minor differences are most likely due to uncertainties in the SEM measurement of the actual waveguide dimensions as shown in Fig. 5.18 in section 5.2.4. It should also be noticed that the critical dimensions of the athermal BSWG waveguides demonstrated here are larger than $100 \mathrm{~nm}$ and can therefore potentially be fabricated using deep-UV lithography, a cheaper alternative to electron beam lithography. It is worth mentioning that electron beam lithography is rather expensive and is therefore not recommended for mass production.

\subsubsection{BSWG Loss Measurements}

Investigations on the propagation loss of the BSWG structures have been carried out as well. The procedure for measuring the propagation loss of the BSWG structures is the same as described in section 5.1.6. We have encountered several difficulties due to the couplers and PW-BSWG mode transformers. In most cases, given a specific waveguide and bridge width ( $\mathrm{W}_{1}$ and $\mathrm{W}_{2}$, respectively), we were only able to measure a single data point which corresponds to the output power of the shortest structure ( $\mathrm{L}=5.875 \mathrm{~mm}$ ). Complications such as reflections, scattering, and inefficient coupling were overly dominant especially for TE polarization. We have been able to collect two or three data points in some cases (the output power of the loss structures where $\mathrm{L}=5.875 \mathrm{~mm}$, and $\mathrm{L}$ $=15.875 \mathrm{~mm}$ ), but only for TM polarization. The propagation loss for TM polarization of BSWG waveguides with varying waveguide and bridge widths ( $W_{1}$ and $W_{2}$, respectively) is tabulated below (see tables 5.2 and 5.3). The data presented below is however not very accurate due to alignment uncertainties (see section 5.1.6) and lack of data, but gives us a 
rough estimate of the propagation loss. According to tables 5.2 and 5.3, the BSWG propagation loss for TM polarization appears to be greater, but comparable to the loss of PW and SWG waveguides. The BSWG structures here had a nominal height, grating pitch, and grating duty cycle of $260 \mathrm{~nm}, 250 \mathrm{~nm}$, and $50 \%$.

\begin{tabular}{|c|c|}
\hline BSWG Waveguide Width, $\mathbf{W}_{\mathbf{1}} \mathbf{( n m )}$ & Propagation Loss $(\mathbf{T M})(\mathbf{d B} / \mathbf{c m})$ \\
\hline 250 & 8.6 \\
\hline 300 & 5.9 \\
\hline 350 & 6.4 \\
\hline 400 & 9.8 \\
\hline 450 & 7.3 \\
\hline 550 & 6.2 \\
\hline
\end{tabular}

Table 5.2 The propagation loss as a function of the BSWG waveguide width (TM)

The propagation loss for TM polarization as a function of the BSWG waveguide width $\left(W_{1}\right)$. The bridge width of the waveguides here is $140 \mathrm{~nm}$. In some cases, we lacked data points due to inconsistencies and malfunctioning of the passive loss devices. The propagation loss resulting from that data is therefore not very accurate. Yet, we conclude that the BSWG propagation loss is slightly greater than the SWG propagation loss $(\sim 7.4 \mathrm{~dB})$.

\begin{tabular}{|c|c|}
\hline BSWG Bridge Width, $\mathbf{W}_{\mathbf{2}}(\mathbf{n m})$ & Propagation Loss $(\mathbf{T M})(\mathrm{dB} / \mathbf{c m})$ \\
\hline 100 & 9.1 \\
\hline 120 & 10.3 \\
\hline 140 & 7.3 \\
\hline 160 & 6.9 \\
\hline
\end{tabular}

Table 5.3 The propagation loss as a function of the BSWG bridge width (TM)

The propagation loss for TM polarization as a function of the BSWG bridge width $\left(\mathrm{W}_{2}\right)$. The nominal width of the waveguides here is $450 \mathrm{~nm}$. In some cases, we lacked data points due to inconsistencies and malfunctioning of the passive loss devices. The propagation loss resulting from that data is therefore not

very accurate. Yet, we conclude that the BSWG propagation loss is slightly greater than the SWG propagation loss $(\sim 8.4 \mathrm{~dB})$. It also seems that the propagation loss increases with decreasing bridge width. 


\section{Chapter: Conclusion and Future Work}

\subsection{Summary}

In conclusion, we first explained the importance of designing components and devices that are insensitive to temperature changes. Slight temperature changes can induce undesirable effects that will hinder the device performance. A precise control of temperature is consequently very important in many applications. A brief history of past work on athermalization of waveguide devices was then summarized.

We thoroughly studied the subwavelength grating effect. The SWG effect allowed us to engineer artificial effective materials with optical effective indices $\mathrm{n}_{\mathrm{eff}}$. By doing so, we gained further control in the adjustment of the material's refractive index. The resulting effective index was hence determined by the grating geometry of the comprising materials. The subwavelength technique circumvented limitations on both temperature instability and refractive index constraints due to the material platform.

We then demonstrated, both numerically and experimentally, that the subwavelength grating spatial averaging effect can be used to make temperature-independent waveguides with a composite core consisting of silicon and SU-8 polymer, compatible with standard silicon photonic wire waveguides. Athermal behavior was achieved for both TE and TM polarizations, albeit for different SWG duty ratios. Our calculations, for example, show that athermal operation will occur for a grating duty cycle of $57 \%$ for TE and $82 \%$ for TM for a standard photonic wire cross section $(260 \times 450 \mathrm{~nm}, \Lambda=250 \mathrm{~nm})$. These results 
on engineering the TO waveguide properties with subwavelength gratings are thought to be a significant step forward toward developing temperature-insensitive silicon photonic circuits.

Yet, despite the advantages that the SWG waveguides offer, we found that there is room for improvement as the realization of athermal SWG waveguides for TM polarized light remains difficult due to the waveguide's size constraints. We therefore embedded bridge segments in the grating to reduce the grating duty cycle and enlarge the gaps between the subsequent silicon core segments. In other words, by bridging the core silicon segments with narrower segments, the latter geometry circumvented the technological limitation that prevented fabrication of athermal SWG waveguides for the TM mode, i.e. large grating duty cycles of more than $80 \%$ were avoided. The temperature-independent BSWG waveguides were consequently shown to have relaxed fabrication requirements.

The temperature-independent bridge subwavelength grating waveguides were demonstrated for both TE and TM polarizations. Compared to conventional segmented SWG waveguides, the bridging segments provided an extra degree of freedom in the design of the BSWG waveguides, lending to several possible geometries of athermal BSWG waveguides. For a given range of duty cycles, an athermal condition for both TE and TM polarizations was achieved by a judicious choice of the bridge width. Temperature-independent BSWG waveguides with two different grating duty cycles were moreover demonstrated experimentally. The propagation losses of the BSWG waveguides were however measured to be larger, but comparable to the propagation 
losses of the standard photonic wires and SWGs. We therefore conclude the viability of temperature-independent BSWG waveguides as alternate solutions to SWG and photonic wire waveguides.

\subsection{Future Work}

While all the results discussed in this paper are for silicon-SU-8 polymer SWG-BSWG waveguides, other polymers could have been used to compensate for silicon's positive TO coefficient. As mentioned in Chapter 2, silicon's positive TO coefficient will be compensated as long as the core's other composite material, i.e. the cladding material, has a negative TO coefficient. An SWG waveguide comprising a polymer material with a highly negative TO coefficient would require higher grating duty cycles in order to achieve athermal operation and vice versa. A different choice of polymer can therefore improve the temperature sensitivity of the quasi-athermal SWG waveguides. A polymer with a small negative TO coefficient for example can be an interesting choice for the TM mode especially. The grating duty cycle would not have to be as high.

Moreover, one could look at further applications of SWGs and BSWGs as they provide significant advantages. Applications include the realization of athermal SWG and BSWG microring resonators, directional couplers, MZIs, and AWGs. SWG and BSWG structures, as demonstrated, provide more freedom in choosing the refractive-index contrast between the core and cladding. In other words, the effective index of the composite core can be somewhat adjusted. Control of both temperature and optical properties simultaneously can therefore become realizable. Research on the design and 
fabrication of temperature-independent SWG and BSWG directional couplers and microring resonators is ongoing.

\subsection{Achievements and Awards}

During the completion of this project, I received numerous awards and published several papers. First of all, I managed to publish three conference papers, one journal paper, and was also listed in many co-authored journal publications. The following is a list of all the papers in my name:

1. Marc Ibrahim, Jens H. Schmid, Alireza Aleali, Pavel Cheben, Jean Lapointe, Siegfried Janz, Przemek J. Bock, Adam Densmore, Boris Lamontagne, Rubin Ma, Dan-Xia Xu, and Winnie N. Ye, "Athermal silicon waveguides with bridged subwavelength gratings for TE and TM polarizations", Optics Express 20 (16) 18356-18361 (2012)”

2. M. Ibrahim, A. Aleali, J.H. Schmid, P.Cheben, J. Lapointe, S. Janz, P.J. Bock, A. Densmore, B. Lamontagne, R. Ma, D.-X. Xu, and W.N. Ye, “Temperatureindependent silicon waveguides comprising bridged subwavelength gratings", Proc. SPIE 8412, Photonics North 2012

3. Ibrahim, M., Schmid, J.H., Cheben, P., Lapointe, J., Janz, S., Bock, P.J., Densmore, A., Lamontagne, B., Ma, R., Dan-Xia Xu, Ye, W.N., “Athermal silicon subwavelength grating waveguides", Group IV Photonics 258-260 (2011) 
4. M. Ibrahim, J.H. Schmid, P.Cheben, J. Lapointe, S. Janz, P.J. Bock, A. Densmore, B. Lamontagne, R. Ma, D.-X. Xu, and W.N. Ye, "Athermal silicon subwavelength grating waveguides", Proc. SPIE, Photonics North 2011

5. P. Cheben, J. H. Schmid, P. J. Bock, J. Lapointe, S. Janz, D.-X. Xu, A. Delâge, M. Vachon, R. Halir, A. Ortega-Monux, C. Alonso Ramos, I. Molina-Fernandez, J.-M. Fédéli, M. Ibrahim, W. N. Ye, A. V. Velasco, M. L. Calvo, and I. Glesk, "Refractive Index Engineering with Sub-Wavelength Gratings in Silicon Waveguides", Advanced Photonics Congress IPRSN, OSA, (2012)

6. Yule Xiong, Marc Ibrahim, and Winnie N. Ye, Impact of LOCOS techniques on photonic wire waveguides, Applied Optics 51 (29) 7089-7093 (2012)

7. J. H. Schmid, M. Ibrahim, P. Cheben, J. Lapointe, S. Janz, P. J. Bock, A. Densmore, B. Lamontagne, R. Ma, W. N. Ye, and D.-X. Xu, "Temperatureindependent silicon subwavelength grating waveguides', Optics Express 36 (11) $2110-2112(2011)$

Moreover, I received scholarships and awards for the progress of this project. In 2010, I received the Ed Ireland Award for Engineering valued at $\$ 500$. In 2011, I was granted a scholarship from The Natural Sciences and Engineering Research Council of Canada (NSERC) valued at around $\$ 18,000$.

At last, I had the privilege of going to numerous conferences and enrich my knowledge. I have not only published conference papers but have also presented my project to many other students and professionals. I gave three poster presentations: one at the 2011 
Photonics North conference, one at the 2011 Group IV Photonics conference held in London, England, and the other at the Canadian Institute for Photonic Innovations (CIPI) Annual General Meeting. I won the $2^{\text {nd }}$ prize for my poster presentation at the latter event. 


\section{Appendices}

Appendix A

MPB Basics

\section{A.1 MPB Script and Terminology-SWG Waveguide Structure}

This software package, like any other package, has its own terminology and syntax. Here, we will focus on the terminology that was used in the MPB scripts during the analysis. For a complete list of all the terms, please refer to [55].

The following is an example of a written MPB script describing the structure of an SWG waveguide. As we can see, all commands are written inside brackets and the semicolon marks the start of a comment. The script only describes the contents of a unit cell, i.e. one segment of the SWG. 


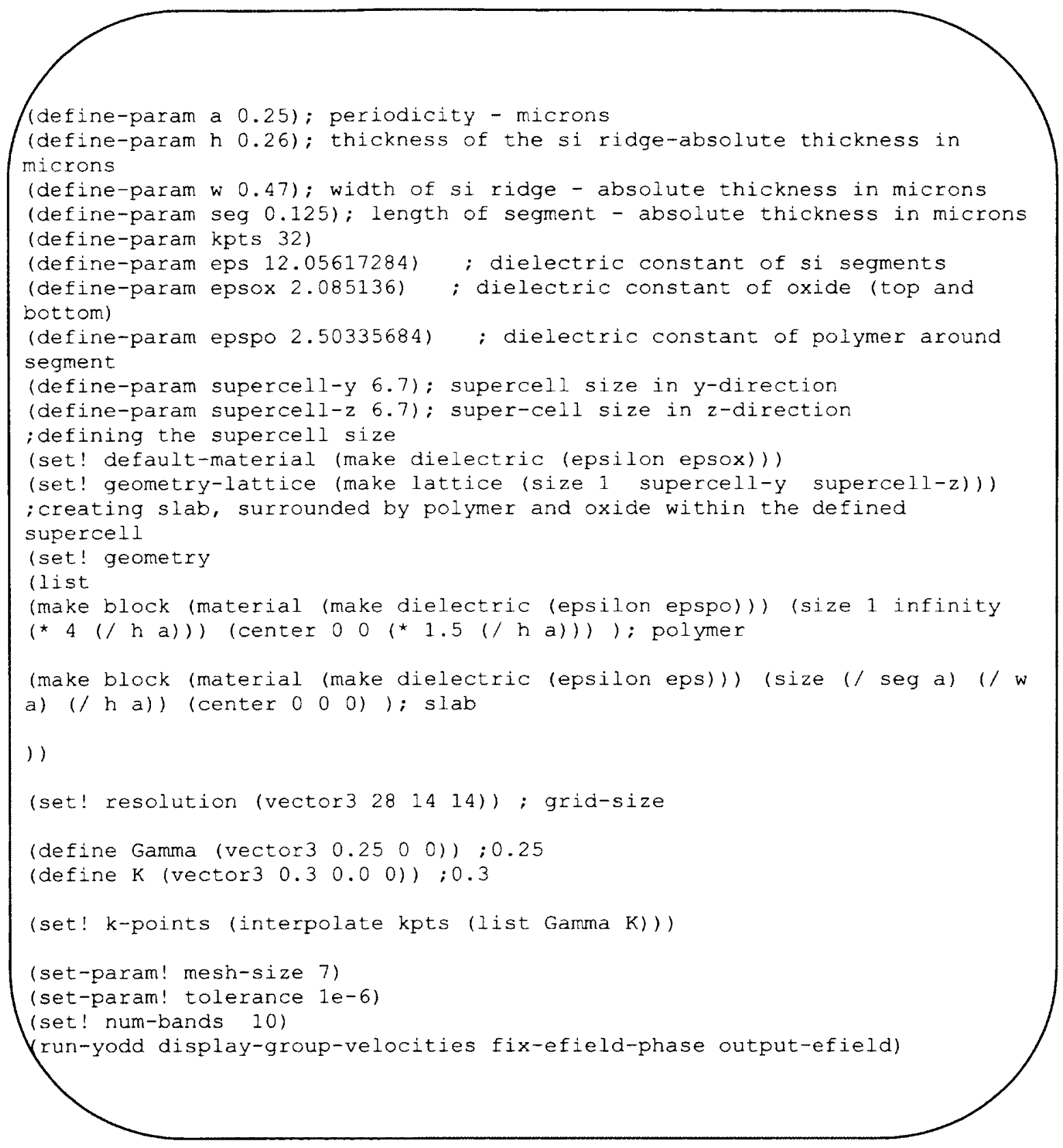

First of all, in that MPB script, the waveguide parameters are being declared with the define-param command. These include the dimensions of the waveguide, the dielectric constants, the number of k-points, etc. The parameters can have any given name provided that it is being used repeatedly throughout the script. Yet, the MPB software also has its own set of parameters which include the geometry of the structure 
(geometry), the resolution of the grid (resolution), the grid's mesh size (meshsize), and the number of photonic bands computed at each k-point (num-bands) amongst others. A more detailed explanation of the significance of the MPB parameters can be found in [55]. We must remember that all of these parameters have a default setting and therefore, we simply need to specify the ones we desire to change. To change the default setting of a parameter, we must use the set! command. For example, we had to change the "background" material (substrate) with silicon dioxide (epsox). We did that in the following manner:

(set! default-material (make dielectric (epsilon epsox)))

Moreover, to define the blocks contained in our unit cell, we have used the make block command. On top of the substrate epsox, we have constructed a three-dimensional block of polymer epspo. A smaller sized three-dimensional block of silicon corresponding to one core segment of the SWG was then put on top of the polymer block. Here, only one cell was described as these blocks are reproduced periodically in all three directions creating our desired SWG structure. The make block commands are shown below.

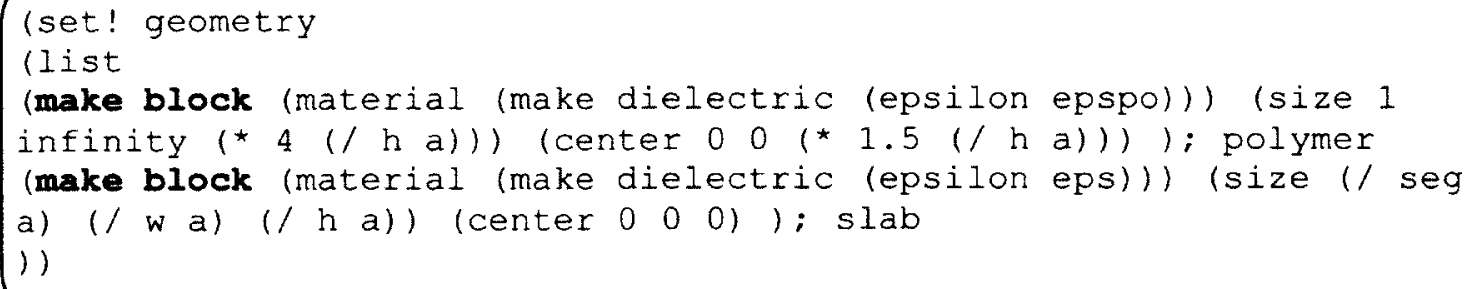


As we can see from the latter segment of MPB code, the blocks were positioned such that we obtain our structure. Yet, the mathematical syntax used for describing the size, position, and orientation of the blocks may not be intuitive for less experienced readers. We will hence take a few moments to explain it in the next subsection. A discussion on units will follow.

Finally, once we are done defining the structure and setting the parameters to their respective values, we are now ready to run the program. The MPB program can give solutions for both the TE and TM modes. When running the program, we had to specify the polarization of the light for the desired solutions. To look at the TE modes along with their group velocities and their electric field distribution, the following command had to be entered:

(run-yodd display-group-velocities fix-efield-phase output-efield)

To look at the TM modes, at their group velocities, and their electric field distribution, a similar command had to be entered:

(run-yeven display-group-velocities fix-efield-phase output-efield)

Here, display-group-velocities, fix-efield-phase, and output-efield are output functions. There are many other output functions that we can pass onto the 
program. These are described in the user reference section of [55]. For more details, please consult the user reference section.

Before moving on to the next section, it is important to note that the SWG waveguide, which is essentially a one-dimensional grating, is periodic along one direction only (the $\mathrm{x}$-direction). Consequently, the periodic segments generated along the $\mathrm{y}$ - and $\mathrm{z}$-directions by the MPB software were disregarded by making the computation window large enough (see the size of supercell-y and supercell-z). The computational window was about seven times larger in the $y$ - and z-directions.

\section{A.2 MPB Mathematical Operations and Units}

The MPB software uses the common ordinary arithmetic operators and functions [55]. The notation however is different. The MPB software uses the "prefix" notation or the "Polish" notation to perform the mathematical operations. The mathematical operator, in this notation, precedes the operands that are necessary for a given operation. Therefore, if we wish to perform a multiplication of two numbers, say 3 and 5, we would have to write the operation in the MPB script in the following manner:

\section{$\star 35$}

In our previous example, mathematical operations were performed to create blocks within the unit cell. The size (size) and location (center) of these blocks was specified there. They depended on the length, width, height and the SWG pitch. For more details, please 
consult the operations following the make block commands in the MPB script example shown above.

Moreover, the MPB software package, in principle, offers freedom in choosing units to describe diverse geometries and materials [55]. Yet, the size of the lattice basis, for example, has to be adjusted accordingly for consistency. This is possible due to Maxwell's equations being scale invariant. In other words, if we already have a set of solutions for a specific set of sizes and the sizes are increased by a factor of 5 later on, the right set of solutions are simply the same ones multiplied by that same factor 5 . Therefore, if the situation arises, a problem only needs to be solved once. The solutions to that problem will be applied to all length scales.

As a result, we wrote all distances, coordinates, and dimensions in terms of the grating's periodicity (lattice constant). The size of the pitch (a), which is $250 \mathrm{~nm}$ in our example, is here considered to be unity. The distances are thus written in terms of a using the values assigned to the define-param parameters. For example, here we wanted, according to the MPB script, the length of the silicon block to be half the size of the grating pitch specifically:

(define-param a 0.25); periodicity - microns

(define-param $\mathrm{h} 0.26$ ); thickness of the si ridge-absolute thickness in microns

(define-param w 0.47); width of si ridge - absolute thickness in microns

(define-param seg 0.125 ); length of segment - absolute thickness in microns 
Hence, using the make block command, we have constructed, within the cell, a block made of silicon (eps) that is 0.5 units long ( $\mathrm{seg} / \mathrm{a}$, or in "Polish" notation, / seg a) in the $x$-direction. We must remember that everything here is written in terms of a.

(make block (material (make dielectric (epsilon eps))) (size (/ seg a) (/ w (a) (/ $\mathrm{h}$ a)) (center 000$)$ ): slab

However, the frequency eigenvalues and k-points obtained from simulations can't be expressed in terms of a fundamental lengthscale a [55]. The k-points are expressed in terms of the reciprocal lattice vectors as oppose to the unit-normalized lattice vectors. Therefore, if the k-points have dimensions no-size, the reciprocal lattice vectors are taken to be written in terms of $2 \pi / a$. On the other hand, the frequencies returned by the MPB program are in units of $\mathrm{c} / \mathrm{a}$, where $\mathrm{c}$ is the speed of light in vacuum and $a$ is the fundamental unit of lengthscale. Here, $a$ corresponds to the grating pitch. As a result, adjustments had to be made in order to find the actual frequency eigenvalues and k-points of the solutions to the photonic bands problem. The relationships between the normalized frequencies and $k$-points $\left(f_{\text {norm }}, k_{\text {norm }}\right)$, and their actual values $(f, k)$ are shown in equations (3.1) and (3.2).

$$
\begin{aligned}
& f=f_{\text {norm }} * \frac{c}{a} \\
& k=k_{\text {nerm }} * \frac{2 \pi}{a}
\end{aligned}
$$


In optics, wavelengths are rather used instead of frequencies to describe light. The relationship between the frequency and wavelength is a simple one:

$$
\lambda=\frac{c}{f}
$$

These relationships will come in handy when asked to calculate the effective index from the frequencies and k-points that will be given by the MPB program. Chapter 4 will cover that procedure.

\section{A.3 MPB File - SWG Waveguide Structure}

The following MPB file contains the telecommunications frequencies (around $f=193.55$ $\mathrm{THz}$ ) of the TE fundamental mode that is propagating inside an SWG structure with $\mathrm{W}=$ $450 \mathrm{~nm}, \mathrm{H}=260 \mathrm{~nm}, \Lambda=250 \mathrm{~nm}$, and $\mathrm{DC}=62 \%$. Their respective wavenumber $(\mathrm{k})$ is shown there as well. The waveguide is operating at room temperature. The fundamental k-band is defined here as "yodd band 1" and the frequencies as "yoddfreqs". The term "yodd" here refers to TE polarization. 32 normalized k-points $\left(\mathrm{k}_{\text {norm }}\right)$ ranging from 0.25 to 0.35 are shown in the file below. The normalized frequency $\left(f_{\text {norm }}\right)$ corresponding to the k-points is found in the last column. Please refer to Chapter 3 and equations (A.1) and (A.2) for further explanations on how to calculate the effective index from the normalized frequency and wavenumber. For more details, refer to section 4.1.1. 
yoddfreqs:, $k$ index, $k 1, k 2, k 3, \mathrm{kmag} / 2 \mathrm{pi}$, yodd band 1 , yoddfreqs:, $1,0.25,0,0,0.25,0.14129$,

yoddfreqs: $, 2,0.253226,0,0,0.253226,0.142497$,

yoddfreqs: $3,0.256452,0,0,0.256452,0.143689$, yoddfreqs: $4,0.259677,0,0,0.259677,0.144866$, yoddfreqs: $, 5,0.262903,0,0,0.262903,0.14603$, yoddfreqs:, $6,0.266129,0,0,0.266129,0.14718$, yoddfreqs: $, 7,0.269355,0,0,0.269355,0.148318$, yoddfreqs:, $8,0.272581,0,0,0.272581,0.149444$, yoddfreqs:, $9,0.275806,0,0,0.275806,0.150558$, yoddfreqs: $10,0.279032,0,0,0.279032,0.151663$, yoddfreqs:, 11, $0.282258,0,0,0.282258,0.152757$, yoddfreqs:, 12, $0.285484,0,0,0.285484,0.153842$, yoddfreqs:, 13, 0.28871, 0, 0, 0.28871, 0.154917, yoddfreqs:, 14, $0.291935,0,0,0.291935,0.155984$, yoddfreqs:, 15, 0.295161, 0, 0, 0.295161, 0.157043, yoddfreqs:, 16, 0.298387, 0, $0,0.298387,0.158093$, yoddfreqs: $17,0.301613,0,0,0.301613,0.159136$, yodafreqs:, 18, $0.304839,0,0,0.304839,0.160172$, yoddfreqs:, 19, $0.308065,0,0,0.308065,0.161201$, yoddfreqs:, 20, $0.31129,0,0,0.31129,0.162222$, yoddfreqs:, 21, $0.314516,0,0,0.314516,0.163238$, yoddfreqs: $22,0.317742,0,0,0.317742,0.164246$, yoddfreqs: $23,0.320968,0,0,0.320968,0.165249$, yoddfreqs:, 24, $0.324194,0,0,0.324194,0.166245$, yoddfreqs: , 25, $0.327419,0,0,0.327419,0.167235$, yoddfreqs:, 26, $0.330645,0,0,0.330645,0.16822$, yoddfreqs: $, 27,0.333871,0,0,0.333871,0.169199$, yoddfreqs:, 28, 0.337097, 0, 0, 0.337097, 0.170171, yoddfreqs:, 29, $0.340323,0,0,0.340323,0.171139$, yoddfreqs: $, 30,0.343548,0,0,0.343548,0.1721$, yoddfreqs:, $31,0.346774,0,0,0.346774,0.173056$, yoddfreqs:, $32,0.35,0,0,0.35,0.174007$,

\section{A.4 MPB Script - BSWG Waveguide Structure}




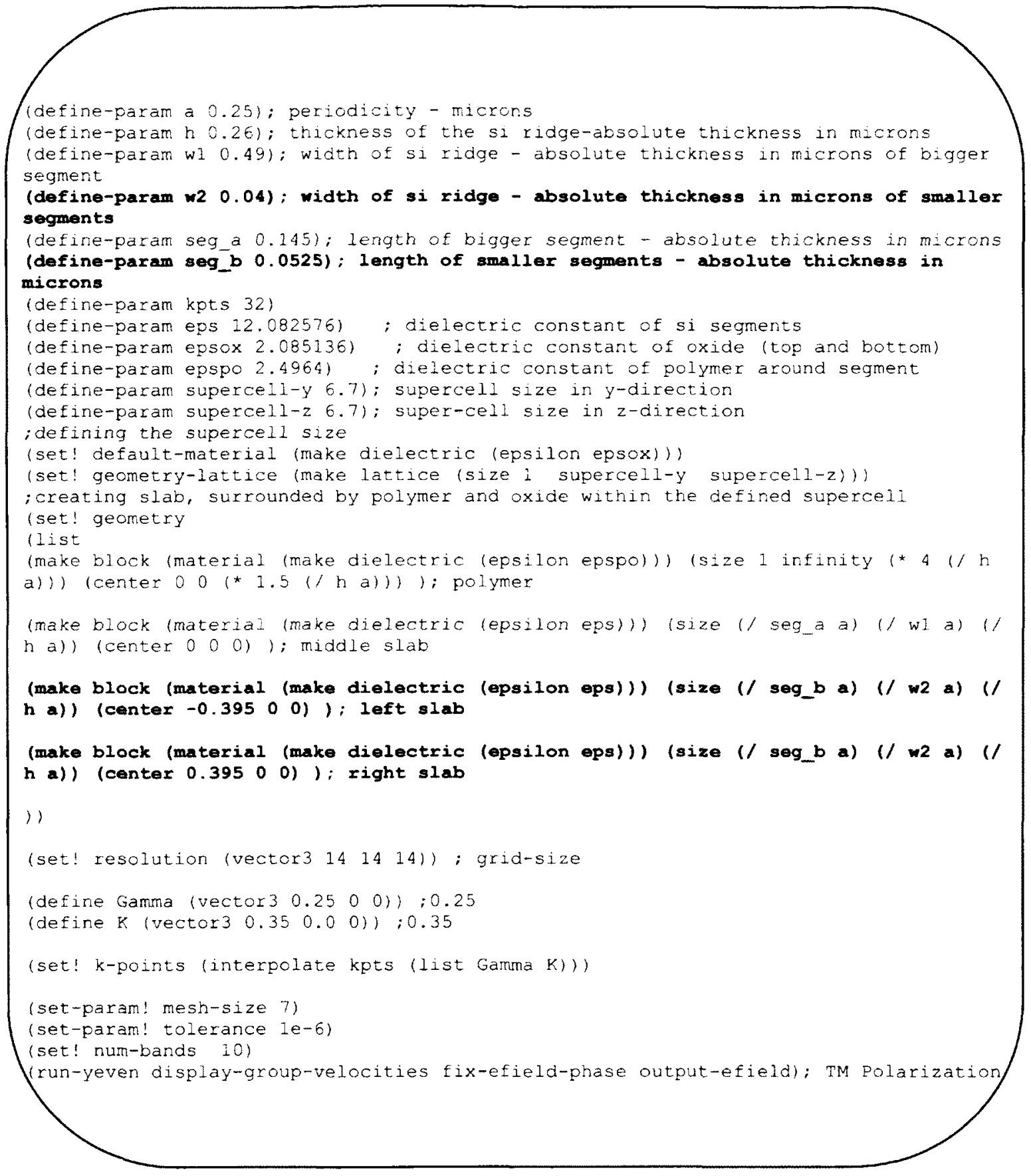

The MPB script shown above describes the waveguide structure of an SWG waveguide.

For further details, please refer to sections 4.2.1 and A.1. 


\section{A.5 MPB File - BSWG Waveguide Structure}

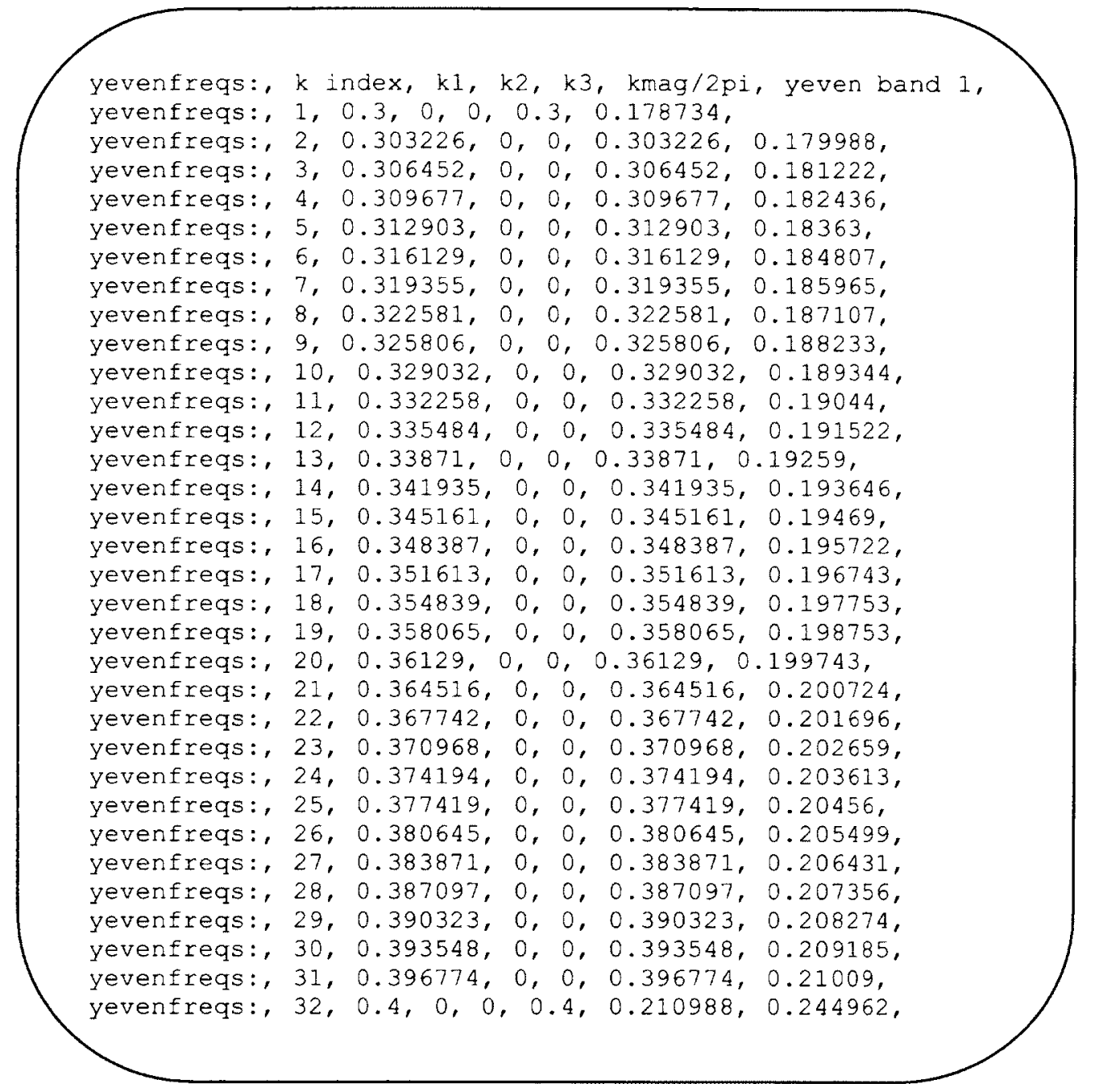

The MPB file, as shown above, generating the normalized frequencies and k-points around $\lambda=1550 \mathrm{~nm}(\mathrm{f}=193.55 \mathrm{THz})$ are similar to the one that was generated for the SWG structure (see sections 4.1.1 and A.3). The analysis thereon is essentially the same. The MPB file comprising the frequencies $\left(f_{\text {norm }}\right)$ and wavenumbers $\left(k_{\text {norm }}\right)$ of the TM fundamental mode propagating inside a BSWG structure with $\mathrm{W}_{1}=450 \mathrm{~nm}, \mathrm{~W}_{2}=140$ 
$\mathrm{nm}, \mathrm{H}=260 \mathrm{~nm}, \Lambda=300 \mathrm{~nm}$, and $\mathrm{DC}=50 \%$ at $\mathrm{T}=293 \mathrm{~K}$ is shown below. The term "yeven" stands for TM polarization. 


\section{Appendix B}

\section{MATLAB Code}

The code used for measuring the TO coefficients from the spectral shifts of the two waveguides of Fig. 5.15 over a $30 \mathrm{~nm}$ spectral range $(1540-1570 \mathrm{~nm})$ is shown here. The code will be broken down into subsections in order to simplify the analysis. The texts that are in green are comments that will help the reader understand. Refer to section 5.2.4 for further details.

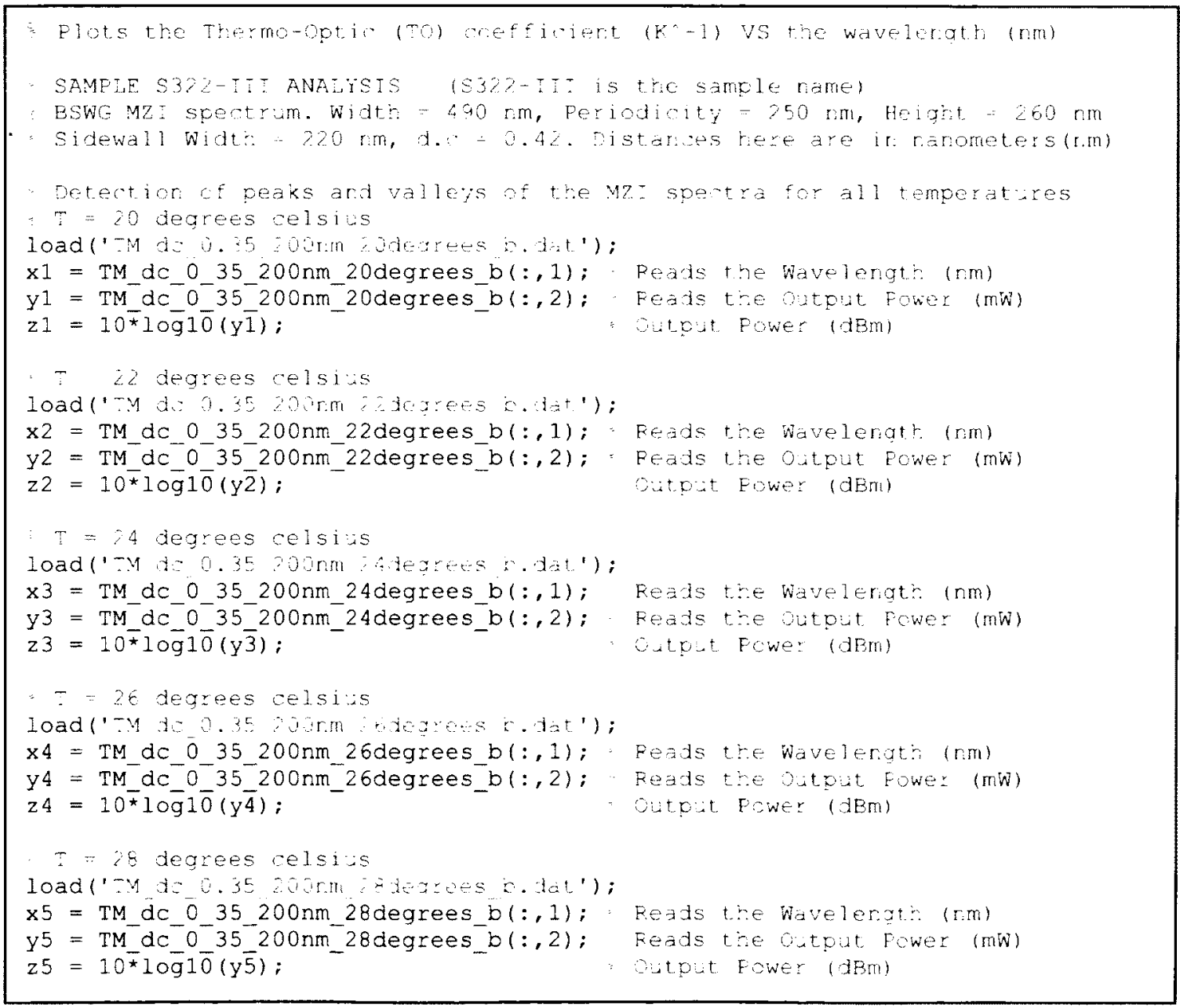


The code that is shown above loads the necessary data files (dat). These contain data points that define the MZI transmission curve for various temperatures. The MZI here comprises BSWGs with $\mathrm{W}_{1}=490 \mathrm{~nm}, \mathrm{~W}_{2}=220 \mathrm{~nm}, \mathrm{H}=260 \mathrm{~nm}, \Lambda=250 \mathrm{~nm}$, and DC $=42 \%$. The guided light is TM polarized.

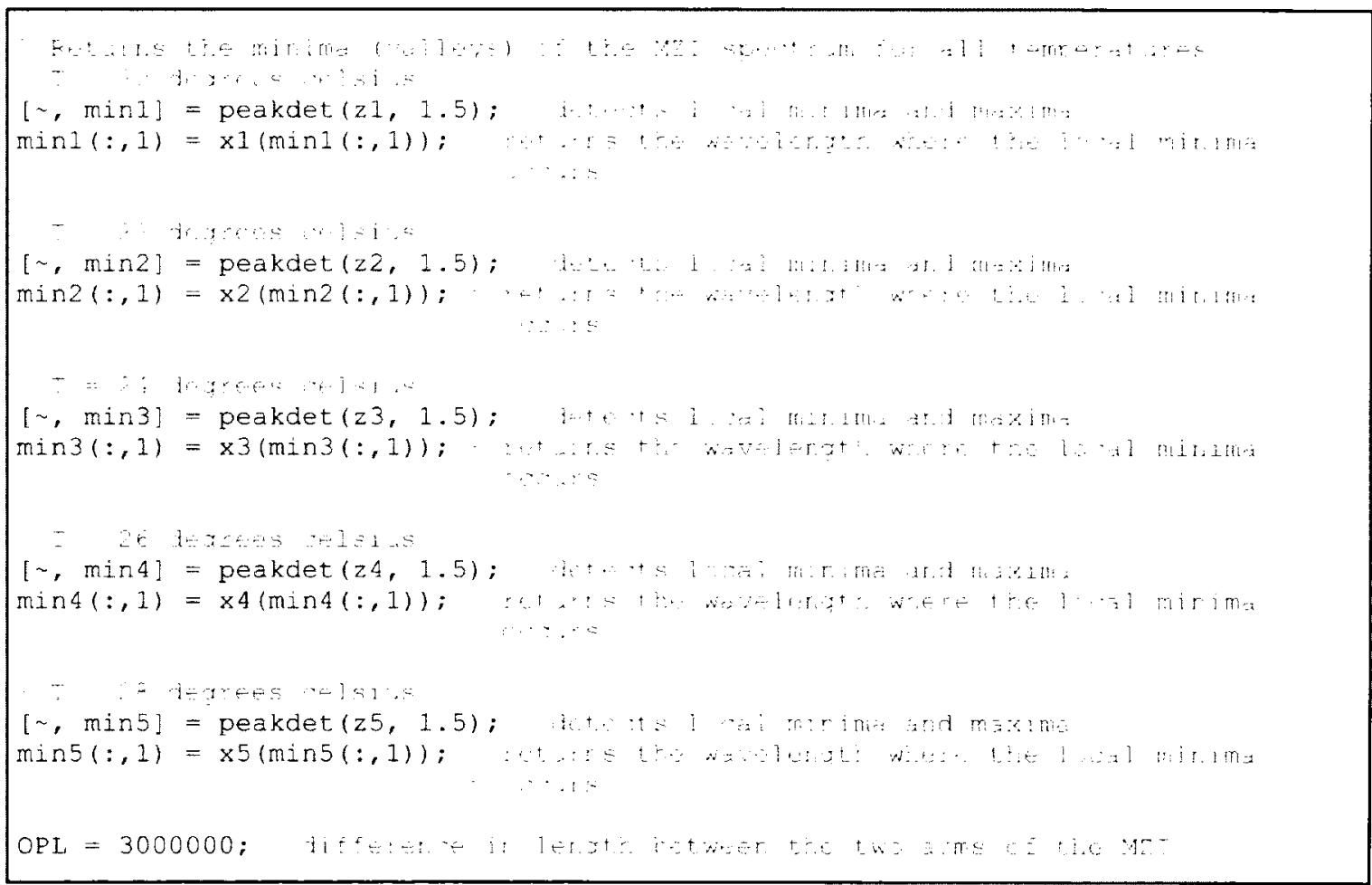

Here, the "min" vectors are generated. They contain the minima (valley) of the MZI curve at the specific temperatures. The optical path length difference (OPL), i.e. the geometrical imbalance, is defined $(\Delta \mathrm{L}=3 \mathrm{~mm})$. Note that the distances in the script are in nanometers. The geometrical imbalance is of importance when measuring the thermooptic coefficient (see equation 5.3). 


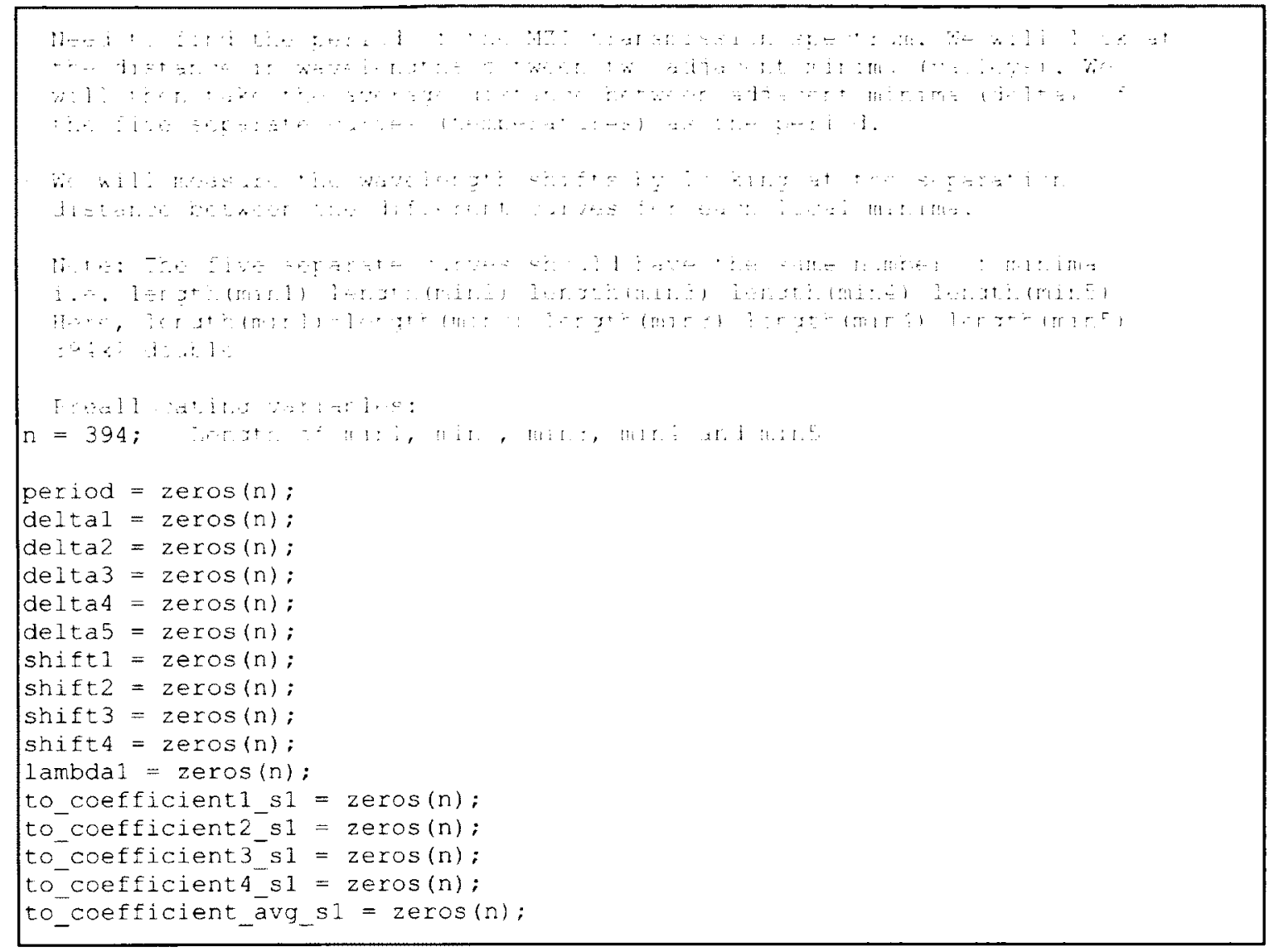

Here, the variables such as the "delta" vectors, i.e. the distance between two successive minima of the MZI of the same temperature, the "shift" vectors, i.e. the spectral shift between the two closest curves in terms of temperature, and the "to_coefficient" vectors, i.e. the resultant TO coefficient that only regards the two closest curves, are preallocated and defined. The "period", "lambdal", and "to_coefficient_avg_s1" are averaging variables. The "period" vector averages out the distance between two successive minima of each curve, the "lambdal" vector averages out the location of the minima of the curves, and "to_coefficient_avg_sl" averages out the TO coefficients found from the two 
successive curves. For further details on these vectors, please refer to the next subsection of the MATLAB script.

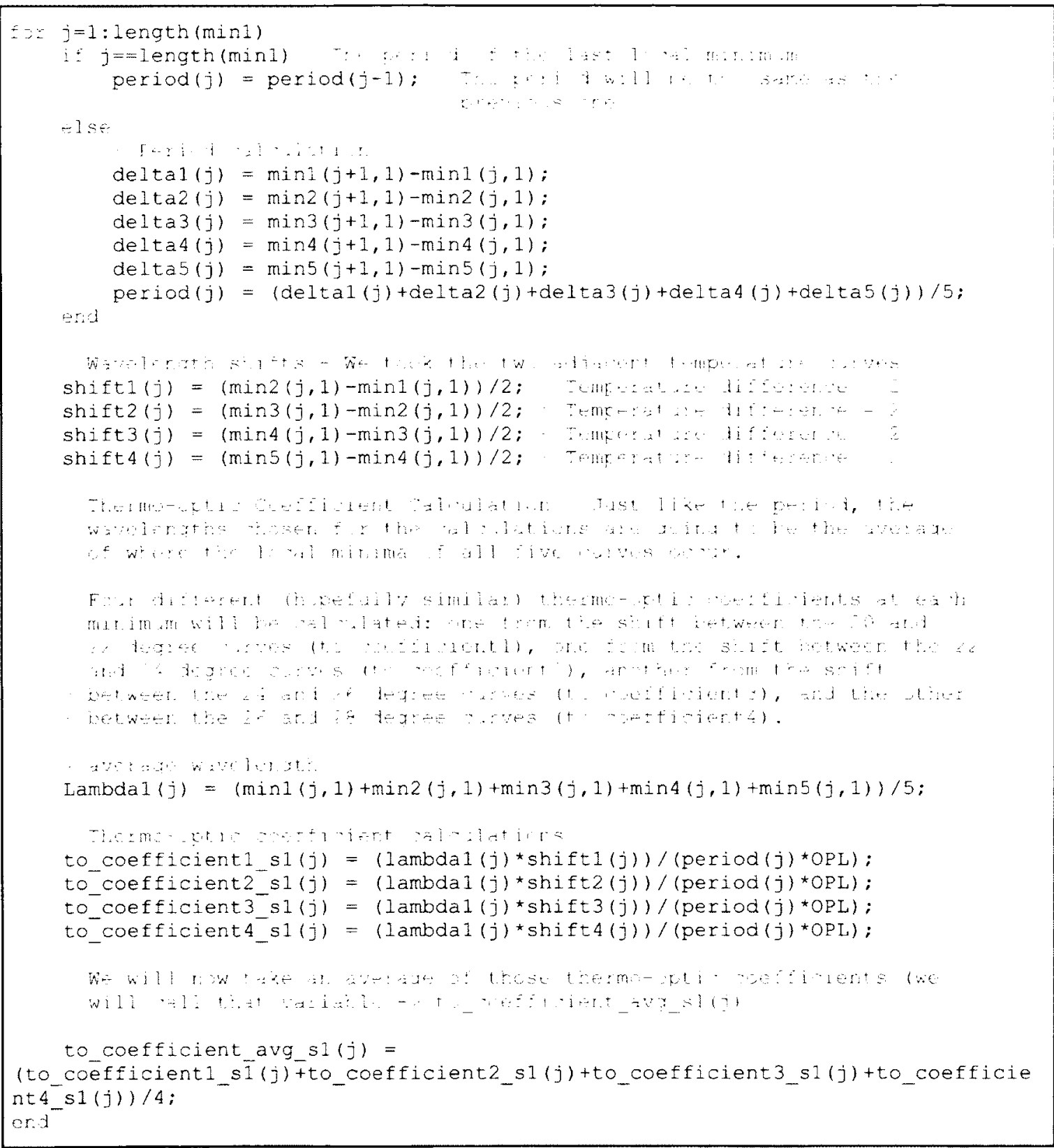

The calculations are explained in the script above. From there, we obtain a vector "to_coefficient_avg_sl" where each element is the averaged value of the BSWG 
effective TO coefficient at a minimum. The location of that minimum corresponds to an element of the vector "lambdal".

We have repeated these steps for each MZI comprising the BSWG structures of interest. Here, we have compared the results obtained from the script above with the ones from the script below. The unbalanced arms of the MZI described below comprise PW waveguides as oppose to BSWGs. The final results which compare both are shown in Fig. 5.16. As you can see, the script is very similar to the one above.

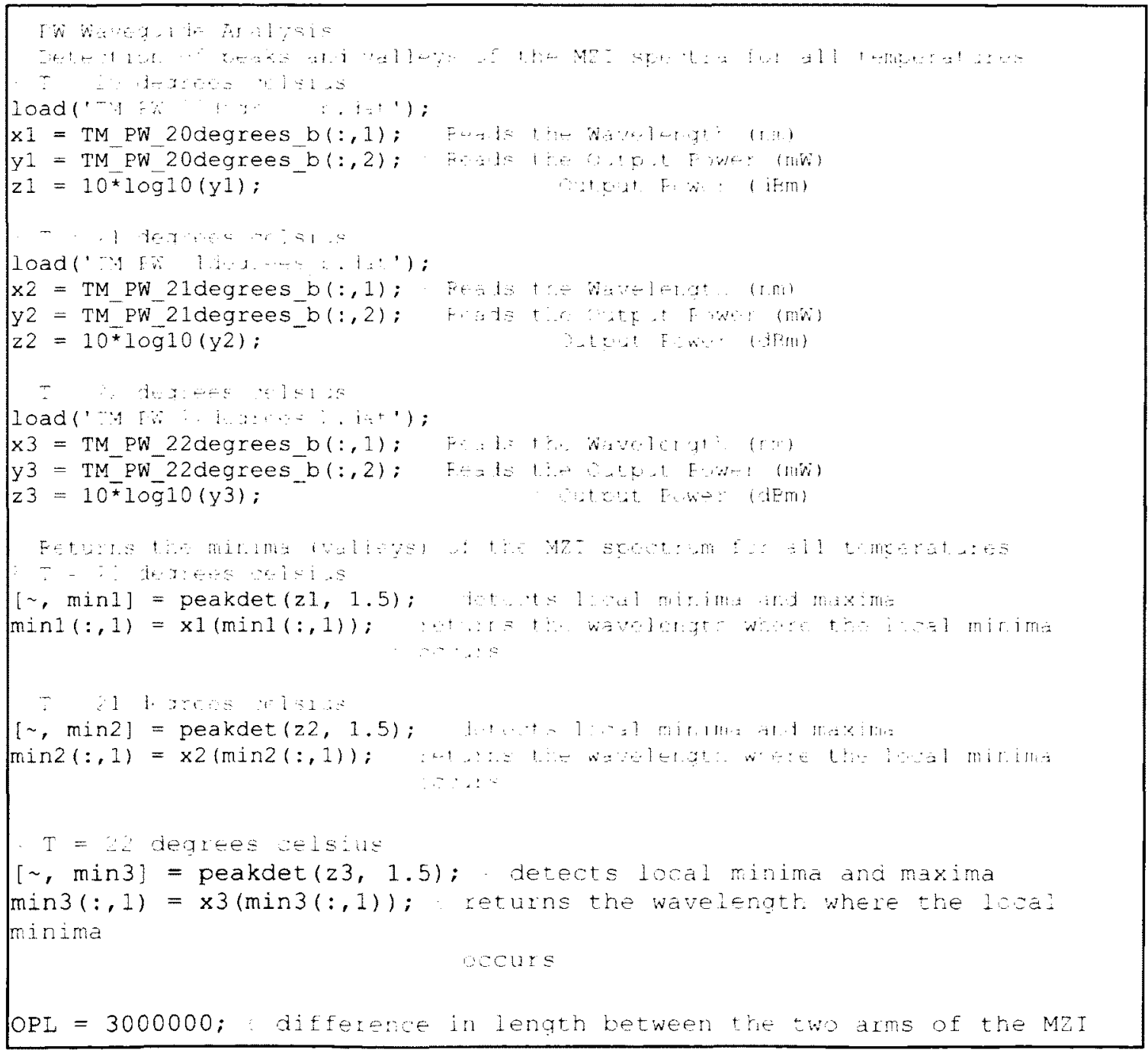














\section{References}

[1] Koehl, S., Liu, A., Paniccia, M., "Integrated Silicon Photonics: Harnessing the Data Explosion", Optics and Photonics News 22 (3) 25-29 (2011)

[2] Schmid, J.H., Ibrahim, M., Cheben, P., Lapointe, J., Janz, S., Bock, P.J., Densmore, A., Lamontagne, B., Ma, R., Ye, W.N., and Xu, D.-X., "Temperatureindependent silicon subwavelength grating waveguides", Optics Lett. 36 (11) 2110-2112 (2011)

[3] Kokubun, Y., Funato, N., Takizawa, M., "Athermal waveguides for temperatureindependent lightwave devices”, IEEE Photonics Technology Letters 5 (11) 1297-1300 (1993)

[4] Kokubun Y., "Athermal waveguides and temperature insensitive lightwave devices", Technical Digest, CLEO/Pacific Rim '99, vol. 4, 1143-1144 (1999)

[5] Kokubun, Y., Takizawa, M., Taga, S., "Three-dimensional athermal waveguides for temperature independent lightwave devices", Electronics Letters 30 (15) 1223-1224 (1994)

[6] Taga, S., Tanaka, H., Kokubun, Y., "Three-dimensional athermal waveguide at $1.3 \mu \mathrm{m}$ wavelength for temperature independent lightwave devices', Optical Review 3 (6B) $478-480$ (1996)

[7] Kokubun, Y., Yoneda, S., Tanaka, H., "Temperature-independent narrow-band filter by athermal waveguide", European Conference on Optical Communication (ECOC), vol. 3, 3143-3146 (1996)

[8] Kokubun, Y., Yoneda, S., Matsuura, S., "Temperature-independent optical filter at $1.55 \mu \mathrm{m}$ wavelength using a silica-based athermal waveguide", Electronics Letters 34 (4) $367-369$ (1998)

[9] Bosc, D., Hurault, N., "Numerical design optimization of ridge waveguide for athermal integrated Bragg grating”, IEEE Photonics Technology Letters 10 (12), 17511753 (1998)

[10] Inoue, Y., Kaneko, A., Hanawa, F., Takahashi, H., Hattori, K., Sumida, S., "Athermal silica-based arrayed-waveguide grating multiplexer", Electronics Letters 33 (23) 1945-1947 (1997)

[11] Kaneko, A., Kamei, S., Inoue, Y., Takahashi, H., Sugita, A., "Athermal silicabased arrayed-waveguide grating (AWG) multiplexers with new low loss groove design", Optical Fiber Communication Conference, vol.1, 204-206 (1999) 
[12] Kamei, S., Inoue, Y., Shibata, T., Kaneko, A., "1.5\%- $\Delta$ athermal arrayedwaveguide grating multi/demultiplexer with very low loss groove design", Journal of Lightwave Technology 27 (17) 3790-3799 (2009)

[13] Saida, T., Kaneko, A., Goh, T., Okuno, M., Himeno, A., Takigushi, K., Okamoto, K., "Athermal silica-based optical add/drop multiplexer consisting of arrayed waveguide gratings and double gate thermo-optical switches", Electronics letters 36 (6) 528-529 (2000)

[14] Takagi, A., Kaneko, A., Ishii, M., Itoh, M., Sugita, A., "Low-loss and flat passband $1 \times 8$ arrayed-waveguide grating multi/demultiplexers with athermal spectrum for metropolitan area networks", Optical Fiber Communication Conference - Trends in optics and Photonics 37, 136-138 (2000)

[15] Kamei, S., Kohtoku, M., Shibata, T., Kitoh, T., "Athermal Mach-Zehnder interferometer-synchronized arrayed waveguide grating multi/demultiplexer with low loss and wide passband", Electronics Letters 44 (3) 201-202 (2008)

[16] Kamei, S., Kitoh, T., Itoh, M., Shibata, T., Kohtoku, M., "50-GHz-spacing athermal Mach-Zehnder interferometer-synchronized arrayed-waveguide grating with improved temperature insensitivity", IEEE Photonics Technology Letters 21 (17) 12051207 (2009)

[17] Maru, K., Ohkawa, M., Nounen, H., Takasugi, S., Kashimura, S., Okano, H., Uetsuka, H., "Athermal and center wavelength adjustable arrayed-waveguide grating" Optical Fiber Communication Conference - Trends in Optics and Photonics 37, 130-132 (2000)

[18] Maru, K., Matsui, K., Ishikawa, H., Abe, Y., Kashimura, S., Himi, S., Uetsuka, $H$. ., "Super-high- $\Delta$ athermal arrayed waveguide grating with resin-filled trenches in slab region", Electronics Letters 40 (6) 374-375 (2004)

[19] Maru, K., Abe, Y., Uetsuka, H., "Demonstration of compact and low-loss athermal arrayed-waveguide grating module based on $2.5 \%-\Delta$ silica-based waveguides", Japanese Journal of Applied Physics 47 (10) 7903-7908 (2008)

[20] Itoh, M., Kamei, S., Ishii, M., Shibata, T., Tamura, M., Inoue, Y., "Ultra small $100 \mathrm{GHz} 40 \mathrm{ch}$ athermal AWG module using $2.5 \%-\Delta$ silica-based waveguides", European Conference on Optical Communication (ECOC), vol. 1, $91-92$ (2008)

[21] Kamei, S., Shibata, T., Inoue, Y., "Compensation for second-order temperature dependence in athermal arrayed-waveguide grating realizing wide temperature range operation", IEEE Photonics Technology Letters 21 (22) 1695-1697 (2009)

[22] Saito, T., Nara, K., Nekado, Y., Hasegawa, J., Kashihara, K., "100GHz-32ch Athermal AWG with Extremely Low Temperature Dependency of Center Wavelength", 
Conference on Optical Fiber Communication, Technical Digest Series, vol. 86, 57-59 (2003)

[23] Huang, G., Yu, S., Massara, A., Heard, P., Penty, R., White, I., "Novel athermal WDM laser with polymer grating", Proceedings of SPIE - the International Society for Optical Engineering, vol. 5280 I, 189-194 (2003)

[24] Li, De-Lu, Ma, C.-S., Qin, Z.-K., Zhang, H.-M., Zhang, D.-M., Liu, S.-Y., "Design of athermal arrayed waveguide grating using silica/polymer hybrid materials", Optica Applicata 37 (3) 305-312 (2007)

[25] Iwamoto, K., Ito, J., Tsuda, H., "An athermal delay interference circuit using trenches filled with low-refractive index material", IEICE Electronics Express 6 (24) 1769-1773 (2009)

[26] Nasu, Y., Sakamaki, Y., Hattori, K., Kamei, S., Hashimoto, T., Saida, T., Takahashi, H., Inoue, Y., "Compact and athermal DQPSK demodulator with silica-based planar lightwave circuit" IEICE Transactions on Electronics E93-C (7) 1191-1198 (2010)

[27] Ooba, N., Hibino, Y., Inoue, Y., Sugita, A., "Athermal silica-based arrayedwaveguide grating multiplexer using bimetal plate temperature compensator", Electronics Letters 36 (21) 1800-1801 (2000)

[28] Hasegawa, J., Nara, K., "Ultra-low-loss athermal AWG module with a large number of channels", Furukawa Review (26) 1-5 (2004)

[29] Hasegawa, J., Nara, K., "Low-loss athermal AWG module with high reliability", Electronics and Communications in Japan, Part II: Electronics 89 (3) 26-33 (2006)

[30] Huang, H., Liu, W., Huang, D., Yu, K., "Analytical solutions for stress compensated athermal arrayed waveguide gratings", SPIE Proceedings, vol. 6781 (2007)

[31] Tae, H. K., Byung, G. Y., Hyung, J. L., Tae, H. R., "Athermal AWG multiplexer/demultiplexer for E/C-band WDM-PON application", 2007 Asia Optical Fiber Communication and Optoelectronic Exposition and Conference, AOE, 330-332 (2007)

[32] Nishii, J., Kintaka, K., Nishiyama, H., Takahashi, M., "Photosensitive and athermal glasses for optical channel waveguides", Journal of Non-Crystalline Solids, vol. 326-327, 464-471 (2003)

[33] Hirota, H., Itoh, M., Oguma, M., Hibino, Y., "Athermal arrayed-waveguide grating multi/demultiplexers composed of $\mathrm{TiO}_{2}-\mathrm{SiO}_{2}$ waveguides on $\mathrm{Si}$ ", IEEE Photonics Technology Letters 17 (2) 375-377 (2005) 
[34] Choudhury, B. D., Pal, S., Singh, B. R., "Optimal design of silica-based temperature-insensitive long-period waveguide gratings for realization of athermal refractive-index sensor", Sensors and Actuators, A: physical 141 (2) 328-333 (2008)

[35] Terui, H., Shibazaki, T., Ebisawa, F., Okuno, M., Jinnouchi, Y., Kamei, S., "Reduction of second-order temperature dependence of athermal AWG with resin-filled groove by pressure control", IEEE Photonics Technology Letters 21 (19) 1426-1428 (2009)

[36] Zhou, T., Ma, W., "A novel fabrication approach for an athermal arrayedwaveguide grating", Journal of Semiconductors 31 (1) 3 pp. (2010)

[37] Lin, C.-T., "Athermal metal-free planar waveguide concave grating demultiplexer", Optics Communications 284 (1) 195-200 (2011)

[38] Wang, X., Xiao, S., Zheng, W., Wang, F., Hao, Y., Jiang, X., Wang, M., Yang, J., "Athermal silicon arrayed waveguide grating with polymer-filled slot structure" Group IV Photonics Conference, p.253-255 (2008)

[39] Wang, X., Xiao, S., Zheng, W., Wang, F., Hao, Y., Jiang, X., Wang, M., Yang, J., "Slot-based athermal silicon arrayed-waveguide grating (AWG)" SPIE Proceedings, vol. 7134,8 pp. (2008)

[40] Ye, W. N., Michel, J., Kimerling, L. C., Eldada, L., "Polymer-cladded athermal high-index-contrast waveguides", SPIE Proceeding, vol. 6897 (2008)

[41] Raghunathan, V., Ye, W.N., Hu J., Izuhara, T., Michel, J., Kimerling, L., "Athermal operation of Silicon waveguides: spectral, second order and footprint dependencies", Optics express 18 (17) 17631-17639 (2010)

[42] Teng, J., Dumon, P., Bogaerts, W., Zhang, H., Jian, X., Han, X., Zhao, M., Morthier, G., Baets, R., "Athermal silicon-on-insulator ring resonators by overlaying a polymer cladding on narrowed waveguides", Optics Express 17 (17) 14627-14633 (2009)

[43] Teng, J., Dumon, P., Bogaerts, W., Zhang, H., Jian, X., Zhao, M., Morthier, G., Baets, R., "Athermal SOI ring resonators by overlaying a polymer cladding on narrowed waveguides", IEEE Group IV Photonics Proceeding, 77-79 (2009)

[44] Zhou, L., Kashiwagi, K., Okamoto, K., Scott, R. P., Fontaine, N. K., Ding, D., Akella, V., Yoo, S. J. B., "Towards athermal optically-interconnected computing system using slotted silicon microring resonators and RF-photonic comb generation", Applied Physics A: Materials Science and Processing 95 (4) 1101-1109 (2009)

[45] Milosevic, M. M., Mashanovich, G.Z., Gardes, F.Y., Hu, Y., Knights, A. P., Tarr, N. G., Reed, G. T., "Athermal and low loss ridge silicon waveguides", SPIE Proceeding, vol. $7606(7 \mathrm{pp}).(2010)$ 
[46] Wang, L., Bogaerts, W., Dumon, P., Selvaraja, S. K., Morthier, G., Teng, J., Han, X., Jian, X., Zhao, M., Baets, R., "Athermal AWGs in SOI by overlaying a polymer cladding on narrowed arrayed waveguides", Optical Fiber Communication (OFC) Conference, 3 pp. (2011)

[47] Keil, N., Yao, H. H., Zawadzki, C., Bauer, J., Bauer, M., dreyer, C., Schneider, J., "Athermal all-polymer arrayed-waveguide grating multiplexer", Electronics Letters 37 (9) $579-580(2001)$

[48] Yao, H. H., Zawadzki, C., Keil, N., "Athermal all-polymer arrayed waveguide grating multiplexer", Optical Fiber Communications Conference, vol.1, 12-14 (2002)

[49] Han, X., Zhao, M., Zhang, J., Wang, L., Teng, J., Wang, J., Jian, X., "Design of athermal all-polymer waveguide microring resonator", SPIE proceedings, vol. 7630,8 pp. (2009)

[50] P. Cheben, P. J. Bock, J. H. Schmid, J. Lapointe, S. Janz,D.-X. Xu, A. Densmore, A. Delâge, B. Lamontagne, and T. J. Hall, "Refractive index engineering with subwavelength gratings for efficient microphotonic couplers and planar waveguide multiplexers", Opt. Lett. 35 (15) 2526-2528 (2010)

[51] P.J. Bock, P. Cheben, J.H. Schmid, J. Lapointe, A. Delâge, S. Janz, G.C. Aers, D.$\mathrm{X}$. Xu, A. Densmore, and T.J. Hall, "Subwavelength grating periodic structures in silicon-on-insulator: a new type of microphotonic waveguide", Opt. Express 18 (19) 20251-20262 (2010)

[52] F. P. Payne, and J. P. R. Lacey, "A theoretical analysis of scattering loss from planar optical waveguides," Opt. Quantum Electron. 26(10), 977-986 (1994)

[53] F. Grillot, L. Vivien, S. Laval, D. Pascal, and E. Cassan, "Size influence on the propagation loss induced by sidewall roughness in ultra small SOI waveguides," IEEE Photon. Technol. Lett. 16(7), 1661-1663 (2004)

[54] J.H. Schmid, P. Cheben, S. Janz, J. Lapointe, E. Post, A. Delâge, A. Densmore, B. Lamontagne, P. Waldron, and D.-X. Xu, "Subwavelength Grating Structures in Siliconon-Insulator Waveguides", Advances in Opt. Technol. Vol 2008, 8 pages (2008)

[55] J. D. Joannopoulos, R. D. Meade, and J. N. Winn, "Photonic Crystals: Molding the Flow of Light", 184 pages (1995)

[56] J. H. Schmid, P. J. Bock, P. Cheben, W. Sinclair, J. Garcia, S. Janz, J. Lapointe, G. C. Aers, D. Poitras, Y. Li, G. Lopinski, A. Delâge, A. Densmore, B. Lamontagne, R. $\mathrm{Ma}$, and D.-X. Xu, "Applications of subwavelength grating structures in silicon-on insulator waveguides", SPIE proceedings vol. 7606 (2010) 
[57] Z. Zhang, P. Zhao, P. Lin, and F. Sun, "Thermo-optic coefficients of polymers for optical waveguide applications", ScienceDirect vol. 47, 4893-4896 (2006)

[58] S. G. Johnson, "The MIT Photonic-Bands Manual", Massachusetts Institute of Technology, 64 pages, http://ab-initio.mit.edu/mpb/doc/mpb.pdf (2002)

[59] Ghosh, G., "Model for the thermo-optic coefficients of some standard optical glasses", J. Non-Crystalline Solids, 189, 191-196 (1995)

[60] P. Cheben, J. H. Schmid, P. J. Bock, J. Lapointe, S. Janz, D.-X. Xu, A. Delâge, M. Vachon, R. Halir, A. Ortega-Monux, C. Alonso Ramos, I. Molina-Fernandez, J.-M. Fédéli, M. Ibrahim, W. N. Ye, A. V. Velasco, M. L. Calvo, and I. Glesk, "Refractive Index Engineering with Sub-Wavelength Gratings in Silicon Waveguides", Advanced Photonics Congress IPRSN, OSA, (2012)

[61] J. H. Schmid, W. Sinclair, J. Garcia, S. Janz, J. Lapointe, D. Poitras, Y. Li, T. Mischki, G. Lopinski, P. Cheben, A. Delâge, A. Densmore, P. Waldron, and D.-X. Xu, "Silicon-on-insulator guided mode resonant grating for evanescent field molecular sensing", Optics Express 17 (20) 18371-18380 (2009) 